\title{
Improved Synthesis of $\beta$-Aminoboronate Esters via Copper-Catalyzed Diastereo- and Enantioselective Addition of 1,1-Diborylalkanes to Acyclic Arylaldimines
}

\author{
Jeongho Kim, ${ }^{\dagger}$ Chiwon Hwang, ${ }^{\dagger}$ Youngmin Kim, and Seung Hwan Cho* \\ Department of Chemistry, Pohang University of Science and Technology (POSTECH), \\ Pohang, 37673, Rep. of Korea
}

\section{Table of Contents}

1. General experimental details

2. General procedure for the optimization study

3. General procedure for the diastereo- and enantioselective addition of 1,1diborylalkanes to $N, N$-dimethyl- $N$ '-(phenylmethylene)sulfamide derivatives

4. General procedure for synthetic applications

Appendix I. Spectral Copies of ${ }^{1} \mathrm{H},{ }^{11} \mathrm{~B},{ }^{13} \mathrm{C}$ and ${ }^{19} \mathrm{~F}$ NMR Data Obtained in this Study

Appendix II. Crystallographic Data of 3a 


\section{General experimental details}

All air-sensitive manipulations were conducted under an inert atmosphere in a nitrogenfilled glovebox or by standard Schlenk techniques. Glassware was dried at $120{ }^{\circ} \mathrm{C}$ for at least $3 \mathrm{~h}$ prior to use. Unless otherwise noted, all of chemicals were purchased from Alfa Aesar, Sigma-Aldrich, TCI and used without further purification. Toluene and 1,4-dioxane were purchased from Aldrich and vigorously sparged with $\mathrm{N}_{2}$ prior to use. $\mathrm{CuBr}(99 \%)$ and $\mathrm{LiO} t \mathrm{Bu}(99 \%)$ were purchased from Alfa Aesar and used as received. Chiral ligands $\mathbf{L} \mathbf{1}^{1}$ and $\mathbf{L 2}-\mathbf{L 3}^{2}$ were prepared according to literature procedures. 1,1-Diborylalkanes were synthesized according to literature procedures. ${ }^{3-6} N, N$-Dimethylsulfamoyl protected acyclic arylaldimines 1 were synthesized according to literature procedures. ${ }^{7}$ Enantiomeric excesses (\% ee) were determined either by chiral HPLC analysis using Agilent 1200 Series or Shimadzu LC-20A chromatographs using a mixture of HPLC-grade hexane and isopropanol as eluent. Specific columns and analytic methods were provided in the experimental details for individual compounds; the wavelengths of light used for chiral analyses are provided with the associated chromatograms. Optical rotations measured on Perkin Elmer 241 and JASCO P1030-polarimeters equipped with sodium vapor laps at $589 \mathrm{~nm}$ sample concentrations. Analytical thin layer chromatography (TLC) was performed on Merck precoated silica gel 60 F254 plates. Visualization of TLC was achieved by the use of UV light $(254 \mathrm{~nm})$, or treatment with $\mathrm{KMnO}_{4}$ stain followed by heating. Column chromatography was performed on silica gel (400-630 mesh) using a proper eluent system. NMR spectrums were acquired on $300 \mathrm{MHz}, 500 \mathrm{MHz}$ and $600 \mathrm{MHz}$ Bruker instruments at the POSTECH NMR facility. Chemical shifts are reported in ppm relative to a residual solvent peak $\left(\mathrm{CDCl}_{3}=\right.$ $7.26 \mathrm{ppm}$ for ${ }^{1} \mathrm{H}$ and $77.16 \mathrm{ppm}$ for ${ }^{13} \mathrm{C}$ ). For all spectra, multiplicities are given as: $\mathrm{s}$ (singlet), $\mathrm{d}$ (doublet), $\mathrm{t}$ (triplet), $\mathrm{q}$ (quartet), $\mathrm{m}$ (multiplet). The resonances for the carbon atoms attached to boron were not observed due to the boron quadrupole. High-resolution mass spectra (HRMS) were obtained from the KBSI (Korea Basic Science Institute) in Daegu by electron impact (EI) ionization technique (magnetic sector-electric sector double focusing mass analyzer). 


\section{General procedure for the optimization study}

In a nitrogen-filled glove-box, $\mathrm{CuBr}(1.4 \mathrm{mg}, 0.010 \mathrm{mmol}, 5.0 \mathrm{~mol} \%)$, ligand $(0.020 \mathrm{mmol}$, $10 \mathrm{~mol} \%$ ), LiO $t \mathrm{Bu}$ (32 mg, $0.40 \mathrm{mmol}$ ), 1,1-diboryl-3-phenylpropane (112 mg, $0.30 \mathrm{mmol}$ ), and solvent $(0.40 \mathrm{~mL})$ were added to a $4.0 \mathrm{~mL}$ dram vial with a magnetic stir bar. The vial was sealed with a PTFE/silicone-lined septum cap and stirred at room temperature for $1 \mathrm{~h}$. To this solution, $N$ - protected acyclic arylaldimine $(1,0.20 \mathrm{mmol})$ was added. The vial was removed from the glove-box, and stirred at $50{ }^{\circ} \mathrm{C}$ for $24 \mathrm{~h}$. The reaction mixture was poured into a separatory funnel and saturated aqueous $\mathrm{NH}_{4} \mathrm{Cl}$ solution $(10 \mathrm{~mL})$ was added. The aqueous phase was extracted with $\mathrm{CH}_{2} \mathrm{Cl}_{2}(10 \mathrm{~mL} \times 3)$ and the combined organic layers were dried over $\mathrm{MgSO}_{4}$, filtered, and concentrated under reduced pressure. The yield and diastereoselectivity of the obtained $\beta$-aminoboronate ester was determined by ${ }^{1} \mathrm{H}-\mathrm{NMR}$ using 1,1,2,2-tetrachloroethane as an internal standard. The crude mixture was purified by silica gel column chromatography to give the desired $\beta$-aminoboronate ester. The enantiomeric excesses were then determined by HPLC.

Table S1. Evaluation of reaction conditions for the 1,2-addition of 2a to acyclic imine

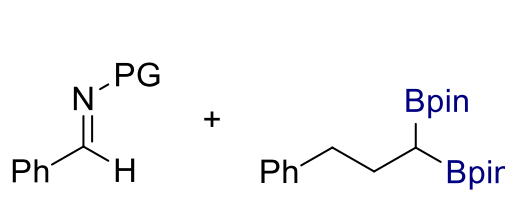

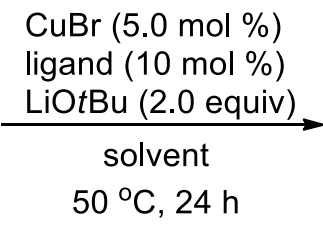

$2 a$<smiles>O=[PH](NC(c1ccccc1)[C@@H](CCc1ccccc1)c1ccccc1)c1ccccc1</smiles>

3

\section{Imines}

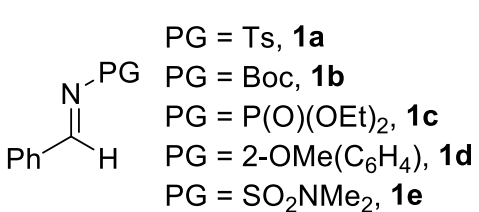

\section{Ligands}

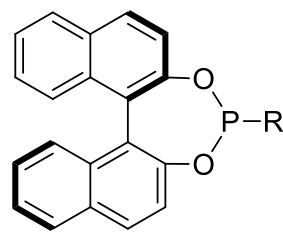

$\mathrm{L1}, \mathrm{R}=\mathrm{NMe}_{2}$

L2, $\mathrm{R}=\mathrm{NEt}_{2}$

L3, $\mathrm{R}=\mathrm{N}\left(\mathrm{CH}_{2} \mathrm{CH}_{2}\right)_{2} \mathrm{O}$

\begin{tabular}{lllllll}
\hline Entry & Imine & L & Solvent & Yield (\%) & d.r. & ee $(\%)$ \\
\hline 1 & 1a & L1 & THF & 59 & $5: 1$ & 98 \\
2 & 1b & L1 & THF & $<1$ & n.d. & n.d. \\
3 & 1c & L1 & THF & $<1$ & n.d. & n.d. \\
4 & 1d & L1 & THF & $<1$ & n.d. & n.d. \\
5 & 1e & L1 & THF & 33 & $13: 1$ & 97 \\
6 & 1e & L1 & 1,4-Dioxane & 56 & $13: 1$ & 97 \\
7 & 1e & L1 & Toluene & 6 & n.d. & n.d. \\
8 & 1e & L1 & 1,4-Dioxane/toluene $(1: 1)$ & 84 & $13: 1$ & 97 \\
9 & 1e & L2 & 1,4-Dioxane/toluene $(1: 1)$ & 71 & $13: 1$ & 96 \\
10 & 1e & L3 & 1,4-Dioxane/toluene $(1: 1)$ & 73 & $12: 1$ & 96 \\
11 & 1a & L1 & 1,4-Dioxane/toluene $(1: 1)$ & trace & n.d. & n.d. \\
\hline
\end{tabular}

${ }^{a}$ Runs at room temperature. n.d. $=$ not determined. Pin $=$ pinacolato. $\mathrm{Ts}=p$-toluenesulfonyl. Boc $=$ tert-butyloxycarbonyl. 


\section{General procedure for diastereo- and enantioselective addition of 1,1-diborylalkanes to $N, N$-dimethyl- $N^{\prime}$-(phenylmethylene)sulfamide derivatives}

In a nitrogen-filled glove-box, $\mathrm{CuBr}(1.4 \mathrm{mg}, 0.010 \mathrm{mmol}, 5.0 \mathrm{~mol} \%), \mathbf{L 1}$ (7.2 mg, 0.020 mmol, $10 \mathrm{~mol} \%)$, LiOt $\mathrm{Bu}$ (32 mg, $0.40 \mathrm{mmol}), 1,1$-diborylalkane $(0.30$ or $0.40 \mathrm{mmol})$, and 1,4-dioxane/toluene $(0.40 \mathrm{~mL}, \mathrm{v} / \mathrm{v} 1: 1)$ were added to a $4.0 \mathrm{~mL}$ dram vial with a magnetic stir bar. The vial was sealed with a PTFE/silicone-lined septum cap and stirred at room temperature for $1 \mathrm{~h}$. To this solution, the corresponding $N, N$-dimethylsulfamoyl protected acyclic arylaldimine $(0.20 \mathrm{mmol})$ was added. The vial was removed from the glove-box, and stirred at $50{ }^{\circ} \mathrm{C}$ (or at room temperature) for $24 \mathrm{~h}$ ( or $48 \mathrm{~h}$ ). The reaction mixture was poured into a separatory funnel and saturated aqueous $\mathrm{NH}_{4} \mathrm{Cl}$ solution $(10 \mathrm{~mL})$ was added. The aqueous phase was extracted with $\mathrm{CH}_{2} \mathrm{Cl}_{2}(10 \mathrm{~mL} \times 3)$ and the combined organic layers were dried over $\mathrm{MgSO}_{4}$, filtered, and concentrated under reduced pressure. The diastereoselectivity was then determined by ${ }^{1} \mathrm{H}$-NMR using 1,1,2,2-tetrachloroethane as an internal standard. The crude mixture was purified by silica gel column chromatography to give the desired $\beta$-aminoboronate ester. The enantiomeric excesses were then determined by HPLC. The absolute and relative configurations of $\beta$-aminoboronate ester $\mathbf{3 a}$ were assigned as $(S, R)$, as determined by single-crystal $\mathrm{X}$-ray analysis. The absolute and relative configurations of $\mathbf{3 b - 3 o}$ were determined by analogy to $\mathbf{3 a}$. 
<smiles>CNS(=O)(=O)NC(c1ccccc1)C(CCc1ccccc1)c1ccccc1</smiles>

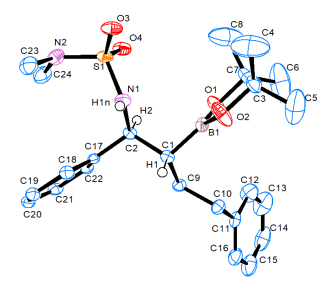

\section{$N, N$-Dimethyl- $N^{\prime}$-[(1S,2R)-1,4-diphenyl-2-(4,4,5,5-tetramethyl-1,3,2-dioxaborolan-2-} yl)butyl]sulfamide (Table 2, 3a): $\mathrm{CuBr}(1.4 \mathrm{mg}, 0.010 \mathrm{mmol}, 5.0 \mathrm{~mol} \%)$, L1 (7.2 mg, $0.020 \mathrm{mmol}, 10 \mathrm{~mol} \%$ ), LiOt $\mathrm{Bu}$ (32 mg, $0.40 \mathrm{mmol}$ ), 1,1-diboryl-3-phenylpropane (112 mg, $0.30 \mathrm{mmol}$ ), $N, N$-dimethyl- $N$ '-(phenylmethylene)sulfamide (43 $\mathrm{mg}, 0.20 \mathrm{mmol}$ ) and 1,4dioxane/toluene $(0.40 \mathrm{~mL}$, v/v $1: 1)$ at $50{ }^{\circ} \mathrm{C}$ for $24 \mathrm{~h} .{ }^{1} \mathrm{H}-\mathrm{NMR}$ analysis of the crude mixture indicated 13:1 diastereoselectivity. The crude mixture was purified by silica gel column chromatography $\left(\mathrm{Et}_{2} \mathrm{O}: n\right.$-hexane $=1: 2$ to $\left.1: 1\right)$ to give the corresponding $\beta$-aminoboronate ester 3a as a white solid (73 mg, 80\%, 97\% ee); $[\alpha]_{\mathrm{D}}^{22}-3.4\left(c 0.59, \mathrm{CH}_{2} \mathrm{Cl}_{2}\right)$; Major diastereomer; ${ }^{1} \mathrm{H}$ NMR $\left(500 \mathrm{MHz}, \mathrm{CDCl}_{3}\right) \delta 7.32-7.25(\mathrm{~m}, 6 \mathrm{H}), 7.24-7.21(\mathrm{t}, J=7.0 \mathrm{~Hz}$, $1 \mathrm{H}), 7.19-7.16(\mathrm{~m}, 3 \mathrm{H}), 5.55-5.53(\mathrm{~d}, J=8.3 \mathrm{~Hz}, 1 \mathrm{H}), 4.53-4.50(\mathrm{dd}, J=8.1,6.1 \mathrm{~Hz}$, 1H), $2.77-2.66(\mathrm{~m}, 2 \mathrm{H}), 2.48(\mathrm{~s}, 6 \mathrm{H}), 1.89-1.75(\mathrm{~m}, 2 \mathrm{H}), 1.49-1.44$ (dd, $J=14.2,6.6$ $\mathrm{Hz}, 1 \mathrm{H}), 1.26-1.22(\mathrm{~d}, J=20.5 \mathrm{~Hz}, 12 \mathrm{H}) ;{ }^{13} \mathrm{C} \mathrm{NMR}\left(126 \mathrm{MHz}, \mathrm{CDCl}_{3}\right) \delta 143.4,142.0$, 128.6, 128.4 (x 2), 127.3, 126.6, 125.9, 84.0, 59.1, 37.6, 35.1, 30.9, 24.9; ${ }^{11} \mathrm{~B}$ NMR (161 $\left.\mathrm{MHz}, \mathrm{CDCl}_{3}\right) \delta 33.7$; HRMS (EI) $\mathrm{m} / \mathrm{z}$ calcd. for $\mathrm{C}_{24} \mathrm{H}_{35} \mathrm{BN}_{2} \mathrm{O}_{4} \mathrm{~S}[\mathrm{M}]^{+}: 458.2411$, found: 458.2408; CHIRALCEL IB, $n$-hexane : $i$-PrOH $=98: 2,1 \mathrm{~mL} / \mathrm{min}, 25^{\circ} \mathrm{C}, \lambda=220 \mathrm{~nm}$; $\mathrm{t}_{\mathrm{R}} 6.2$ $\min$ (minor), $t_{R} 7.5$ min (major); Analogous mixtures of enantiomers were prepared using $( \pm)-\mathbf{L} \mathbf{1}$ as a ligand under the standard reaction procedure.

[Racemic 3a]

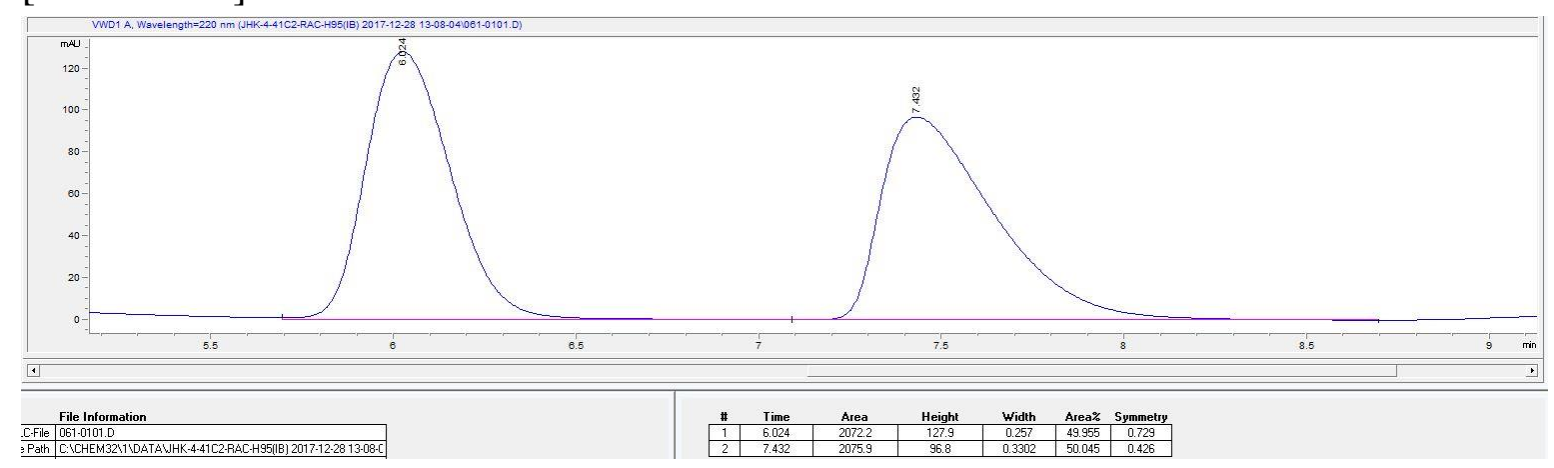

[Enantioenriched 3a]

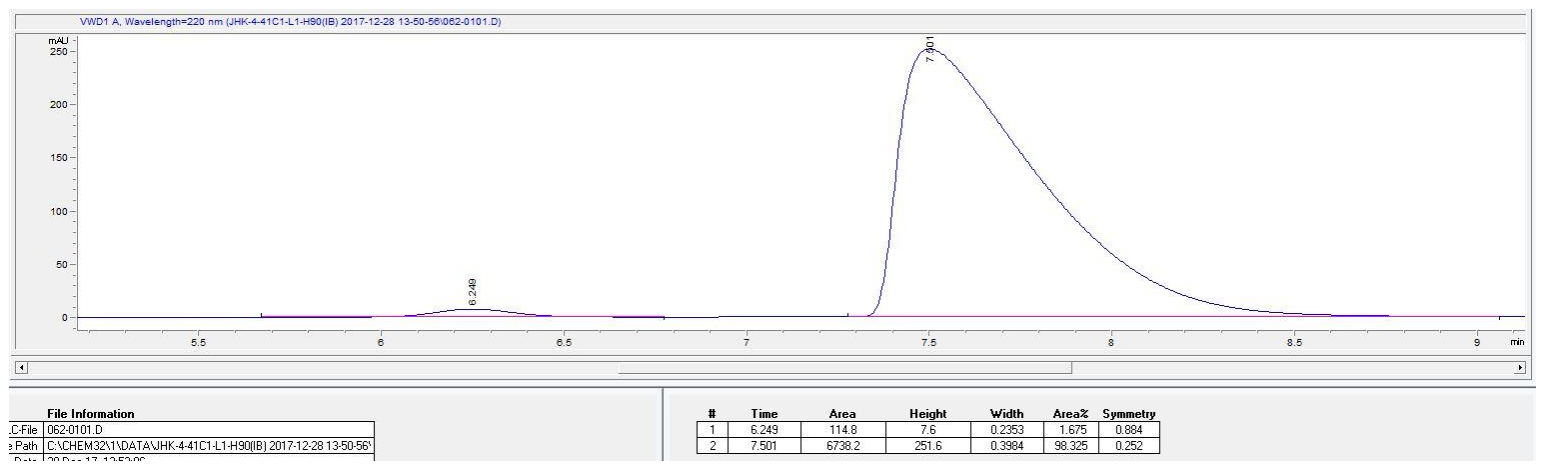


<smiles>CN(C)S(=O)(=O)N[C@H](c1ccc(C(F)(F)F)cc1)[C@@H](CCc1ccccc1)c1ccccc1</smiles>

$N, N$-Dimethyl- $N^{\prime}$-[(1S,2R)-4-phenyl-2-(4,4,5,5-tetramethyl-1,3,2-dioxaborolan-2-yl)-1(4-(trifluoromethyl)phenyl)butyl]sulfamide (Table 2, 3b): $\mathrm{CuBr}(1.4 \mathrm{mg}, 0.010 \mathrm{mmol}$, $5.0 \mathrm{~mol} \%)$, L1 (7.2 mg, $0.020 \mathrm{mmol}, 10 \mathrm{~mol} \%$ ), LiOt $\mathrm{Bu}$ (32 mg, $0.40 \mathrm{mmol}), 1,1$-diboryl3-phenylpropane (112 mg, $0.30 \mathrm{mmol}), \quad N, N$-dimethyl- $N$ '-[(4-(trifluoromethylphenyl) methylene)]sulfamide $(56 \mathrm{mg}, 0.20 \mathrm{mmol})$ and 1,4-dioxane/toluene $(0.40 \mathrm{~mL}, \mathrm{v} / \mathrm{v} 1: 1)$ at 50 ${ }^{\circ} \mathrm{C}$ for $48 \mathrm{~h}$. ${ }^{1} \mathrm{H}-\mathrm{NMR}$ analysis of the crude mixture indicated 15:1 diastereoselectivity. The crude mixture was purified by silica gel column chromatography $\left(\mathrm{Et}_{2} \mathrm{O}: n\right.$-hexane $=1: 2$ to $1: 1)$ to give the corresponding $\beta$-aminoboronate ester $\mathbf{3 b}$ as a colorless gel (67 $\mathrm{mg}, 64 \%$, 99\% ee); $[\alpha]_{\mathrm{D}}^{22}-10.5\left(c 0.63, \mathrm{CH}_{2} \mathrm{Cl}_{2}\right.$ ); Major diastereomer; ${ }^{1} \mathrm{H} \mathrm{NMR}\left(500 \mathrm{MHz}, \mathrm{CDCl}_{3}\right) \delta$ $7.58-7.56(\mathrm{~d}, J=8.1 \mathrm{~Hz}, 2 \mathrm{H}), 7.40-7.38(\mathrm{~d}, J=8.0 \mathrm{~Hz}, 2 \mathrm{H}), 7.30-7.27(\mathrm{t}, J=7.6 \mathrm{~Hz}$, $2 \mathrm{H}), 7.21-7.18(\mathrm{t}, J=6.7 \mathrm{~Hz}, 3 \mathrm{H}), 5.61-5.59(\mathrm{~d}, J=8.2 \mathrm{~Hz}, 1 \mathrm{H}), 4.58-4.56(\mathrm{dd}, J=7.9$, $5.6 \mathrm{~Hz}, 1 \mathrm{H}), 2.79-2.68(\mathrm{~m}, 2 \mathrm{H}), 2.55(\mathrm{~s}, 6 \mathrm{H}), 1.93-1.78(\mathrm{~m}, 2 \mathrm{H}), 1.46-1.42(\mathrm{dd}, J=13.2$, $7.1 \mathrm{~Hz}, 1 \mathrm{H}), 1.25-1.21(\mathrm{~d}, J=18.7 \mathrm{~Hz}, 12 \mathrm{H}) ;{ }^{13} \mathrm{C} \mathrm{NMR}\left(126 \mathrm{MHz}, \mathrm{CDCl}_{3}\right) \delta 147.6,141.7$, $129.6(\mathrm{q}, J=32.6 \mathrm{~Hz}), 128.6,128.5,127.0,126.1,125.4(\mathrm{q}, J=3.7 \mathrm{~Hz}), 124.2(\mathrm{q}, J=272$ $\mathrm{Hz}) 84.2,58.8,37.8,35.0,30.8,25.0,24.9 ;{ }^{19} \mathrm{~F} \mathrm{NMR}\left(471 \mathrm{MHz}, \mathrm{CDCl}_{3}\right) \delta-62.4 ;{ }^{11} \mathrm{~B} \mathrm{NMR}$ $\left(161 \mathrm{MHz}, \mathrm{CDCl}_{3}\right) \delta$ 33.9; HRMS (EI) $\mathrm{m} / \mathrm{z}$ calcd. for $\mathrm{C}_{25} \mathrm{H}_{34} \mathrm{BF}_{3} \mathrm{~N}_{2} \mathrm{O}_{4} \mathrm{~S}[\mathrm{M}]^{+}:$: 26.2284 , found: 526.2283; CHIRALCEL IB, $n$-hexane : $i$-PrOH $=97: 3,1 \mathrm{~mL} / \mathrm{min}, 25^{\circ} \mathrm{C}, \lambda=220$ $\mathrm{nm} ; \mathrm{t}_{\mathrm{R}} 4.4 \mathrm{~min}$ (minor), $\mathrm{t}_{\mathrm{R}} 5.1 \mathrm{~min}$ (major); Analogous mixtures of enantiomers were prepared using $( \pm)-\mathbf{L} \mathbf{1}$ as a ligand under the standard reaction procedure.

[Racemic 3b]

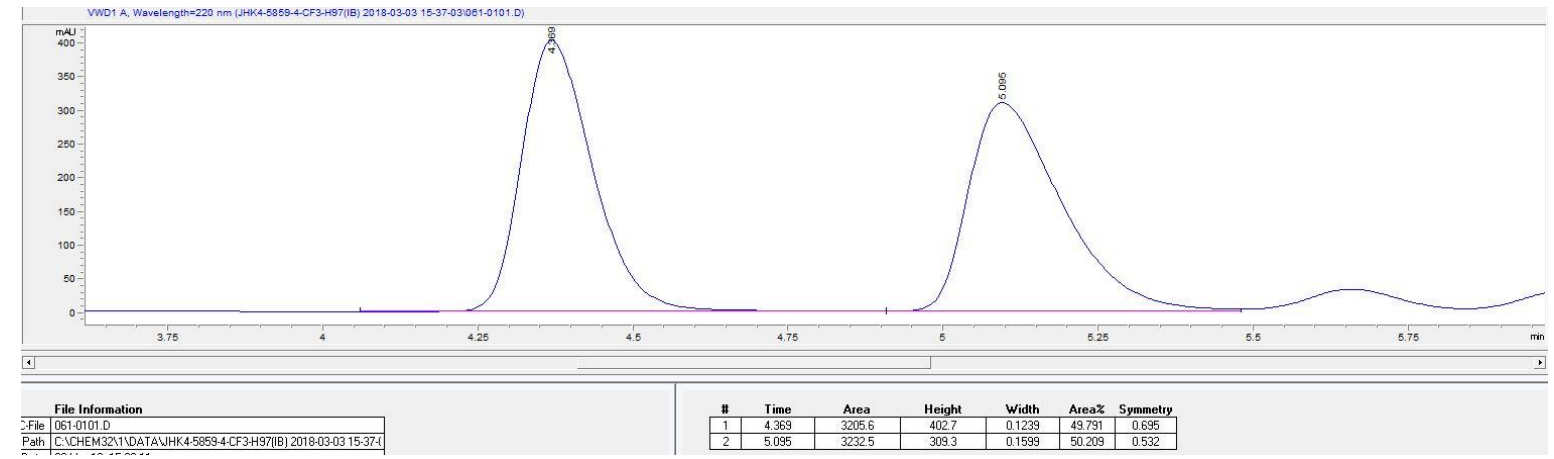

[Enantioenriched 3b]

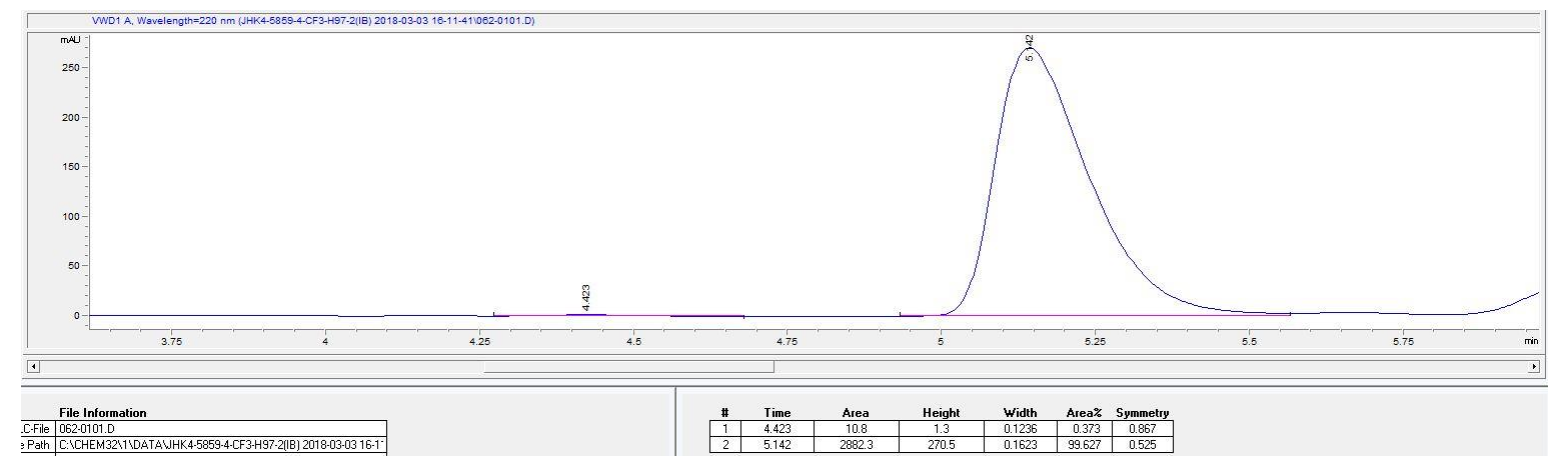


<smiles>CS(=O)(=O)N[C@H](c1ccc(F)cc1)[C@@H](CCc1ccccc1)c1ccccc1</smiles>

\section{$N, N$-Dimethyl- $N^{\prime}$-[(1S,2R)-1-(4-fluorophenyl)-4-phenyl-2-(4,4,5,5-tetramethyl-1,3,2-}

dioxaborolan-2-yl)butyl]sulfamide (Table 2, 3c): $\mathrm{CuBr}(1.4 \mathrm{mg}, 0.010 \mathrm{mmol}, 5.0 \mathrm{~mol} \%$ ), L1 (7.2 mg, $0.020 \mathrm{mmol}, 10 \mathrm{~mol} \%$ ), LiOtBu (32 mg, $0.40 \mathrm{mmol}$ ), 1,1-diboryl-3phenylpropane $(112 \mathrm{mg}, \quad 0.30 \mathrm{mmol}), \quad N, N$-dimethyl- $N^{\prime}-[(4-$ fluorophenyl)methylene $)]$ sulfamide (46 mg, $0.20 \mathrm{mmol})$ and 1,4-dioxane/toluene $(0.40 \mathrm{~mL}, \mathrm{v} / \mathrm{v} 1: 1)$ at $50{ }^{\circ} \mathrm{C}$ for $24 \mathrm{~h}$. ${ }^{1} \mathrm{H}-\mathrm{NMR}$ analysis of the crude mixture indicated 14:1 diastereoselectivity. The crude mixture was purified by silica gel column chromatography $\left(\mathrm{Et}_{2} \mathrm{O}: n\right.$-hexane $=1: 2$ to $\left.1: 1\right)$ to give the corresponding $\beta$-aminoboronate ester $3 \mathbf{c}$ as a white solid (76 $\mathrm{mg}, 80 \%, 99 \%$ ee); $[\alpha]_{\mathrm{D}}^{22}-7.3\left(c\right.$ 0.76, $\left.\mathrm{CH}_{2} \mathrm{Cl}_{2}\right)$; Major diastereomer; ${ }^{1} \mathrm{H} \mathrm{NMR}\left(500 \mathrm{MHz}, \mathrm{CDCl}_{3}\right) \delta 7.32-7.25$ $(\mathrm{m}, 4 \mathrm{H}), 7.22-7.19(\mathrm{t}, J=7.7 \mathrm{~Hz}, 3 \mathrm{H}), 7.03-7.00(\mathrm{t}, J=8.6 \mathrm{~Hz}, 2 \mathrm{H}), 5.52-5.51(\mathrm{~d}, J=$ $8.0 \mathrm{~Hz}, 1 \mathrm{H}), 4.53-4.50(\mathrm{dd}, J=7.7,6.2 \mathrm{~Hz}, 1 \mathrm{H}), 2.77-2.67(\mathrm{~m}, 2 \mathrm{H}), 2.54(\mathrm{~s}, 6 \mathrm{H}), 1.90-$ $1.75(\mathrm{~m}, 2 \mathrm{H}), 1.47-1.42(\mathrm{dd}, J=14.1,6.0 \mathrm{~Hz}, 1 \mathrm{H}), 1.28-1.25(\mathrm{~d}, J=16.2 \mathrm{~Hz}, 12 \mathrm{H}) ;{ }^{13} \mathrm{C}$ NMR (126 MHz, $\left.\mathrm{CDCl}_{3}\right) \delta 162.0(\mathrm{~d}, J=245.6 \mathrm{~Hz}), 141.9,139.3(\mathrm{~d}, J=3.1 \mathrm{~Hz}), 128.6$, $128.5,128.2(\mathrm{~d}, J=8.0 \mathrm{~Hz}), 126.0,115.2(\mathrm{~d}, J=21.4 \mathrm{~Hz}), 84.1,58.6,37.7,35.1,30.8,25.0$, 24.9; ${ }^{19} \mathrm{~F} \mathrm{NMR}\left(471 \mathrm{MHz}, \mathrm{CDCl}_{3}\right) \delta-115.4 ;{ }^{11} \mathrm{~B} \mathrm{NMR}\left(161 \mathrm{MHz}, \mathrm{CDCl}_{3}\right) \delta 33.4 ; \mathrm{HRMS}$ (EI) $\mathrm{m} / \mathrm{z}$ calcd. for $\mathrm{C}_{24} \mathrm{H}_{34} \mathrm{BFN}_{2} \mathrm{O}_{4} \mathrm{~S}$ [M] $]^{+}: 476.2316$, found: 476.2315; CHIRALCEL IB, $n$ hexane : $i-\mathrm{PrOH}=98: 2,1 \mathrm{~mL} / \mathrm{min}, 25^{\circ} \mathrm{C}, \lambda=220 \mathrm{~nm}$; $\mathrm{t}_{\mathrm{R}} 5.9 \mathrm{~min}$ (minor), $\mathrm{t}_{\mathrm{R}} 7.2 \mathrm{~min}$ (major); Analogous mixtures of enantiomers were prepared using $( \pm)-\mathbf{L 1}$ as a ligand under the standard reaction procedure.

[Racemic 3c]

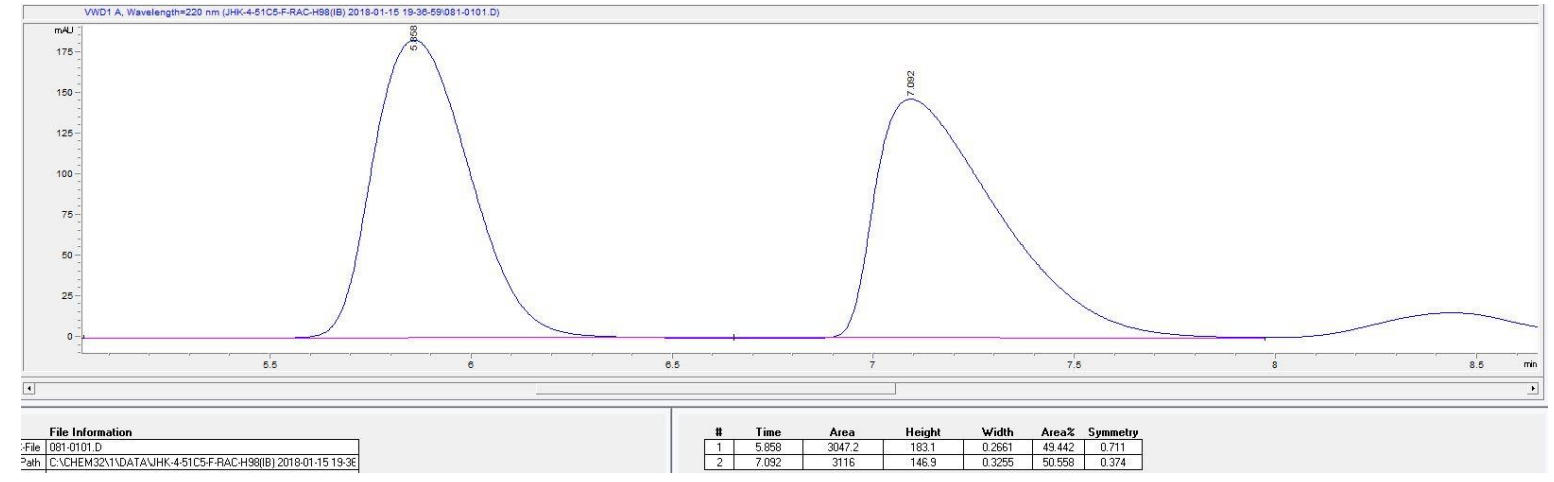

[Enantioenriched 3c]

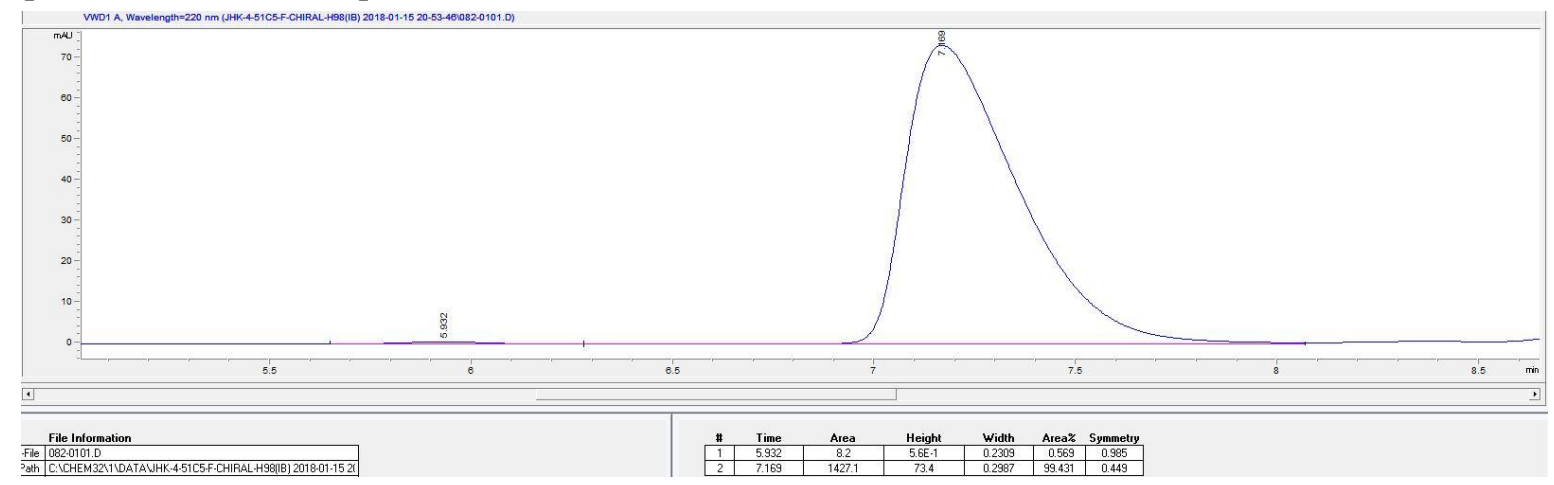


<smiles>CNS(=O)(=O)N[C@H](c1ccc(Cl)cc1)[C@@H](CCc1ccccc1)c1ccccc1</smiles>

$N, N$-Dimethyl- $N^{\prime}$-[(1S,2R)-1-(4-chlorophenyl)-4-phenyl-2-(4,4,5,5-tetramethyl-1,3,2dioxaborolan-2-yl)butyl]sulfamide (Table 2, 3d): $\mathrm{CuBr}(1.4 \mathrm{mg}, 0.010 \mathrm{mmol}, 5.0 \mathrm{~mol} \%)$, L1 (7.2 mg, $0.020 \mathrm{mmol}, 10 \mathrm{~mol} \%$ ), LiOtBu (32 mg, $0.40 \mathrm{mmol}$ ), 1,1-diboryl-3phenylpropane $(112 \mathrm{mg}, 0.30 \mathrm{mmol}), N, N$-dimethyl- $N^{\prime}$-[(4-chlorophenyl)methylene)] sulfamide (49 mg, $0.20 \mathrm{mmol})$ and 1,4-dioxane/toluene $(0.40 \mathrm{~mL}, \mathrm{v} / \mathrm{v} 1: 1)$ at $50{ }^{\circ} \mathrm{C}$ for $24 \mathrm{~h}$; Major diastereomer; ${ }^{1} \mathrm{H}-\mathrm{NMR}$ analysis of the crude mixture indicated 15:1 diastereoselectivity. The crude mixture was purified by silica gel column chromatography $\left(\mathrm{Et}_{2} \mathrm{O}: n\right.$-hexane $=1: 2$ to $\left.1: 1\right)$ to give the corresponding $\beta$-aminoboronate ester $\mathbf{3 d}$ as a white solid (75 mg, 76\%, 99\% ee); $[\alpha]_{\mathrm{D}}^{22}-12.6\left(c 0.73, \mathrm{CH}_{2} \mathrm{Cl}_{2}\right) ;{ }^{1} \mathrm{H} \mathrm{NMR}\left(500 \mathrm{MHz}, \mathrm{CDCl}_{3}\right)$ $\delta 7.28-7.26(\mathrm{dd}, J=7.1,5.6 \mathrm{~Hz}, 4 \mathrm{H}), 7.21-7.17(\mathrm{dd}, J=13.0,7.9 \mathrm{~Hz}, 5 \mathrm{H}), 5.52-5.51$ $(\mathrm{d}, J=8.1 \mathrm{~Hz}, 1 \mathrm{H}), 4.49-4.46(\mathrm{dd}, J=7.6,6.0 \mathrm{~Hz}, 1 \mathrm{H}), 2.76-2.65(\mathrm{~m}, 2 \mathrm{H}), 2.52(\mathrm{~s}, 6 \mathrm{H})$, $1.88-1.74(\mathrm{~m}, 2 \mathrm{H}), 1.44-1.38(\mathrm{~m}, 1 \mathrm{H}), 1.25-1.22(\mathrm{~d}, J=14.9 \mathrm{~Hz}, 12 \mathrm{H}) ;{ }^{13} \mathrm{C} \mathrm{NMR}(126$ $\left.\mathrm{MHz}, \mathrm{CDCl}_{3}\right) \delta 142.0,141.8,133.0,128.6,128.5,128.5,128.0,126.0,84.1,58.6,37.8,35.0$, 30.8, 25.0, 24.9; ${ }^{11} \mathrm{~B}$ NMR (161 MHz, $\mathrm{CDCl}_{3}$ ) $\delta$ 33.9; HRMS (EI) $\mathrm{m} / \mathrm{z}$ calcd. for $\mathrm{C}_{24} \mathrm{H}_{34} \mathrm{BClN}_{2} \mathrm{O}_{4} \mathrm{~S}[\mathrm{M}]^{+}: 492.2021$, found: 492.2019; CHIRALCEL IB, $n$-hexane $: i$-PrOH $=$ $98: 2,1 \mathrm{~mL} / \mathrm{min}, 25{ }^{\circ} \mathrm{C}, \lambda=220 \mathrm{~nm}$; $\mathrm{t}_{\mathrm{R}} 6.7 \mathrm{~min}$ (minor), $\mathrm{t}_{\mathrm{R}} 8.2 \mathrm{~min}$ (major); Analogous mixtures of enantiomers were prepared using $( \pm)-\mathbf{L} \mathbf{1}$ as a ligand under the standard reaction procedure.

[Racemic 3d]

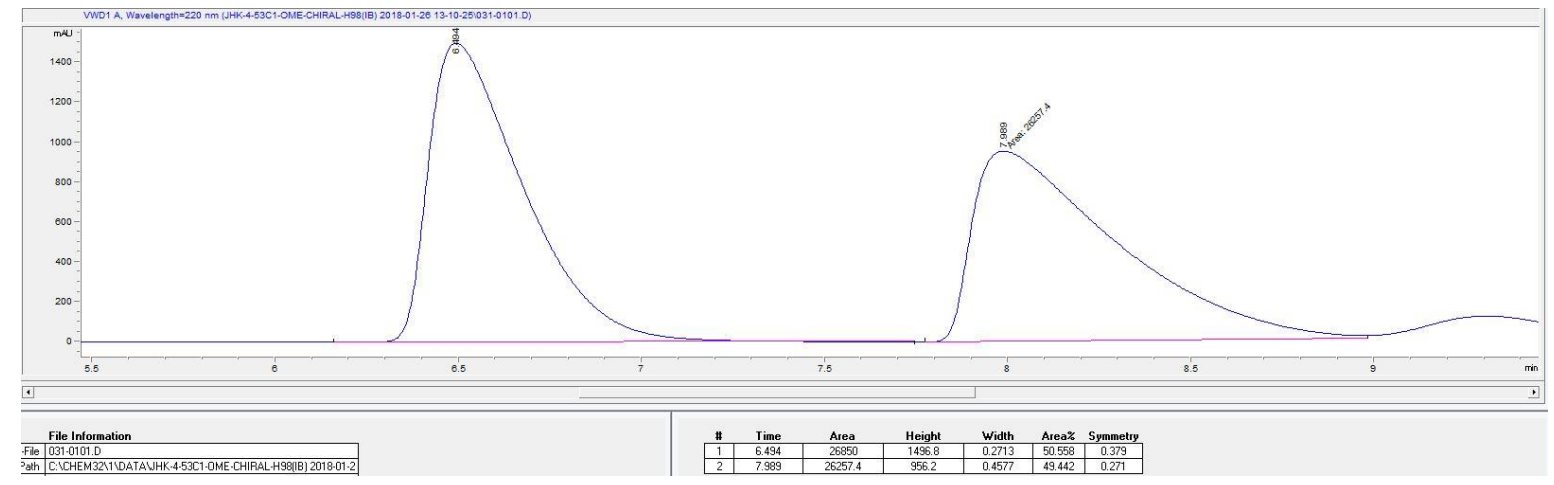

[Enantioenriched 3d]

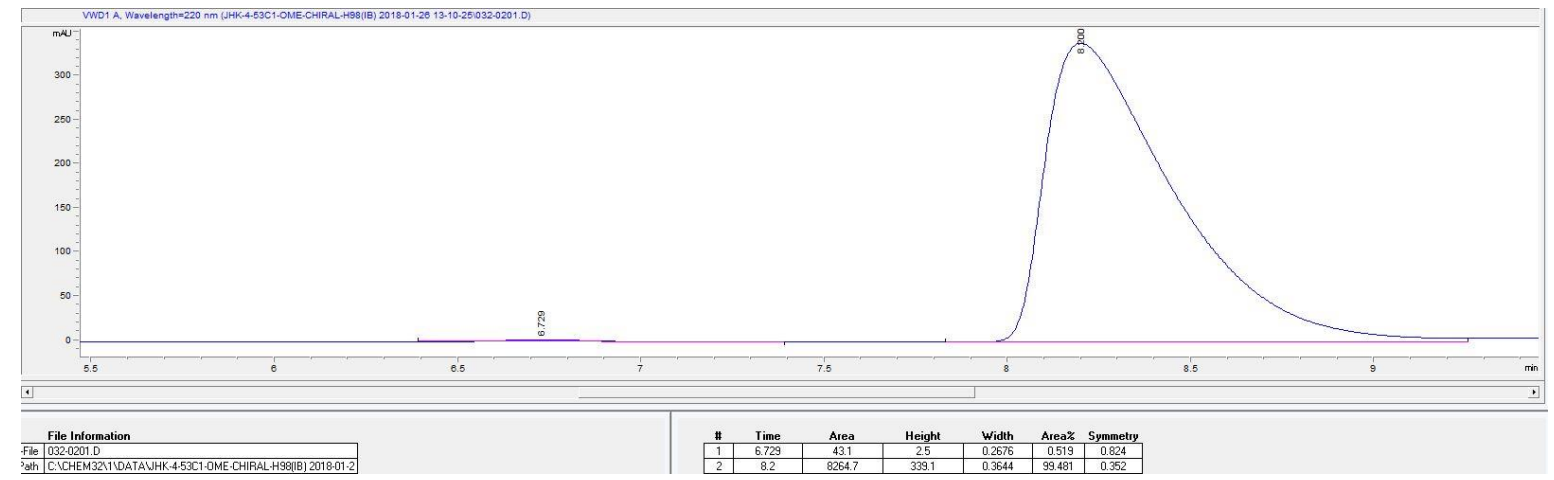


<smiles>CNS(=O)(=O)NC(c1ccc(Br)cc1)[C@H](CCc1ccccc1)c1ccccc1</smiles>

\section{$N, N$-Dimethyl- $N^{\prime}$-[(1S,2R)-1-(4-bromophenyl)-4-phenyl-2-(4,4,5,5-tetramethyl-1,3,2-}

dioxaborolan-2-yl)butyl]sulfamide (Table 2, 3e): $\mathrm{CuBr}(1.4 \mathrm{mg}, 0.010 \mathrm{mmol}, 5.0 \mathrm{~mol} \%$ ), L1 (7.2 mg, $0.020 \mathrm{mmol}, 10 \mathrm{~mol} \%$ ), LiOtBu (32 mg, $0.40 \mathrm{mmol}$ ), 1,1-diboryl-3phenylpropane $(112 \mathrm{mg}, 0.30 \mathrm{mmol}), N, N$-dimethyl- $N^{\prime}$-[(4-bromophenyl)methylene)] sulfamide $(58 \mathrm{mg}, 0.20 \mathrm{mmol})$ and 1,4-dioxane/toluene $(0.40 \mathrm{~mL}, \mathrm{v} / \mathrm{v} 1: 1)$ at $50{ }^{\circ} \mathrm{C}$ for $24 \mathrm{~h}$. ${ }^{1} \mathrm{H}-\mathrm{NMR}$ analysis of the crude mixture indicated 14:1 diastereoselectivity. The crude mixture was purified by silica gel column chromatography $\left(\mathrm{Et}_{2} \mathrm{O}: n\right.$-hexane $=1: 2$ to $\left.1: 1\right)$ to give the corresponding $\beta$-aminoboronate ester $3 \mathbf{e}$ as a white solid (87 $\mathrm{mg}, 81 \%, 98 \%$ ee); $[\alpha]_{\mathrm{D}}^{22}-15.7\left(c \mathrm{0} .86, \mathrm{CH}_{2} \mathrm{Cl}_{2}\right)$; Major diastereomer; ${ }^{1} \mathrm{H} \mathrm{NMR}\left(500 \mathrm{MHz}, \mathrm{CDCl}_{3}\right) \delta 7.44-7.42$ $(\mathrm{d}, J=8.4 \mathrm{~Hz}, 2 \mathrm{H}), 7.29-7.26(\mathrm{~m}, 2 \mathrm{H}), 7.19-7.14(\mathrm{~m}, 5 \mathrm{H}), 5.53-5.51(\mathrm{~d}, J=8.1 \mathrm{~Hz}$, $1 \mathrm{H}), 4.47-4.45(\mathrm{dd}, J=8.0,5.7 \mathrm{~Hz}, 1 \mathrm{H}), 2.77-2.65(\mathrm{~m}, 2 \mathrm{H}), 2.52(\mathrm{~s}, 6 \mathrm{H}), 1.88-1.74(\mathrm{~m}$, $2 \mathrm{H}), 1.42-1.38(\mathrm{~m}, 1 \mathrm{H}), 1.25-1.22(\mathrm{~d}, J=14.4 \mathrm{~Hz}, 12 \mathrm{H}) ;{ }^{13} \mathrm{C} \mathrm{NMR}\left(126 \mathrm{MHz}, \mathrm{CDCl}_{3}\right) \delta$ 142.6, 141.8, 131.5, 128.6, 128.5, 128.3, 126.0, 121.0, 84.1, 58.6, 37.7, 35.0, 30.8, 25.0, 24.9; ${ }^{11} \mathrm{~B}$ NMR (161 MHz, $\mathrm{CDCl}_{3}$ ) $\delta 33.4$; HRMS (EI) m/z calcd. for $\mathrm{C}_{24} \mathrm{H}_{34} \mathrm{BBrN}_{2} \mathrm{O}_{4} \mathrm{~S}$ $[\mathrm{M}]^{+}:$536.1516, found: 536.1512; CHIRALCEL IB, $n$-hexane $: i$-PrOH $=98: 2,1 \mathrm{~mL} / \mathrm{min}$, $25{ }^{\circ} \mathrm{C}, \lambda=220 \mathrm{~nm} ; \mathrm{t}_{\mathrm{R}} 6.6 \mathrm{~min}$ (minor), $\mathrm{t}_{\mathrm{R}} 8.1 \mathrm{~min}$ (major); Analogous mixtures of enantiomers were prepared using $( \pm)-\mathbf{L} \mathbf{1}$ as a ligand under the standard reaction procedure.

[Racemic 3e]

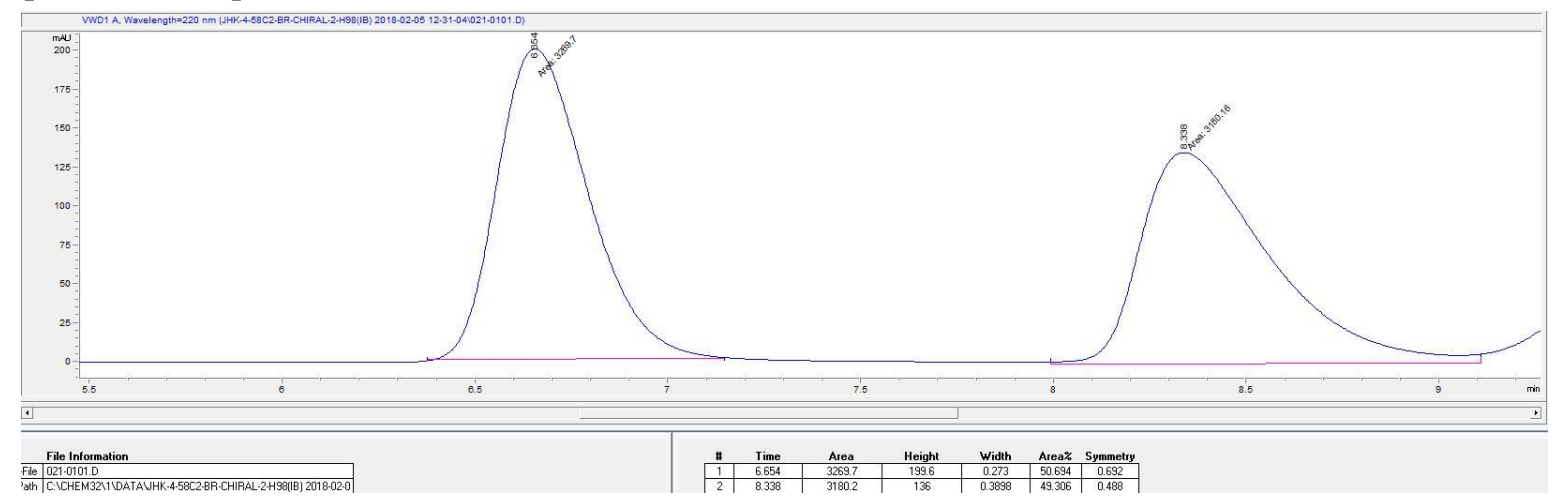

[Enantioenriched 3e]

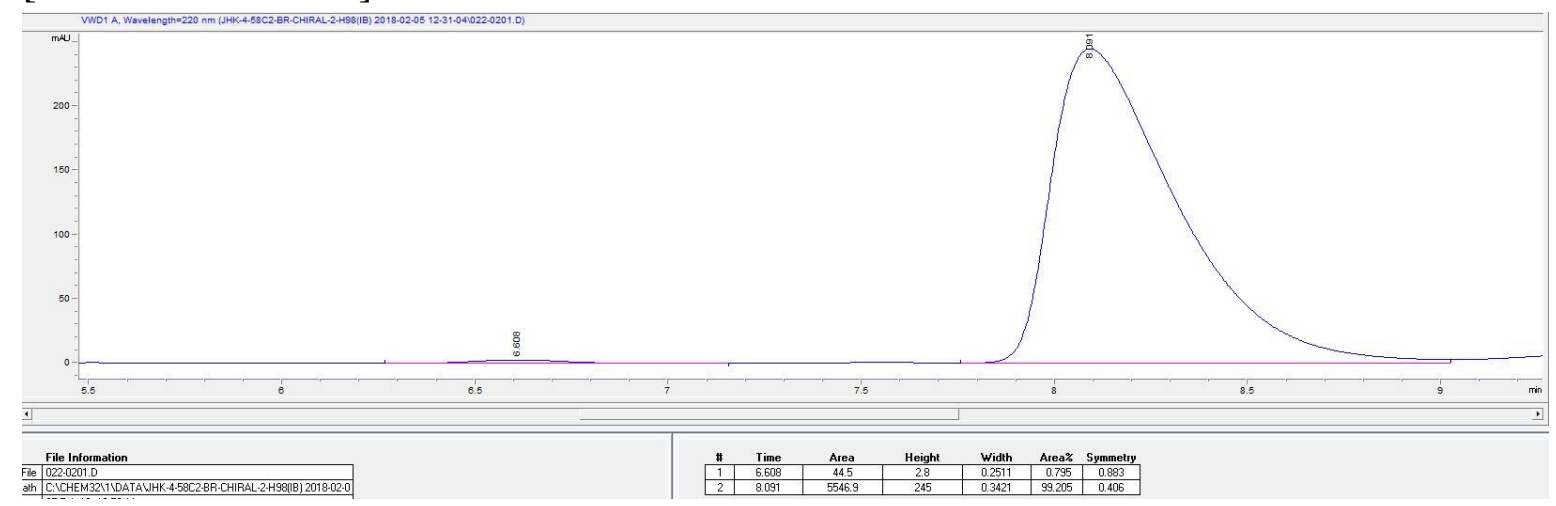


<smiles>Cc1ccc(C(NS(C)(=O)=O)[C@H](CCc2ccccc2)Cc2ccccc2)cc1</smiles>

$N, N$-Dimethyl- $N^{\prime}$-[(1S,2R)-4-phenyl-2-(4,4,5,5-tetramethyl-1,3,2-dioxaborolan-2-yl)-1(p-tolyl)butyl]sulfamide (Table 2, 3f): CuBr (1.4 mg, $0.010 \mathrm{mmol}, 5.0 \mathrm{~mol} \%)$, L1 (7.2 mg, $0.020 \mathrm{mmol}, 10 \mathrm{~mol} \%$ ), LiOt $\mathrm{Bu}$ (32 mg, $0.40 \mathrm{mmol}$ ), 1,1-diboryl-3-phenylpropane (149 mg, $0.40 \mathrm{mmol}), N, N$-dimethyl- $N$ '-[(4-methylphenyl)methylene)]sulfamide (45 mg, $0.20 \mathrm{mmol})$ and 1,4-dioxane/toluene $(0.40 \mathrm{~mL}, \mathrm{v} / \mathrm{v} 1: 1)$ at $50{ }^{\circ} \mathrm{C}$ for $24 \mathrm{~h} .{ }^{1} \mathrm{H}-\mathrm{NMR}$ analysis of the crude mixture indicated 18:1 diastereoselectivity. The crude mixture was purified by silica gel column chromatography $\left(\mathrm{Et}_{2} \mathrm{O}: n\right.$-hexane $=1: 2$ to $\left.1: 1\right)$ to give the corresponding $\beta$ aminoboronate ester $3 \mathbf{f}$ as a yellow solid (64 mg, 68\%, 98\% ee); $[\alpha]_{\mathrm{D}}^{22}-4.6\left(c 0.50, \mathrm{CH}_{2} \mathrm{Cl}_{2}\right)$; Major diastereomer; ${ }^{1} \mathrm{H}$ NMR $\left(500 \mathrm{MHz}, \mathrm{CDCl}_{3}\right) \delta 7.30-7.27(\mathrm{t}, J=7.5 \mathrm{~Hz}, 2 \mathrm{H}), 7.21-$ $7.20(\mathrm{~d}, J=7.4 \mathrm{~Hz}, 3 \mathrm{H}), 7.17-7.16(\mathrm{~d}, J=8.1 \mathrm{~Hz}, 2 \mathrm{H}), 7.13-7.11(\mathrm{~d}, J=8.0 \mathrm{~Hz}, 2 \mathrm{H})$, $5.52-5.51(\mathrm{~d}, J=8.3 \mathrm{~Hz}, 1 \mathrm{H}), 4.50-4.47(\mathrm{dd}, J=8.2,6.0 \mathrm{~Hz}, 1 \mathrm{H}), 2.78-2.65(\mathrm{~m}, 2 \mathrm{H})$, $2.51(\mathrm{~s}, 6 \mathrm{H}), 2.34(\mathrm{~s}, 3 \mathrm{H}), 1.89-1.77(\mathrm{~m}, 2 \mathrm{H}), 1.47-1.43(\mathrm{dd}, J=14.2,6.6 \mathrm{~Hz}, 1 \mathrm{H}), 1.29$ $-1.25(\mathrm{~d}, J=18.5 \mathrm{~Hz}, 12 \mathrm{H}) ;{ }^{13} \mathrm{C} \mathrm{NMR}\left(126 \mathrm{MHz}, \mathrm{CDCl}_{3}\right) \delta 142.1,140.5,136.8,129.1$, 128.6, 128.4, 126.5, 125.9, 84.0, 59.0, 37.7, 35.2, 30.9, 25.0, 25.0, 21.2; ${ }^{11} \mathrm{~B}$ NMR $(161 \mathrm{MHz}$, $\mathrm{CDCl}_{3}$ ) $\delta 33.7$; HRMS (EI) m/z calcd. for $\mathrm{C}_{25} \mathrm{H}_{37} \mathrm{BN}_{2} \mathrm{O}_{4} \mathrm{~S}[\mathrm{M}]^{+}$: 472.2567, found: 473.2569; CHIRALCEL IB, $n$-hexane $: i$-PrOH $=98: 2,1 \mathrm{~mL} / \mathrm{min}, 25{ }^{\circ} \mathrm{C}, \lambda=220 \mathrm{~nm} ; \mathrm{t}_{\mathrm{R}} 5.3 \mathrm{~min}$ (minor), $\mathrm{t}_{\mathrm{R}} 6.4 \mathrm{~min}$ (major); Analogous mixtures of enantiomers were prepared using ( \pm )-L1 as a ligand under the standard reaction procedure.

[Racemic 3f]

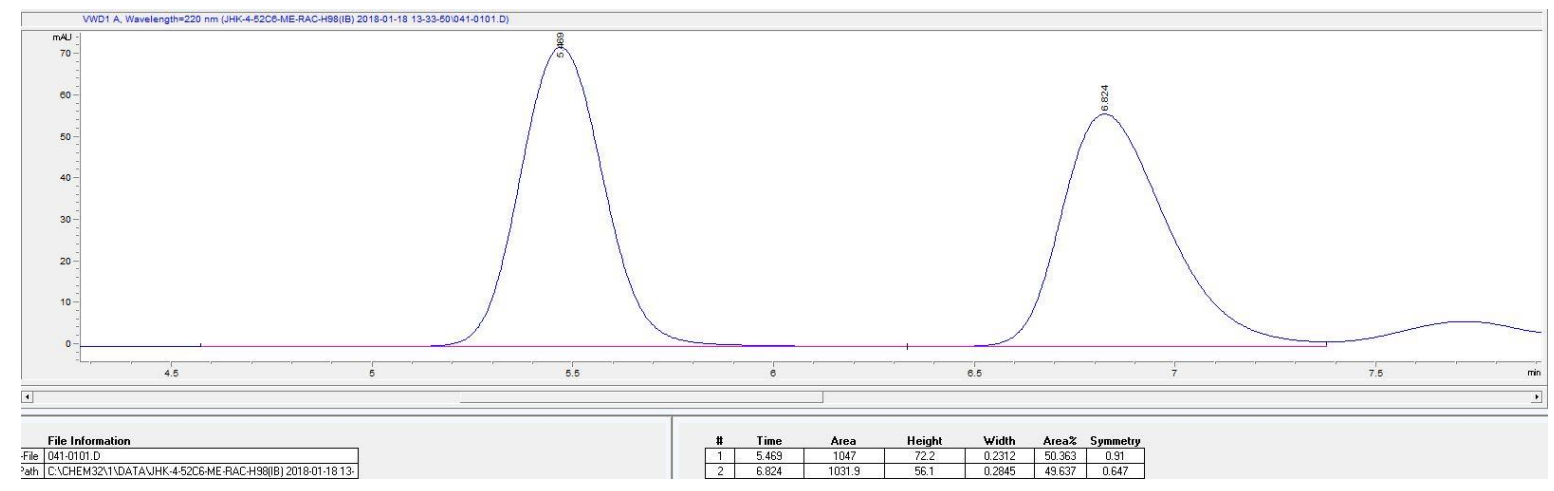

[Enantioenriched 3f]

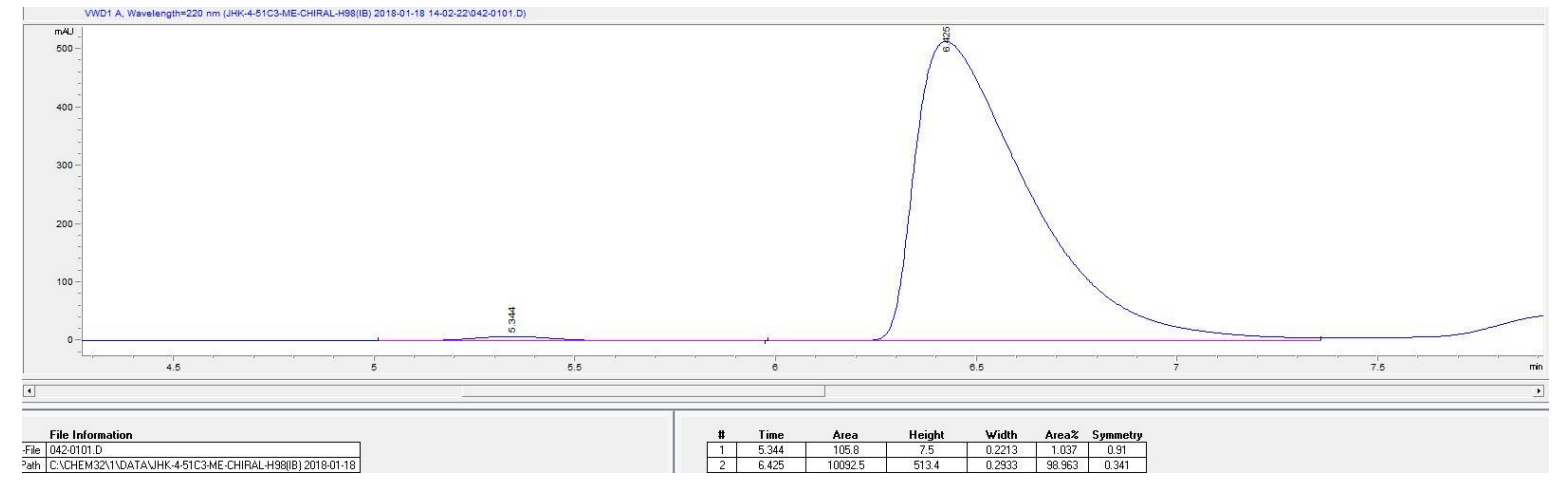


<smiles>COc1ccc([C@H](NS(=O)(=O)c2ccccc2)[C@H](CCc2ccccc2)c2ccccc2)cc1</smiles>

\section{$N, N$-Dimethyl- $N^{\prime}$-[(1S,2R)-1-(4-methoxyphenyl)-4-phenyl-2-(4,4,5,5-tetramethyl-}

1,3,2-dioxaborolan-2-yl)butyl]sulfamide (Table 2, 3g): $\mathrm{CuBr}$ (1.4 mg, $0.010 \mathrm{mmol}, 5.0$ mol \%), L1 (7.2 mg, $0.020 \mathrm{mmol}, 10 \mathrm{~mol} \%)$, LiOtBu (32 mg, $0.40 \mathrm{mmol}), 1$,1-diboryl-3phenylpropane (149 mg, $0.40 \mathrm{mmol}), N, N$-dimethyl- $N$ '-[(4-methoxyphenyl)methylene)] sulfamide (49 mg, $0.20 \mathrm{mmol})$ and 1,4-dioxane/toluene $(0.40 \mathrm{~mL}, \mathrm{v} / \mathrm{v} 1: 1)$ at $50{ }^{\circ} \mathrm{C}$ for $24 \mathrm{~h}$. ${ }^{1} \mathrm{H}-\mathrm{NMR}$ analysis of the crude mixture indicated 14:1 diastereoselectivity. The crude mixture was purified by silica gel column chromatography $\left(\mathrm{Et}_{2} \mathrm{O}: n\right.$-hexane $=1: 2$ to $1: 1$ ) to give the corresponding $\beta$-aminoboronate ester $3 \mathrm{~g}$ as a white solid (62 $\mathrm{mg}, 63 \%, 96 \%$ ee); $[\alpha]_{\mathrm{D}}^{22}-4.7\left(c 0.53, \mathrm{CH}_{2} \mathrm{Cl}_{2}\right)$; Major diastereomer; ${ }^{1} \mathrm{H} \mathrm{NMR}\left(500 \mathrm{MHz}, \mathrm{CDCl}_{3}\right) \delta 7.27-7.24$ (t, $J=7.5 \mathrm{~Hz}, 2 \mathrm{H}), 7.18-7.16(\mathrm{dd}, J=7.9,3.5 \mathrm{~Hz}, 5 \mathrm{H}), 6.84-6.82(\mathrm{~d}, J=8.6 \mathrm{~Hz}, 2 \mathrm{H})$, $5.45-5.43(\mathrm{~d}, J=8.1 \mathrm{~Hz}, 1 \mathrm{H}), 4.46-4.43(\mathrm{~m}, 1 \mathrm{H}), 3.79(\mathrm{~s}, 1 \mathrm{H}), 2.73-2.64(\mathrm{~m}, 2 \mathrm{H}), 2.48$ (s, 6H), $1.85-1.71(\mathrm{~m}, 2 \mathrm{H}), 1.44-1.39(\mathrm{dd}, J=14.6,6.6 \mathrm{~Hz}, 1 \mathrm{H}), 1.26-1.23(\mathrm{~d}, J=16.5$ $\mathrm{Hz}, 12 \mathrm{H}) ;{ }^{13} \mathrm{C} \mathrm{NMR}\left(126 \mathrm{MHz}, \mathrm{CDCl}_{3}\right) \delta 158.7,142.0,135.5,128.5,128.3,127.6,125.8$, 113.7, 83.9, 58.6, 55.3, 37.6, 35.1, 30.8, 24.9, 24.9; ${ }^{11} \mathrm{~B}$ NMR (161 $\left.\mathrm{MHz}, \mathrm{CDCl}_{3}\right) \delta 34.0$; HRMS (EI) $\mathrm{m} / \mathrm{z}$ calcd. for $\mathrm{C}_{25} \mathrm{H}_{37} \mathrm{BN}_{2} \mathrm{O}_{5} \mathrm{~S}[\mathrm{M}]^{+}:$488.2516, found: 488.2514; CHIRALCEL $\mathrm{IB}, n$-hexane $: i-\mathrm{PrOH}=98: 2,1 \mathrm{~mL} / \mathrm{min}, 25^{\circ} \mathrm{C}, \lambda=220 \mathrm{~nm} ; \mathrm{t}_{\mathrm{R}} 8.8 \mathrm{~min}$ (minor), $\mathrm{t}_{\mathrm{R}} 11.1 \mathrm{~min}$ (major); Analogous mixtures of enantiomers were prepared using $( \pm)-\mathbf{L} \mathbf{1}$ as a ligand under the standard reaction procedure.

[Racemic 3g]

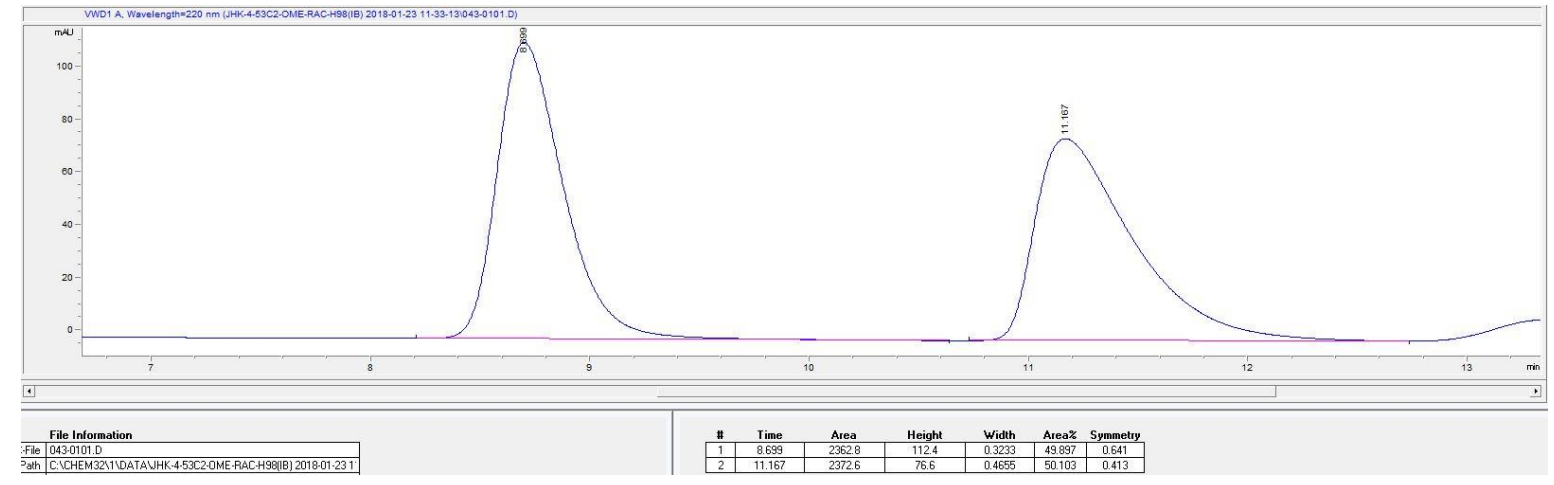

[Enantioenriched 3g]

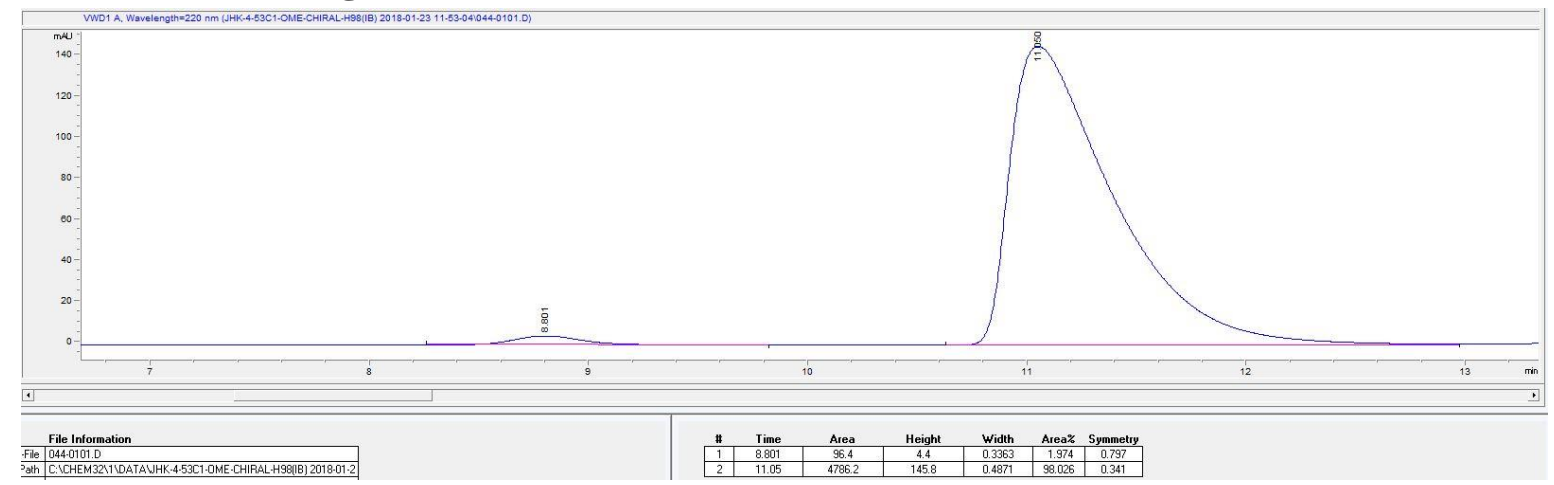


<smiles>CNS(=O)(=O)NC(c1cccc(Br)c1)C(CCc1ccccc1)c1ccccc1</smiles>

$N, N$-Dimethyl- $N^{\prime}-[(1 S, 2 R)-1-(3-b r o m o p h e n y l)-4-p h e n y l-2-(4,4,5,5-t e t r a m e t h y l-1,3,2-$ dioxaborolan-2-yl)butyl]sulfamide (Table 2, 3h): $\mathrm{CuBr}(1.4 \mathrm{mg}, 0.010 \mathrm{mmol}, 5.0 \mathrm{~mol} \%$ ), L1 (7.2 mg, $0.020 \mathrm{mmol}, 10 \mathrm{~mol} \%$ ), LiOtBu (32 mg, $0.40 \mathrm{mmol}$ ), 1,1-diboryl-3phenylpropane $(112 \mathrm{mg}, 0.30 \mathrm{mmol}), N, N$-dimethyl- $N$ '-[(3-bromophenyl)methylene)] sulfamide $(58 \mathrm{mg}, 0.20 \mathrm{mmol})$ and 1,4-dioxane/toluene $(0.40 \mathrm{~mL}, \mathrm{v} / \mathrm{v} 1: 1)$ at $50{ }^{\circ} \mathrm{C}$ for $24 \mathrm{~h}$. ${ }^{1} \mathrm{H}-\mathrm{NMR}$ analysis of the crude mixture indicated 14:1 diastereoselectivity. The crude mixture was purified by silica gel column chromatography $\left(\mathrm{Et}_{2} \mathrm{O}: n\right.$-hexane $=1: 2$ to $\left.1: 1\right)$ to give the corresponding $\beta$-aminoboronate ester $\mathbf{3 h}$ as a yellow solid $(67 \mathrm{mg}, 62 \%, 99 \%$ ee); $[\alpha]_{\mathrm{D}}^{23}-9.3\left(c \mathrm{0.58}, \mathrm{CH}_{2} \mathrm{Cl}_{2}\right)$; Major diastereomer; ${ }^{1} \mathrm{H} \mathrm{NMR}\left(500 \mathrm{MHz}, \mathrm{CDCl}_{3}\right) \delta 7.47(\mathrm{~s}$, $1 \mathrm{H}), 7.37-7.35(\mathrm{dt}, J=7.0,1.9 \mathrm{~Hz}, 1 \mathrm{H}), 7.30-7.27(\mathrm{~m}, 2 \mathrm{H}), 7.20-7.16(\mathrm{dd}, J=15.0,7.3$ $\mathrm{Hz}, 5 \mathrm{H}), 5.58-5.56(\mathrm{~d}, J=8.2 \mathrm{~Hz}, 1 \mathrm{H}), 4.50-4.47$ (dd, $J=8.2,5.2 \mathrm{~Hz}, 1 \mathrm{H}), 2.80-2.66$ $(\mathrm{m}, 2 \mathrm{H}), 2.54(\mathrm{~s}, 6 \mathrm{H}), 1.91-1.80(\mathrm{~m}, 2 \mathrm{H}), 1.43-1.39(\mathrm{~m}, 1 \mathrm{H}), 1.26-1.22(\mathrm{~d}, J=21.1 \mathrm{~Hz}$, $12 \mathrm{H}) ;{ }^{13} \mathrm{C} \mathrm{NMR}\left(126 \mathrm{MHz}, \mathrm{CDCl}_{3}\right) \delta 146.0,141.8,130.4,130.1,129.5,128.6,128.5,126.0$, 125.4, 122.5, 84.2, 58.6, 37.8, 35.0, 30.8, 25.0, 24.9; ${ }^{11} \mathrm{~B}$ NMR (161 MHz, $\left.\mathrm{CDCl}_{3}\right) \delta 33.9$; HRMS (EI) $\mathrm{m} / \mathrm{z}$ calcd. for $\mathrm{C}_{24} \mathrm{H}_{34} \mathrm{BBrN}_{2} \mathrm{O}_{4} \mathrm{~S} \quad[\mathrm{M}]^{+}$: 536.1516, found: 536.1519; CHIRALCEL IB, $n$-hexane $: i$-PrOH $=98: 2,1 \mathrm{~mL} / \mathrm{min}, 25{ }^{\circ} \mathrm{C}, \lambda=220 \mathrm{~nm} ; t_{\mathrm{R}} 5.2 \mathrm{~min}$ (minor), $\mathrm{t}_{\mathrm{R}} 6.1 \mathrm{~min}$ (major); Analogous mixtures of enantiomers were prepared using $( \pm$ )-L1 as a ligand under the standard reaction procedure.

\section{[Racemic 3h]}

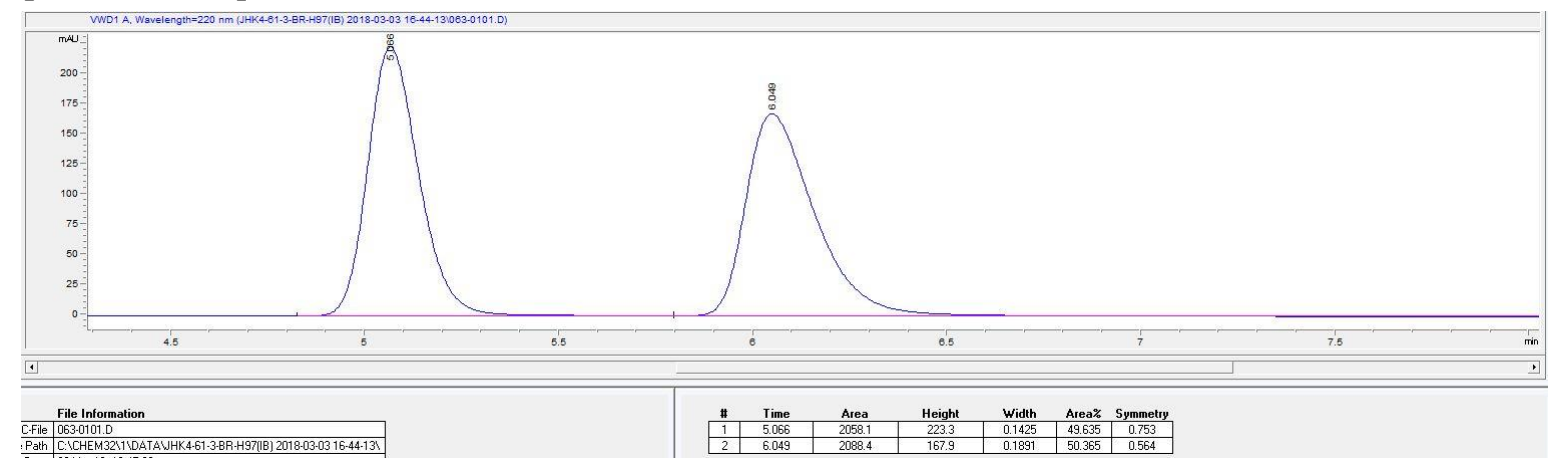

[Enantioenriched 3h]

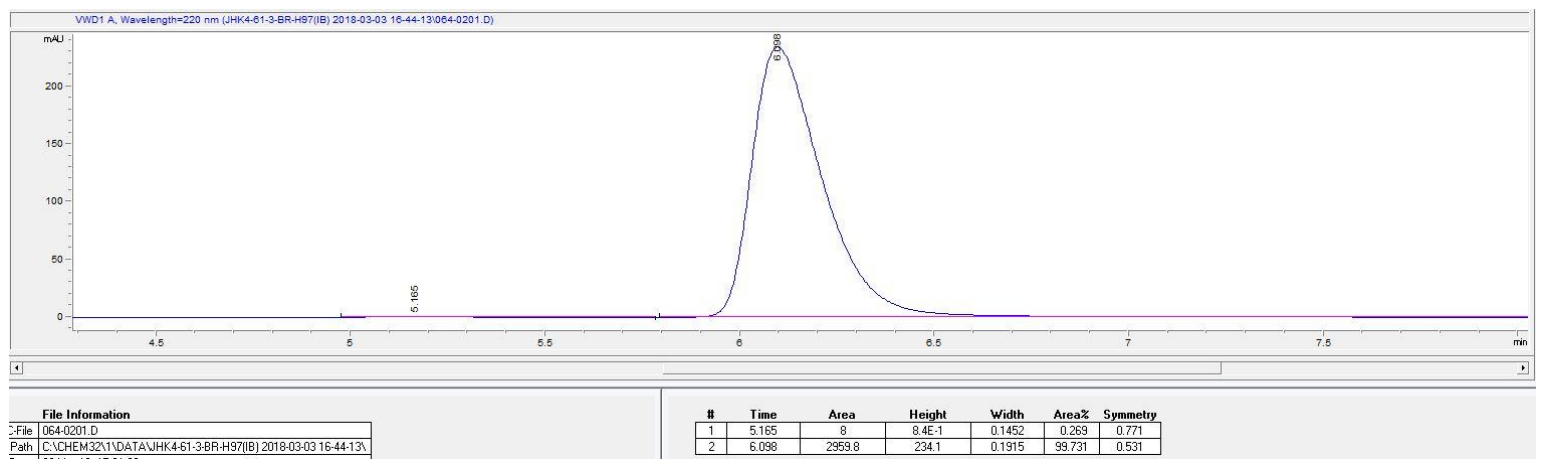


<smiles>Cc1cccc(C(NS(C)(=O)=O)C(CCc2ccccc2)c2ccccc2)c1</smiles>

$N, N$-Dimethyl- $N^{\prime}$-[(1S,2R)-4-phenyl-2-(4,4,5,5-tetramethyl-1,3,2-dioxaborolan-2-yl)-1(m-tolyl)butyl]sulfamide (Table 2, 3i): CuBr (1.4 mg, $0.010 \mathrm{mmol}, 5.0 \mathrm{~mol} \%$ ), L1 (7.2 $\mathrm{mg}, 0.020 \mathrm{mmol}, 10 \mathrm{~mol} \%), \mathrm{LiO} t \mathrm{Bu}$ (32 mg, $0.40 \mathrm{mmol}), 1$, 1-diboryl-3-phenylpropane (112 $\mathrm{mg}, 0.30 \mathrm{mmol}), N, N$-dimethyl- $N$ '-[(3-methylphenyl)methylene)]sulfamide (45 mg, 0.20 $\mathrm{mmol})$ and 1,4-dioxane/toluene $(0.40 \mathrm{~mL}$, v/v $1: 1)$ at $50{ }^{\circ} \mathrm{C}$ for $24 \mathrm{~h} .{ }^{1} \mathrm{H}-\mathrm{NMR}$ analysis of the crude mixture indicated $14: 1$ diastereoselectivity. The crude mixture was purified by silica gel column chromatography $\left(\mathrm{Et}_{2} \mathrm{O}: n\right.$-hexane $=1: 2$ to $\left.1: 1\right)$ to give the corresponding $\beta$-aminoboronate ester $3 \mathbf{i}$ as a yellow solid (70 mg, $74 \%, 97 \%$ ee); $[\alpha]_{\mathrm{D}}^{23}-4.1(c 0.63$, $\mathrm{CH}_{2} \mathrm{Cl}_{2}$ ); Major diastereomer; ${ }^{1} \mathrm{H} \mathrm{NMR}\left(500 \mathrm{MHz}, \mathrm{CDCl}_{3}\right) \delta 7.29-7.26(\mathrm{t}, J=7.5 \mathrm{~Hz}, 2 \mathrm{H})$, $7.20-7.16(\mathrm{~m}, 4 \mathrm{H}), 7.06-7.02(\mathrm{dd}, J=12.1,8.1 \mathrm{~Hz}, 3 \mathrm{H}), 5.55-5.53(\mathrm{~d}, J=8.4 \mathrm{~Hz}, 1 \mathrm{H})$, $4.48-4.46(\mathrm{dd}, J=8.4,5.6 \mathrm{~Hz}, 1 \mathrm{H}), 2.78-2.63(\mathrm{~m}, 2 \mathrm{H}), 2.49(\mathrm{~s}, 6 \mathrm{H}), 2.32(\mathrm{~s}, 3 \mathrm{H}), 1.89-$ $1.78(\mathrm{~m}, 2 \mathrm{H}), 1.46-1.42(\mathrm{~m}, 1 \mathrm{H}), 1.26-1.22(\mathrm{~d}, J=20.8 \mathrm{~Hz}, 12 \mathrm{H}) ;{ }^{13} \mathrm{C}$ NMR $(126 \mathrm{MHz}$, $\left.\mathrm{CDCl}_{3}\right) \delta 143.4,142.1,137.9,128.6,128.4,128.3,128.0,127.2,125.9,123.6,84.0,59.0$, 37.7, 35.1, 30.9, 25.0, 24.9, 21.6; ${ }^{11} \mathrm{~B}$ NMR (161 MHz, $\left.\mathrm{CDCl}_{3}\right) \delta 34.1 ; \mathrm{HRMS}(\mathrm{EI}) \mathrm{m} / \mathrm{z}$ calcd. for $\mathrm{C}_{25} \mathrm{H}_{37} \mathrm{BN}_{2} \mathrm{O}_{4} \mathrm{~S}[\mathrm{M}]^{+}:$472.2567, found: 472.2565; CHIRALCEL IB, $n$-hexane : $i$ $\mathrm{PrOH}=98: 2,1 \mathrm{~mL} / \mathrm{min}, 25{ }^{\circ} \mathrm{C}, \lambda=220 \mathrm{~nm}$; $\mathrm{t}_{\mathrm{R}} 5.0 \mathrm{~min}$ (minor), $\mathrm{t}_{\mathrm{R}} 5.9 \mathrm{~min}$ (major); Analogous mixtures of enantiomers were prepared using $( \pm)-\mathbf{L 1}$ as a ligand under the standard reaction procedure.

[Racemic 3i]

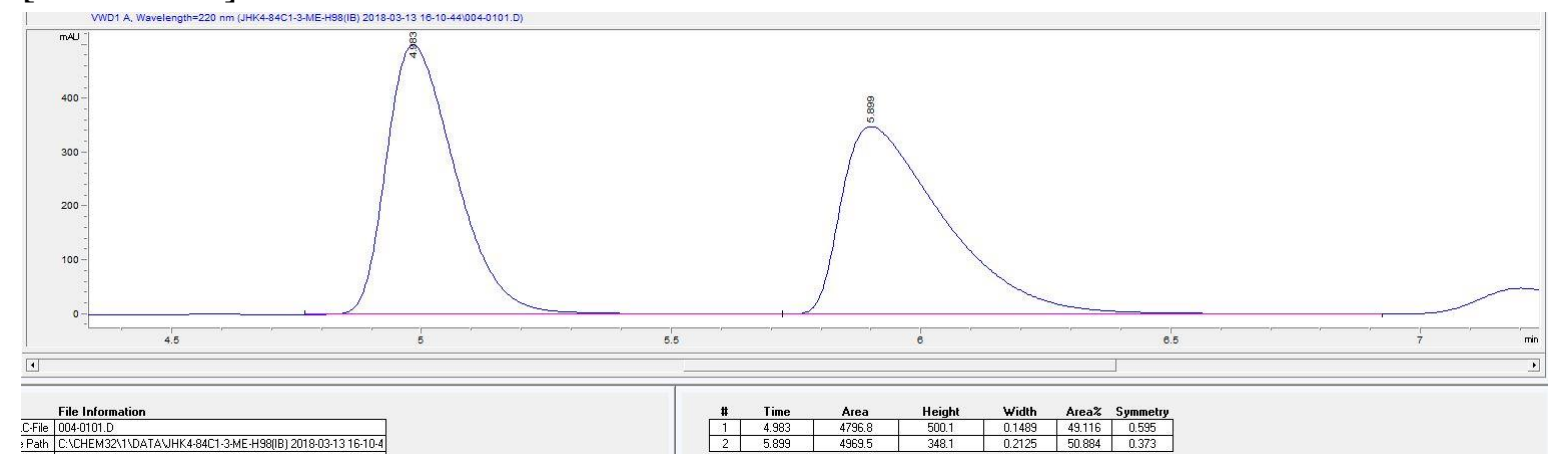

[Enantioenriched 3i]

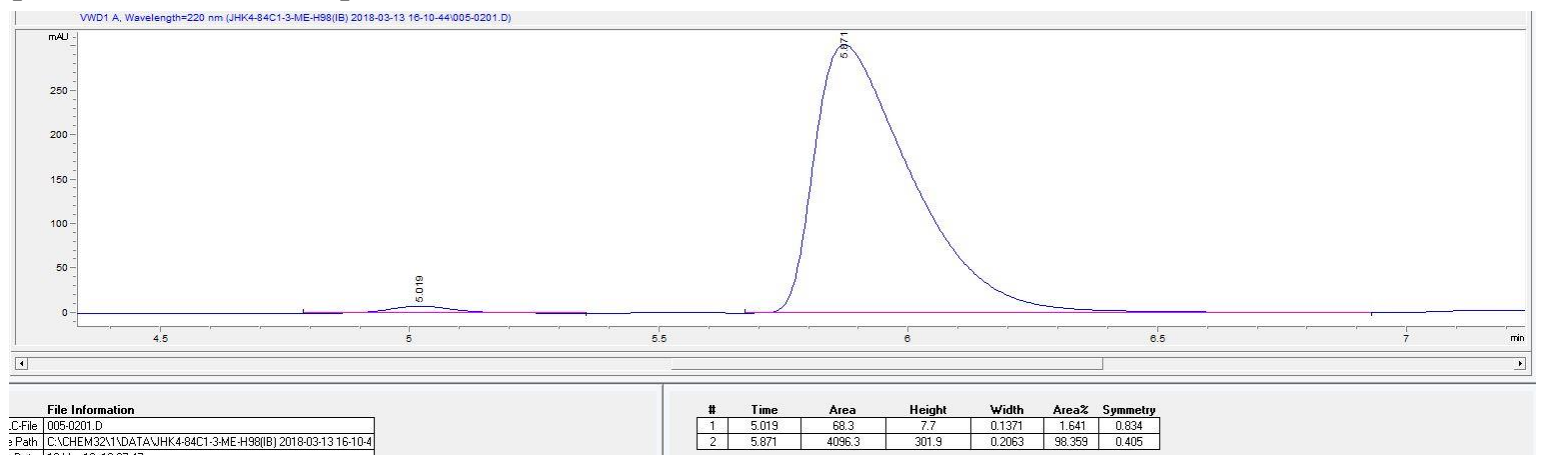


<smiles>COc1ccccc1C(NS(=O)(=O)OCc1ccccc1)C(CCc1ccccc1)Cc1ccccc1</smiles>

$N, N$-Dimethyl- $N^{\prime}$-[(1S,2R)-4-phenyl-2-(4,4,5,5-tetramethyl-1,3,2-dioxaborolan-2-yl)-1(o-tolyl)butyl]sulfamide (Table 2, 3j): $\mathrm{CuBr}(1.4 \mathrm{mg}, 0.010 \mathrm{mmol}, 5.0 \mathrm{~mol} \%)$, L1 (7.2 mg, $0.020 \mathrm{mmol}, 10 \mathrm{~mol} \%), \mathrm{LiO} t \mathrm{Bu}$ (32 mg, $0.40 \mathrm{mmol}), 1$,1-diboryl-3-phenylpropane (112 mg, $0.30 \mathrm{mmol}), N, N$-dimethyl- $N^{\prime}$-[(2-methylphenyl)methylene)]sulfamide (45 $\left.\mathrm{mg}, 0.20 \mathrm{mmol}\right)$ and 1,4-dioxane/toluene $(0.40 \mathrm{~mL}, \mathrm{v} / \mathrm{v} 1: 1)$ at $50{ }^{\circ} \mathrm{C}$ for $24 \mathrm{~h} .{ }^{1} \mathrm{H}-\mathrm{NMR}$ analysis of the crude mixture indicated 13:1 diastereoselectivity. The crude mixture was purified by silica gel column chromatography $\left(\mathrm{Et}_{2} \mathrm{O}: n\right.$-hexane $=1: 2$ to $\left.1: 1\right)$ to give the corresponding $\beta$ aminoboronate ester $\mathbf{3 j}$ as a white solid (56 mg, 59\%, 96\% ee); $[\alpha]_{\mathrm{D}}^{23}-2.0\left(c 0.51, \mathrm{CH}_{2} \mathrm{Cl}_{2}\right)$; Major diastereomer; ${ }^{1} \mathrm{H}$ NMR $\left(500 \mathrm{MHz}, \mathrm{CDCl}_{3}\right) \delta 7.40-7.38(\mathrm{~d}, J=7.7 \mathrm{~Hz}, 1 \mathrm{H}), 7.28-$ $7.25(\mathrm{t}, J=7.4 \mathrm{~Hz}, 2 \mathrm{H}), 7.22-7.16(\mathrm{~m}, 4 \mathrm{H}), 7.13-7.08(\mathrm{~m}, 2 \mathrm{H}), 5.69-5.67(\mathrm{~d}, J=8.3 \mathrm{~Hz}$, $1 \mathrm{H}), 4.77-4.74(\mathrm{dd}, J=8.2,4.9 \mathrm{~Hz}, 1 \mathrm{H}), 2.86-2.80(\mathrm{~m}, 1 \mathrm{H}), 2.70-2.65(\mathrm{~m}, 1 \mathrm{H}), 2.45(\mathrm{~s}$, $6 \mathrm{H}), 2.26(\mathrm{~s}, 3 \mathrm{H}), 1.95-1.83(\mathrm{~m}, 2 \mathrm{H}), 1.41-1.37(\mathrm{dd}, J=12.4,7.4 \mathrm{~Hz}, 1 \mathrm{H}), 1.28-1.23$ $(\mathrm{d}, J=25.6 \mathrm{~Hz}, 12 \mathrm{H}) ;{ }^{13} \mathrm{C} \mathrm{NMR}\left(126 \mathrm{MHz}, \mathrm{CDCl}_{3}\right) \delta 142.0,141.9,134.5,130.7,128.6$, $128.4,127.1,126.0,125.9,125.7,84.0,55.1,37.6,35.1,31.1,25.0,24.9,19.0 ;{ }^{11} \mathrm{~B} \mathrm{NMR}$ $\left(161 \mathrm{MHz}, \mathrm{CDCl}_{3}\right) \delta 33.5$; HRMS (EI) m/z calcd. for $\mathrm{C}_{25} \mathrm{H}_{37} \mathrm{BN}_{2} \mathrm{O}_{4} \mathrm{~S}[\mathrm{M}]^{+}: 472.2567$, found: 472.2570; CHIRALCEL IB, $n$-hexane : $i$-PrOH $=98: 2,1 \mathrm{~mL} / \mathrm{min}, 25^{\circ} \mathrm{C}, \lambda=220 \mathrm{~nm} ; \mathrm{t}_{\mathrm{R}} 4.5$ $\min$ (minor), $t_{R} 5.4$ min (major); Analogous mixtures of enantiomers were prepared using $( \pm)-\mathbf{L} \mathbf{1}$ as a ligand under the standard reaction procedure.

[Racemic 3j]

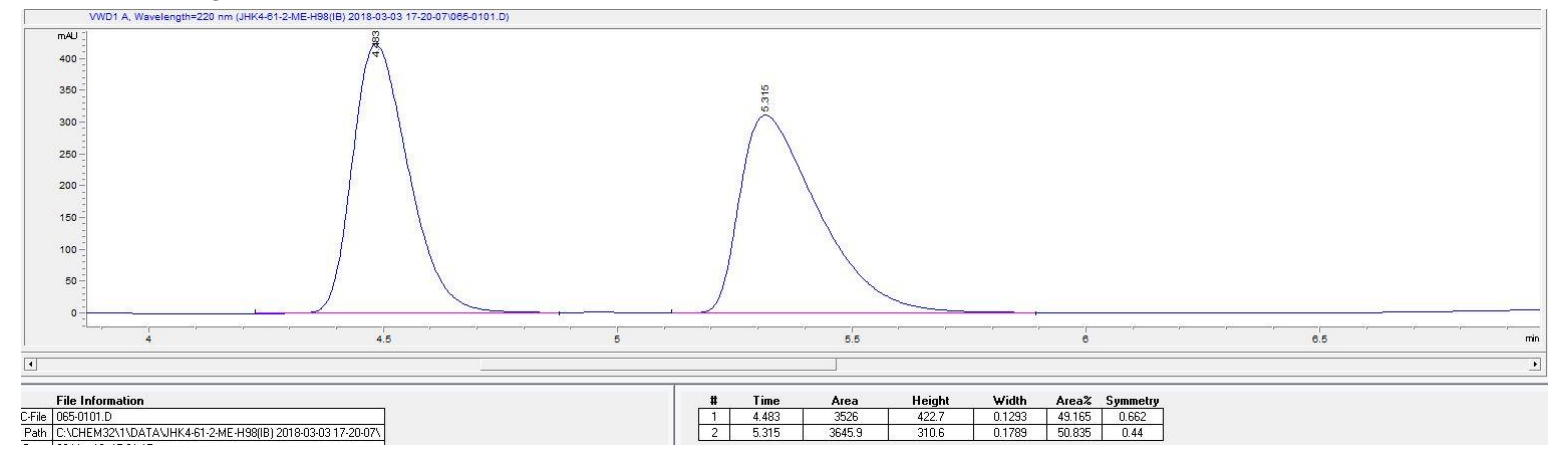

[Enantioenriched 3j]

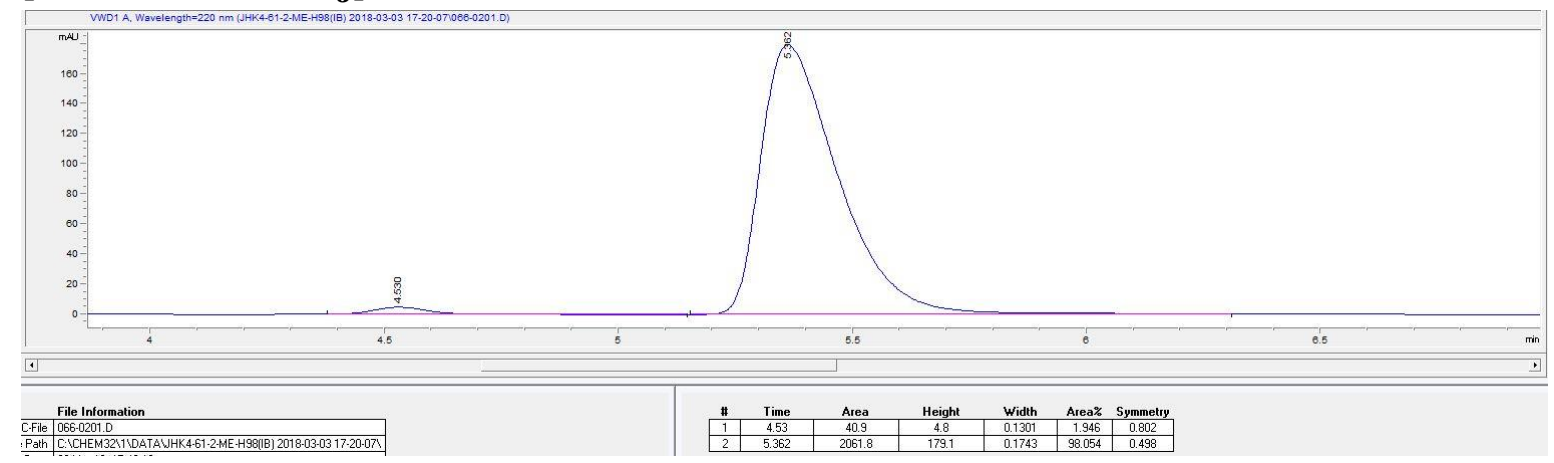


<smiles>CNS(=O)(=O)NC(c1ccc2ccccc2c1)C(CCc1ccccc1)c1ccccc1</smiles>

$N, N$-Dimethyl- $N^{\prime}$-[(1S,2R)-1-(naphthalen-2-yl)-4-phenyl-2-(4,4,5,5-tetramethyl-1,3,2dioxaborolan-2-yl)butyl]sulfamide (Table 2, 3k): $\mathrm{CuBr}$ (1.4 mg, $0.010 \mathrm{mmol}, 5.0 \mathrm{~mol} \%$ ), L1 (7.2 mg, $0.020 \mathrm{mmol}, 10 \mathrm{~mol} \%$ ), LiOtBu (32 mg, $0.40 \mathrm{mmol}$ ), 1,1-diboryl-3phenylpropane $(112 \mathrm{mg}, \quad 0.30 \mathrm{mmol}), N, N$-dimethyl- $N^{\prime}-[(2$-naphthalenyl)methylene)] sulfamide $(53 \mathrm{mg}, 0.20 \mathrm{mmol})$ and 1,4-dioxane/toluene $(0.40 \mathrm{~mL}, \mathrm{v} / \mathrm{v} 1: 1)$ at $50{ }^{\circ} \mathrm{C}$ for $48 \mathrm{~h}$. ${ }^{1} \mathrm{H}-\mathrm{NMR}$ analysis of the crude mixture indicated 11:1 diastereoselectivity. The crude mixture was purified by silica gel column chromatography $\left(\mathrm{Et}_{2} \mathrm{O}: n\right.$-hexane $=1: 2$ to $\left.1: 1\right)$ to give the corresponding $\beta$-aminoboronate ester 3k as a white solid (77 $\mathrm{mg}, 76 \%, 99 \%$ ee); $[\alpha]_{\mathrm{D}}^{23}-15.8\left(c \mathrm{0} .73, \mathrm{CH}_{2} \mathrm{Cl}_{2}\right)$; Major diastereomer; ${ }^{1} \mathrm{H} \mathrm{NMR}\left(500 \mathrm{MHz}, \mathrm{CDCl}_{3}\right) \delta 7.84-7.78$ $(\mathrm{m}, 3 \mathrm{H}), 7.74(\mathrm{~s}, 1 \mathrm{H}), 7.51-7.46(\mathrm{~m}, 2 \mathrm{H}), 7.41-7.39(\mathrm{dd}, J=8.4,1.2 \mathrm{~Hz}, 1 \mathrm{H}), 7.30-7.27$ $(\mathrm{t}, J=7.5 \mathrm{~Hz}, 2 \mathrm{H}), 7.21-7.18(\mathrm{~m}, 3 \mathrm{H}), 5.70-5.68(\mathrm{~d}, J=8.2 \mathrm{~Hz}, 1 \mathrm{H}), 4.71-4.68(\mathrm{dd}, J$ $=7.9,5.9 \mathrm{~Hz}, 1 \mathrm{H}), 2.82-2.70(\mathrm{~m}, 2 \mathrm{H}), 2.47(\mathrm{~s}, 6 \mathrm{H}), 1.95-1.82(\mathrm{~m}, 2 \mathrm{H}), 1.59-1.55(\mathrm{dd}$, $J=13.9,6.8 \mathrm{~Hz}, 1 \mathrm{H}), 1.28-1.22(\mathrm{~d}, J=28.9 \mathrm{~Hz}, 12 \mathrm{H}) ;{ }^{13} \mathrm{C} \mathrm{NMR}\left(126 \mathrm{MHz}, \mathrm{CDCl}_{3}\right) \delta$ 142.0, 140.9, 133.2, 132.8, 128.6, 128.4, 128.4, 127.8, 127.8, 126.3, 125.9, 125.4, 124.7, 84.1, 59.2, 37.7, 35.2, 30.9, 25.0, 24.9; ${ }^{11} \mathrm{~B}$ NMR (161 MHz, $\left.\mathrm{CDCl}_{3}\right) \delta 33.8 ; \mathrm{HRMS}(\mathrm{EI}) \mathrm{m} / \mathrm{z}$ calcd. for $\mathrm{C}_{28} \mathrm{H}_{37} \mathrm{BN}_{2} \mathrm{O}_{4} \mathrm{~S}[\mathrm{M}]^{+}: 508.2567$, found: 508.2565; CHIRALCEL IA, $n$-hexane : $i$ $\mathrm{PrOH}=99: 1,1 \mathrm{~mL} / \mathrm{min}, 25{ }^{\circ} \mathrm{C}, \lambda=220 \mathrm{~nm}$; $\mathrm{t}_{\mathrm{R}} 11.8 \mathrm{~min}$ (major), $\mathrm{t}_{\mathrm{R}} 13.9 \mathrm{~min}$ (minor); Analogous mixtures of enantiomers were prepared using $( \pm)-\mathbf{L} \mathbf{1}$ as a ligand under the standard reaction procedure.

[Racemic 3k]

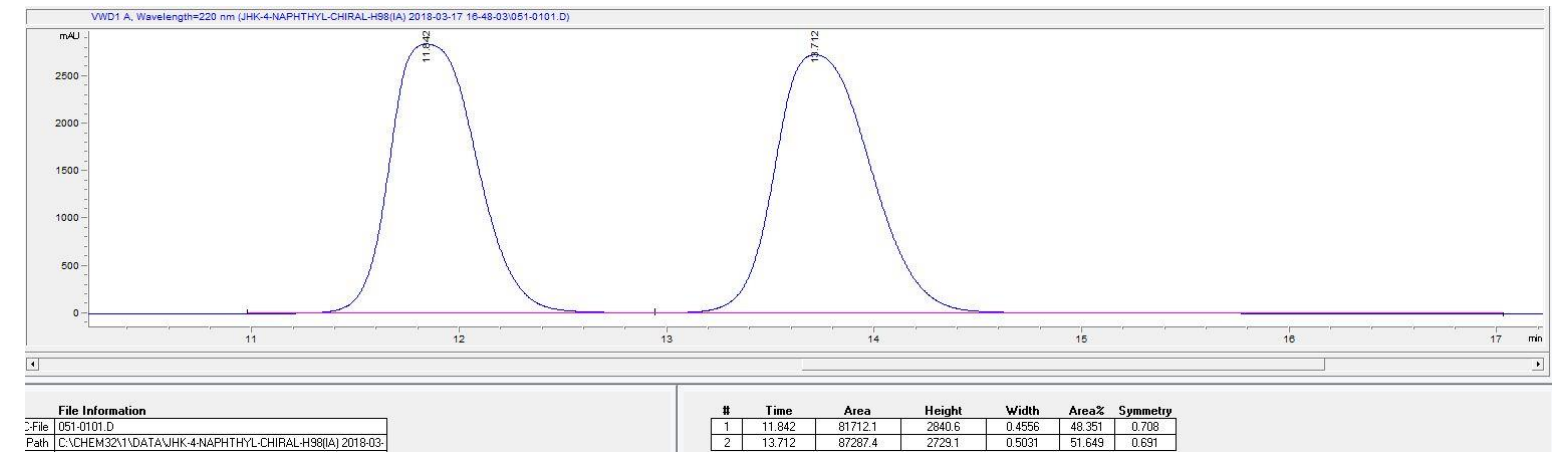

[Enantioenriched 3k]

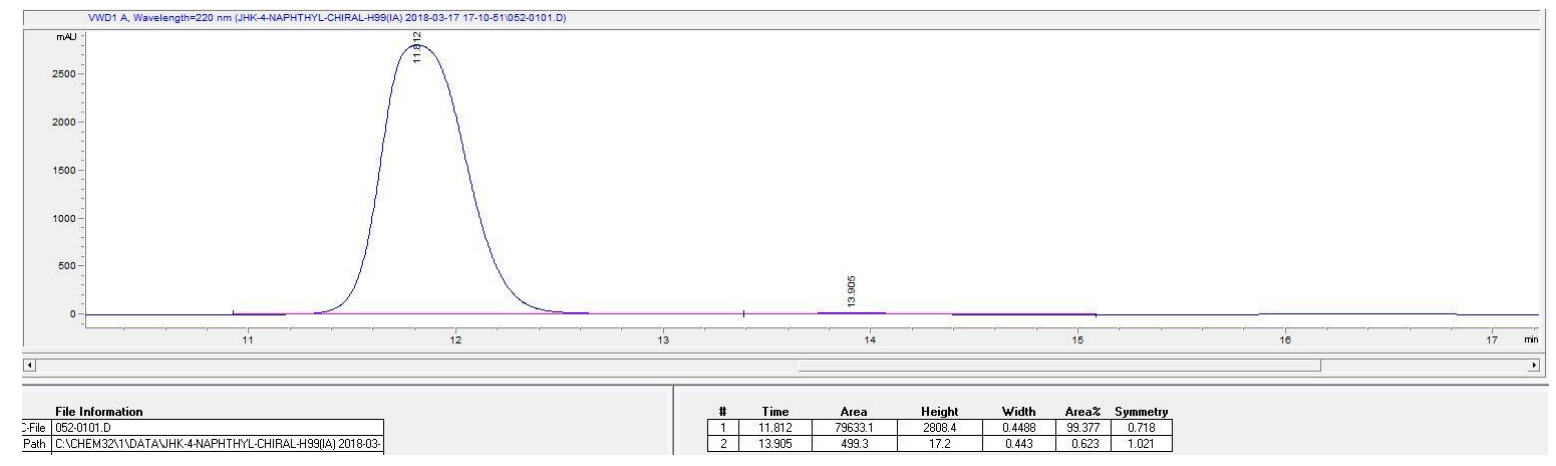


<smiles>CNS(=O)(=O)NC(c1cccs1)C(CCc1ccccc1)c1ccccc1</smiles>

$N, N$-Dimethyl- $N^{\prime}$-[(1S,2R)-4-phenyl-2-(4,4,5,5-tetramethyl-1,3,2-dioxaborolan-2-yl)-1(thiophen-2-yl)butyl]sulfamide (Table 2, 31): CuBr (1.4 mg, $0.010 \mathrm{mmol}, 5.0 \mathrm{~mol} \%$ ), L1 (7.2 mg, $0.020 \mathrm{mmol}, 10 \mathrm{~mol} \%$ ), LiO $t \mathrm{Bu}$ (32 mg, $0.40 \mathrm{mmol}$ ), 1,1-diboryl-3-phenylpropane (112 mg, $0.30 \mathrm{mmol}), N, N$-dimethyl- $N^{\prime}$-[(2-thienyl)methylene)] sulfamide (44 mg, 0.20 mmol) and 1,4-dioxane/toluene $(0.40 \mathrm{~mL}$, v/v $1: 1)$ at $50{ }^{\circ} \mathrm{C}$ for $24 \mathrm{~h} .{ }^{1} \mathrm{H}-\mathrm{NMR}$ analysis of the crude mixture indicated 9:1 diastereoselectivity. The crude mixture was purified by silica gel column chromatography $\left(\mathrm{Et}_{2} \mathrm{O}: n\right.$-hexane $=1: 2$ to $\left.1: 1\right)$ to give the corresponding $\beta$ aminoboronate ester $3 \mathrm{l}$ as a brown solid (49 mg, 53\%, 97\% ee); $[\alpha]_{\mathrm{D}}^{23}+8.3\left(c 0.47, \mathrm{CH}_{2} \mathrm{Cl}_{2}\right)$; Major diastereomer; ${ }^{1} \mathrm{H}$ NMR $\left(500 \mathrm{MHz}, \mathrm{CDCl}_{3}\right) \delta 7.29-7.26(\mathrm{~m}, 2 \mathrm{H}), 7.21-7.16(\mathrm{~m}, 4 \mathrm{H})$, $6.94-6.90(\mathrm{~m}, 2 \mathrm{H}), 5.52-5.51(\mathrm{~d}, J=8.5 \mathrm{~Hz}, 1 \mathrm{H}), 4.82-4.79(\mathrm{dd}, J=8.5,5.4 \mathrm{~Hz}, 1 \mathrm{H})$, $2.79-2.67$ (m, 2H), $2.63(\mathrm{~s}, 6 \mathrm{H}), 1.90-1.85(\mathrm{dd}, J=15.7,7.8 \mathrm{~Hz}, 2 \mathrm{H}), 1.62-1.58$ (dd, $J$ $=12.9,7.4 \mathrm{~Hz}, 1 \mathrm{H}), 1.23-1.22(\mathrm{~d}, J=6.2 \mathrm{~Hz}, 12 \mathrm{H}) ;{ }^{13} \mathrm{C} \mathrm{NMR}\left(126 \mathrm{MHz}, \mathrm{CDCl}_{3}\right) \delta 148.0$, 142.0, 128.6, 128.4, 126.7, 125.9, 124.6, 124.2, 84.1, 55.3, 37.9, 35.1, 30.7, 24.9, 24.9; ${ }^{11} \mathrm{~B}$ NMR (161 MHz, $\left.\mathrm{CDCl}_{3}\right) \delta 33.5$; HRMS (EI) m/z calcd. for $\mathrm{C}_{22} \mathrm{H}_{33} \mathrm{BN}_{2} \mathrm{O}_{4} \mathrm{~S}_{2}[\mathrm{M}]^{+}: 464.1975$, found: 464.1978; CHIRALCEL IB, $n$-hexane : $i$-PrOH $=98: 2,1 \mathrm{~mL} / \mathrm{min}, 25^{\circ} \mathrm{C}, \lambda=220$ $\mathrm{nm} ; \mathrm{t}_{\mathrm{R}} 5.3 \mathrm{~min}$ (minor), $\mathrm{t}_{\mathrm{R}} 6.2 \mathrm{~min}$ (major); Analogous mixtures of enantiomers were prepared using $( \pm)-\mathbf{L} \mathbf{1}$ as a ligand under the standard reaction procedure.

[Racemic 3I]

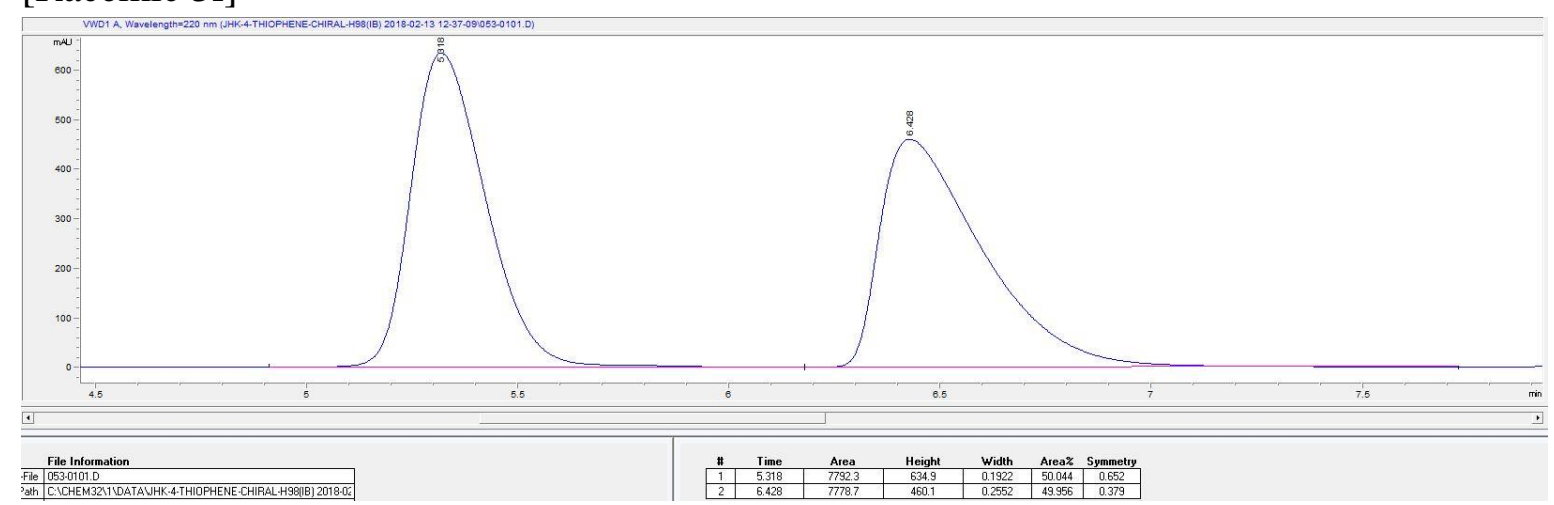

[Enantioenriched 3I]

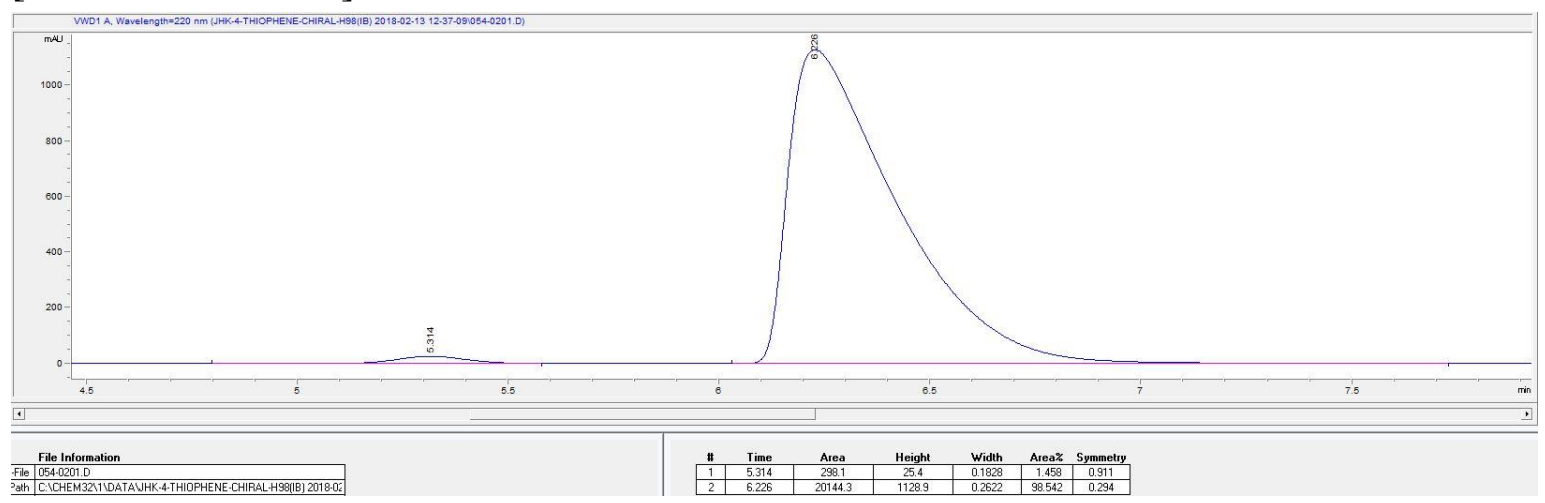


<smiles>CC(c1ccccc1)C(NS(C)(=O)=O)c1ccccc1</smiles>

$N, N$-Dimethyl- $N^{\prime}-N$-[(1S,2R)-1-phenyl-2-(4,4,5,5-tetramethyl-1,3,2-dioxaborolan-2yl)propyl]sulfamide (Table 2, 3m): CuBr (1.4 mg, $0.010 \mathrm{mmol}, 5.0 \mathrm{~mol} \%)$, L2 (7.7 mg, $0.020 \mathrm{mmol}, 10 \mathrm{~mol} \%), \mathrm{LiO} t \mathrm{Bu}(32 \mathrm{mg}, 0.40 \mathrm{mmol}), 1,1$-dibroylethane ( $85 \mathrm{mg}, 0.30 \mathrm{mmol})$, $N, N$-dimethyl- $N$ '-(phenylmethylene)sulfamide (43 mg, $0.20 \mathrm{mmol}$ ) and 1,4-dioxane/toluene $(0.40 \mathrm{~mL}, \mathrm{v} / \mathrm{v} 1: 1)$ at room temperature for $24 \mathrm{~h} .{ }^{1} \mathrm{H}-\mathrm{NMR}$ spectroscopic analysis of the crude mixture indicated $96 \%{ }^{1} \mathrm{H}-\mathrm{NMR}$ yield and 7:1 diastereoselectivity. The crude mixture was purified by silica gel column chromatography $\left(\mathrm{Et}_{2} \mathrm{O}: n\right.$-hexane $=1: 3$ to $\left.1: 1\right)$ to give the corresponding $\beta$-aminoboronate ester $\mathbf{3 m}$ as a white solid (41 $\mathrm{mg}, 55 \%, 91 \%$ ee); Major diastereomer; ${ }^{1} \mathrm{H}$ NMR $\left(500 \mathrm{MHz}, \mathrm{CDCl}_{3}\right) \delta 7.32-7.28(\mathrm{~m}, 4 \mathrm{H}), 7.24-7.20(\mathrm{~m}, 1 \mathrm{H}), 5.46$ $-5.44(\mathrm{~d}, J=7.5 \mathrm{~Hz}, 1 \mathrm{H}), 4.32-4.29(\mathrm{t}, J=7.0 \mathrm{~Hz}, 1 \mathrm{H}), 2.46(\mathrm{~s}, 6 \mathrm{H}), 1.50-1.44(\mathrm{p}, J=$ $7.3 \mathrm{~Hz}, 1 \mathrm{H}), 1.23-1.21(\mathrm{~d}, J=9.6 \mathrm{~Hz}, 12 \mathrm{H}), 1.05-1.04(\mathrm{~d}, J=7.5 \mathrm{~Hz}, 3 \mathrm{H}) ;{ }^{13} \mathrm{C} \mathrm{NMR}(126$ $\left.\mathrm{MHz}, \mathrm{CDCl}_{3}\right) \delta 143.4,128.4,127.4,126.8,83.9,61.4,37.6,24.9,24.8,13.9 ;{ }^{11} \mathrm{~B}$ NMR (161 $\left.\mathrm{MHz}, \mathrm{CDCl}_{3}\right) \delta$ 33.6; HRMS (EI) $\mathrm{m} / \mathrm{z}$ calcd. for $\mathrm{C}_{17} \mathrm{H}_{29} \mathrm{BN}_{2} \mathrm{O}_{4} \mathrm{~S}[\mathrm{M}]^{+}:$368.1941, found: 368.1938; CHIRALCEL IA, $n$-hexane : $i$-PrOH = 98:2, $1 \mathrm{~mL} / \mathrm{min}, 25^{\circ} \mathrm{C}, \lambda=220 \mathrm{~nm}$; $\mathrm{t}_{\mathrm{R}} 3.3$ $\min$ (major), $t_{R} 3.9$ min (minor); Analogous mixtures of enantiomers were prepared using $( \pm)-\mathbf{L} \mathbf{1}$ as a ligand under the standard reaction procedure.

[Racemic 3m]

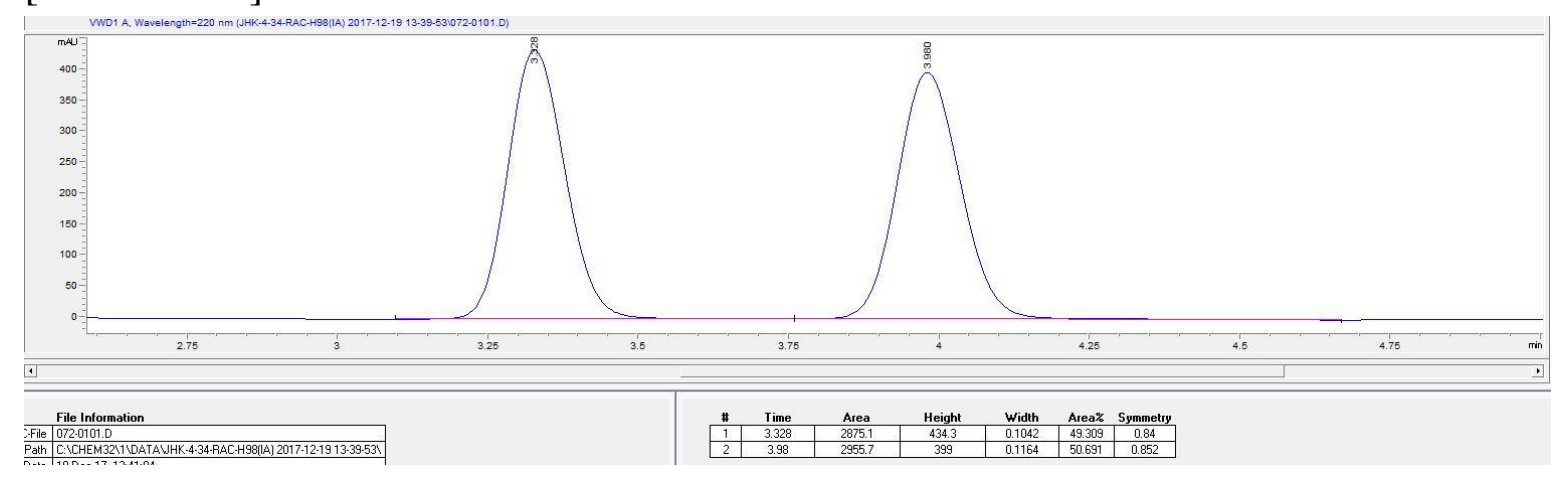

[Enantioenriched 3m]

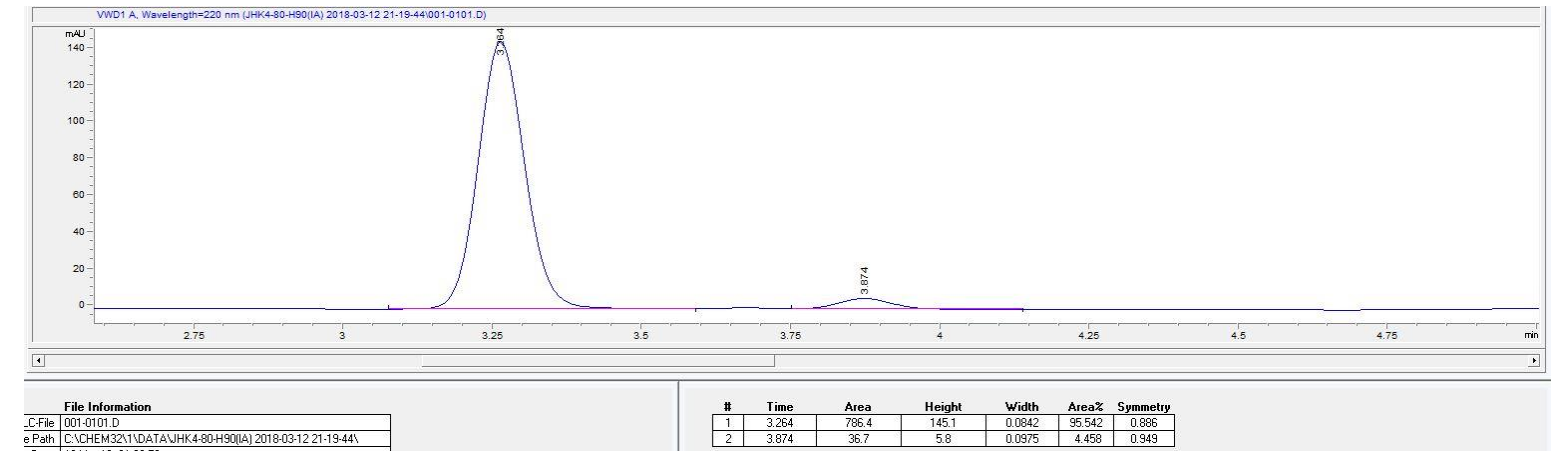


<smiles>CC(O)C(NS(C)(=O)=O)c1ccccc1</smiles>

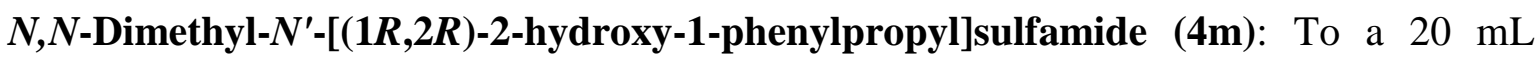
dram vial equipped with a magnetic stir bar were added the above obtained crude $\beta$ aminoboronate ester 3m (ca. $0.20 \mathrm{mmol})$ and $\mathrm{NaHCO}_{3}(101 \mathrm{mg}, 1.2 \mathrm{mmol})$ in $\mathrm{THF} / \mathrm{H}_{2} \mathrm{O}$ $(2.0 \mathrm{~mL}, \mathrm{v} / \mathrm{v} 1: 1)$ at room temperature. To this solution, $\mathrm{H}_{2} \mathrm{O}_{2}(37 \mathrm{wt} \%, 0.50 \mathrm{~mL})$ was slowly added and stirred for $3 \mathrm{~h}$ at room temperature.. The reaction mixture was poured into a separatory funnel and brine $(20 \mathrm{~mL})$ was added. The aqueous phase was extracted with EtOAc (20 mL x 3) and the combined organics were dried over $\mathrm{MgSO}_{4}$, filtered, and concentrated under reduced pressure. ${ }^{1} \mathrm{H}-\mathrm{NMR}$ analysis of the crude mixture indicated 7:1 diastereoselectivity. The crude mixture was purified by silica gel column chromatography (EtOAc : $n$-hexane $=1: 2$ to $1: 1$ ) to give the corresponding $\beta$-amino alcohol $\mathbf{4 m}$ as a yellow solid $\left(47 \mathrm{mg}, 91 \%, 91 \%\right.$ ee); $[\alpha]_{\mathrm{D}}^{23}-19.2\left(c 0.42, \mathrm{CH}_{2} \mathrm{Cl}_{2}\right)$; Major diastereomer; ${ }^{1} \mathrm{H} \mathrm{NMR}$ $\left(500 \mathrm{MHz}, \mathrm{CDCl}_{3}\right) \delta 7.39-7.34(\mathrm{~m}, 2 \mathrm{H}), 7.31-7.27(\mathrm{~m}, 3 \mathrm{H}), 5.64-5.63(\mathrm{~d}, J=6.3 \mathrm{~Hz}$, $1 \mathrm{H}), 4.17-4.15(\mathrm{t}, J=6.5 \mathrm{~Hz}, 1 \mathrm{H}), 3.91-3.86(\mathrm{p}, J=6.3 \mathrm{~Hz}, 1 \mathrm{H}), 2.68(\mathrm{~s}, 1 \mathrm{H}), 2.47$ (s, $6 \mathrm{H}), 1.15-1.14(\mathrm{~d}, J=6.3 \mathrm{~Hz}, 3 \mathrm{H}) ;{ }^{13} \mathrm{C} \mathrm{NMR}\left(126 \mathrm{MHz}, \mathrm{CDCl}_{3}\right) \delta 140.1,128.8,128.1$, 127.4, 71.1, 64.7, 37.6, 20.4; HRMS (EI) m/z calcd. for $\mathrm{C}_{11} \mathrm{H}_{18} \mathrm{~N}_{2} \mathrm{O}_{3} \mathrm{~S}[\mathrm{M}]^{+}:$258.1038, found: 258.1040; CHIRALCEL OD-H, $n$-hexane : $i$-PrOH $=90: 10,1 \mathrm{~mL} / \mathrm{min}, 40^{\circ} \mathrm{C}, \lambda=$ $220 \mathrm{~nm}$; $\mathrm{t}_{\mathrm{R}} 9.4 \mathrm{~min}$ (minor), $\mathrm{t}_{\mathrm{R}} 13.1 \mathrm{~min}$ (major); Analogous mixtures of enantiomers were prepared using $( \pm)-\mathbf{L} \mathbf{1}$ as a ligand under the standard reaction procedure.

[Racemic 4m]

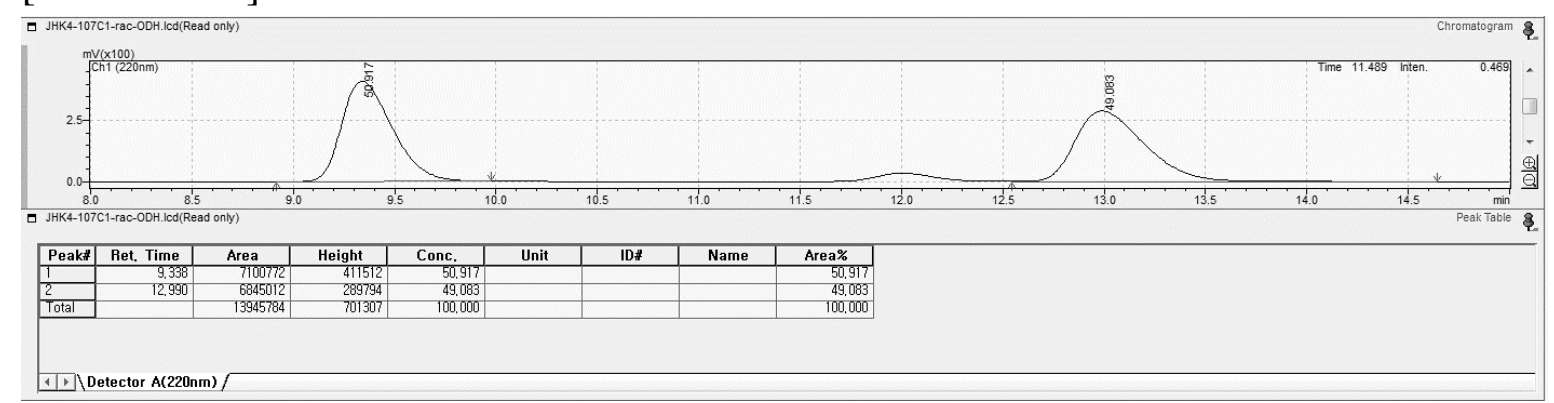

[Enantioenriched 4m]

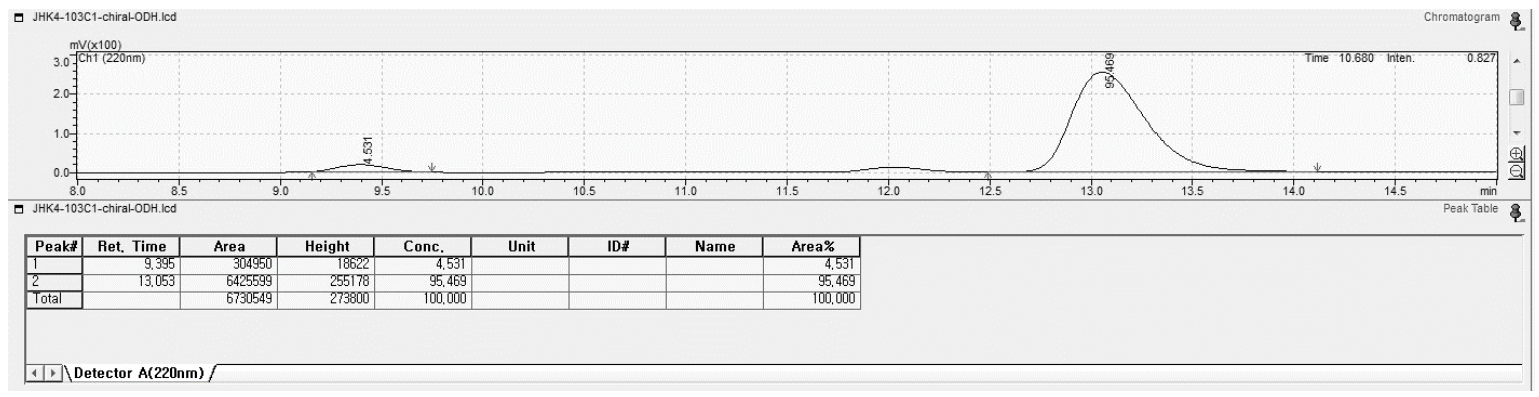


<smiles>COC1(CC[C@H](c2ccccc2)[C@@H](NS(C)(=O)=O)c2ccccc2)OCCO1</smiles>

$N, N$-Dimethyl- $N^{\prime}$-[(1S,2R)-4-(1,3-dioxolan-2-yl)-1-phenyl-2-(4,4,5,5-tetramethyl-1,3,2dioxaborolan-2-yl)butyl]sulfamide (Table 2, 3n): $\mathrm{CuBr}$ (1.4 mg, $0.010 \mathrm{mmol}, 5.0 \mathrm{~mol} \%$ ), L1 (7.2 mg, $0.020 \mathrm{mmol}, 10 \mathrm{~mol} \%)$, LiOtBu (32 mg, $0.40 \mathrm{mmol}), 1$, 1-diboryl-(3-(1,3dioxolan-2-yl)propane (110 mg, $0.30 \mathrm{mmol}), N, N$-dimethyl- $N$ '-(phenylmethylene)sulfamide (43 mg, $0.20 \mathrm{mmol}$ ) and 1,4-dioxane/toluene $\left(0.40 \mathrm{~mL}\right.$, v/v 1:1) at $50{ }^{\circ} \mathrm{C}$ for $24 \mathrm{~h} .{ }^{1} \mathrm{H}-\mathrm{NMR}$ analysis of the crude mixture indicated 10:1 diastereoselectivity. The crude mixture was purified by silica gel column chromatography (EtOAc : $n$-hexane $=1: 2$ ) to give the corresponding $\beta$-aminoboronate ester $3 \mathbf{n}$ as a white solid (55 mg, 60\%, 94\% ee); $[\alpha]_{\mathrm{D}}^{23}-4.3$ (c $0.35, \mathrm{CH}_{2} \mathrm{Cl}_{2}$ ); Major diastereomer; ${ }^{1} \mathrm{H} \mathrm{NMR}\left(500 \mathrm{MHz}, \mathrm{CDCl}_{3}\right) \delta 7.31-7.27(\mathrm{~m}, 4 \mathrm{H})$, $7.23-7.18(\mathrm{~m}, 1 \mathrm{H}), 5.51-5.49(\mathrm{~d}, J=8.2 \mathrm{~Hz}, 1 \mathrm{H}), 4.83-4.81(\mathrm{t}, J=4.6 \mathrm{~Hz}, 1 \mathrm{H}), 4.45-$ $4.42(\mathrm{dd}, J=7.9,5.9 \mathrm{~Hz}, 1 \mathrm{H}), 3.95-3.89(\mathrm{~m}, 2 \mathrm{H}), 3.84-3.78(\mathrm{~m}, 2 \mathrm{H}), 2.48(\mathrm{~s}, 6 \mathrm{H}), 1.80$ $-1.56(\mathrm{~m}, 4 \mathrm{H}), 1.46-1.41(\mathrm{dd}, J=14.4,6.2 \mathrm{~Hz}, 1 \mathrm{H}), 1.22-1.17(\mathrm{~d}, J=20.7 \mathrm{~Hz}, 12 \mathrm{H}) ;{ }^{13} \mathrm{C}$ NMR $\left(126 \mathrm{MHz}, \mathrm{CDCl}_{3}\right) \delta 143.4,128.4,127.3,126.6,104.5,84.0,64.9,64.9,59.4,37.7$, 33.1, 24.9, 24.9, 23.6; ${ }^{11} \mathrm{~B}$ NMR (161 MHz, $\left.\mathrm{CDCl}_{3}\right) \delta 33.7$; HRMS (EI) m/z calcd. for $\mathrm{C}_{21} \mathrm{H}_{35} \mathrm{BN}_{2} \mathrm{O}_{6} \mathrm{~S}[\mathrm{M}]^{+}:$454.2309, found: 454.2310; CHIRALCEL IB, $n$-hexane $: i$-PrOH $=$ $98: 2,1 \mathrm{~mL} / \mathrm{min}, 25^{\circ} \mathrm{C}, \lambda=220 \mathrm{~nm}$; $\mathrm{t}_{\mathrm{R}} 12.1 \mathrm{~min}$ (minor), $\mathrm{t}_{\mathrm{R}} 13.9 \mathrm{~min}$ (major); Analogous mixtures of enantiomers were prepared using $( \pm)-\mathbf{L} \mathbf{1}$ as a ligand under the standard reaction procedure.

\section{[Racemic 3n]}

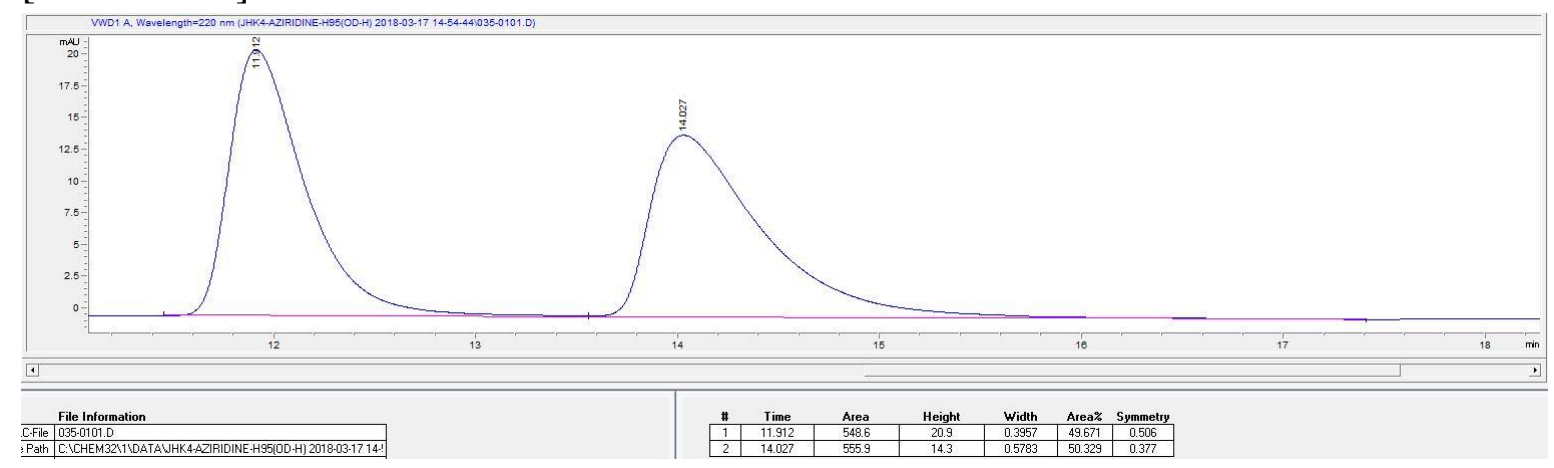

[Enantioenriched 3n]

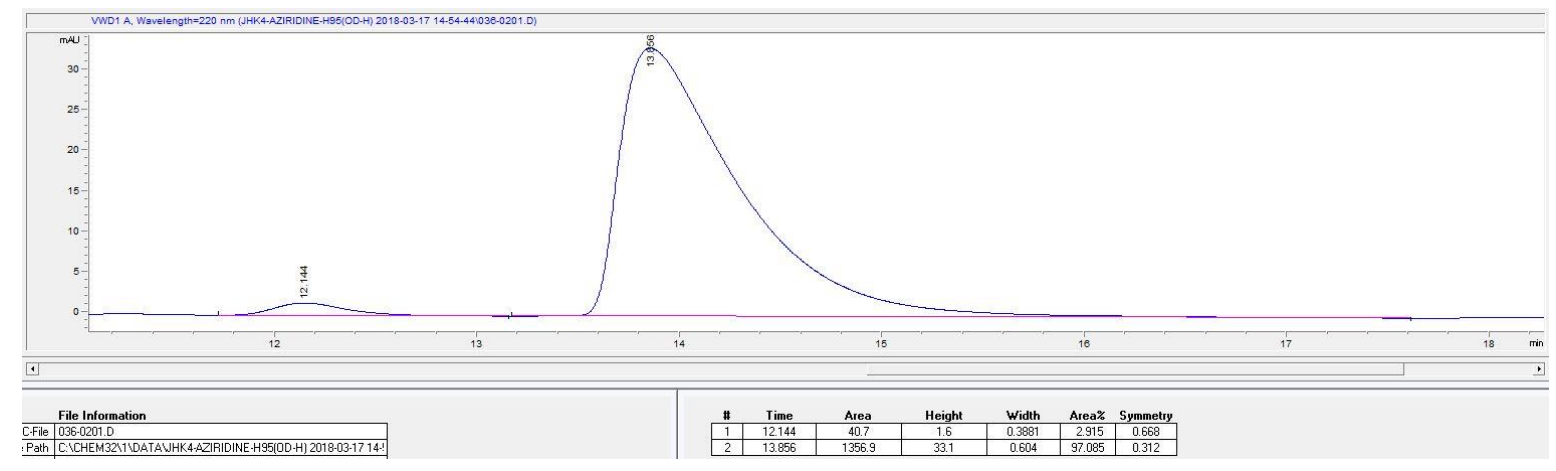


<smiles></smiles>

\section{$N, N$-Dimethyl- $N$ '- $N$ '-[(1S,2R)-4-((tert-butyldimethylsilyl)oxy)-1-phenyl-2-(4,4,5,5-}

tetramethyl-1,3,2-dioxaborolan-2-yl)butyl]sulfamide (Table 2, 3o): $\mathrm{CuBr}(1.4 \mathrm{mg}, 0.010$ mmol, $5.0 \mathrm{~mol} \%$ ), L1 (7.2 mg, $0.020 \mathrm{mmol}, 10 \mathrm{~mol} \%)$, LiOtBu (32 mg, $0.40 \mathrm{mmol}), 1,1-$ diboryl-(3-((tert-butyldimethylsilyl)oxy)propane $(128 \mathrm{mg}, 0.30 \mathrm{mmol}), N, N$-dimethyl- $N^{\prime}-$ (phenylmethylene)sulfamide $(43 \mathrm{mg}, 0.20 \mathrm{mmol})$ and 1,4-dioxane/toluene $(0.40 \mathrm{~mL}, \mathrm{v} / \mathrm{v}$ $1: 1)$ at $50{ }^{\circ} \mathrm{C}$ for $24 \mathrm{~h} .{ }^{1} \mathrm{H}-\mathrm{NMR}$ spectroscopic analysis of the crude mixture indicated $54 \%$ ${ }^{1} \mathrm{H}-\mathrm{NMR}$ yield and 12:1 diastereoselectivity. The title $\beta$-aminoboronate ester $\mathbf{3 o}$ was be purified by silica gel column chromatography (EtOAc : $n$-hexane $=1: 5$ ) to give the corresponding $\beta$-aminoboronate ester 3o as a white solid (25 $\mathrm{mg}, 25 \%, 96 \%$ ee); Major diastereomer; ${ }^{1} \mathrm{H}$ NMR $\left(500 \mathrm{MHz}, \mathrm{CDCl}_{3}\right) \delta 7.30-7.29(\mathrm{~d}, J=4.4 \mathrm{~Hz}, 4 \mathrm{H}), 7.24-7.20(\mathrm{~m}$, $1 \mathrm{H}), 5.55-5.53(\mathrm{~d}, J=8.1 \mathrm{~Hz}, 1 \mathrm{H}), 4.45-4.43(\mathrm{dd}, J=8.0,6.0 \mathrm{~Hz}, 1 \mathrm{H}), 3.74-3.70(\mathrm{~m}$, $1 \mathrm{H}), 3.61-3.55$ (ddd, $J=10.8,8.5,5.4 \mathrm{~Hz}, 1 \mathrm{H}), 2.47$ (s, 6H), $1.75-1.63(\mathrm{~m}, 2 \mathrm{H}), 1.62-$ $1.57(\mathrm{~m}, 2 \mathrm{H}), 1.22-1.19(\mathrm{~d}, J=13.9 \mathrm{~Hz}, 12 \mathrm{H}), 0.88(\mathrm{~s}, 9 \mathrm{H}), 0.03(\mathrm{~s}, 6 \mathrm{H}) ;{ }^{13} \mathrm{C} \mathrm{NMR}(126$ $\left.\mathrm{MHz}, \mathrm{CDCl}_{3}\right) \delta 143.5,128.4,127.3,126.8,84.0,62.0,59.1,37.7,31.8,26.1,25.0,24.9,18.5$, -5.1, -5.2; ${ }^{11} \mathrm{~B}$ NMR (161 MHz, $\mathrm{CDCl}_{3}$ ) $\delta 33.3$; HRMS (EI) m/z calcd. for $\mathrm{C}_{24} \mathrm{H}_{45} \mathrm{BN}_{2} \mathrm{O}_{5} \mathrm{SSi}$ $[\mathrm{M}]^{+}:$512.2912, found: 512.2910; CHIRALCEL IA, $n$-hexane $: i$-PrOH $=99: 1,1 \mathrm{~mL} / \mathrm{min}$, $25{ }^{\circ} \mathrm{C}, \lambda=220 \mathrm{~nm} ; \mathrm{t}_{\mathrm{R}} 4.4 \mathrm{~min}$ (major), $\mathrm{t}_{\mathrm{R}} 5.0 \mathrm{~min}$ (minor); Analogous mixtures of enantiomers were prepared using $( \pm)-\mathbf{L} \mathbf{1}$ as a ligand under the standard reaction procedure.

[Racemic 3o]

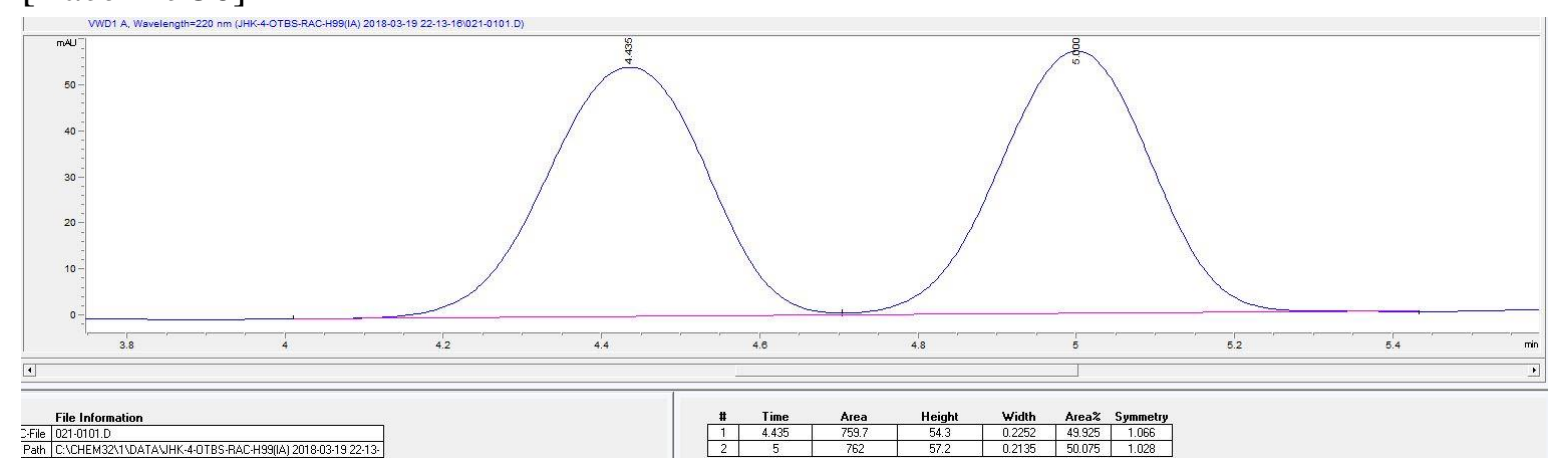

[Enantioenriched 3o]

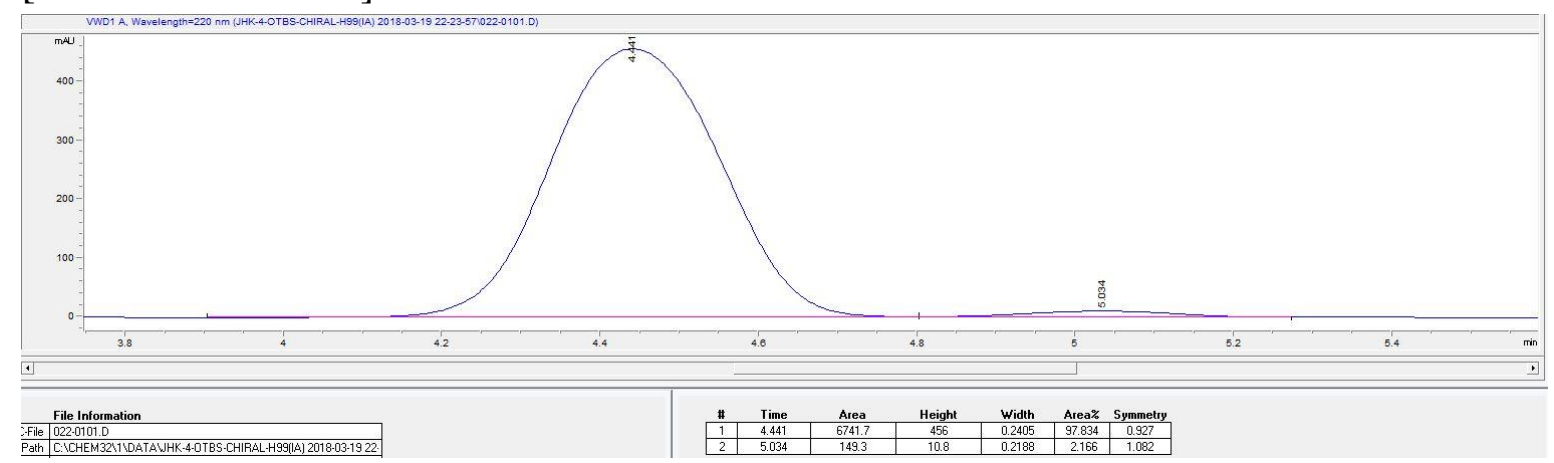


<smiles></smiles>

\section{$N, N$-Dimethyl- $N^{\prime}-[(1 R, 2 R)-4-(($ tert-butyldimethylsilyl)oxy)-2-hydroxy-1-phenylbutyl]}

sulfamide (4o): To a $20 \mathrm{~mL}$ dram vial equipped with a magnetic stir bar were added the above obtained crude $\beta$-aminoboronate ester 30 ( ca. $0.20 \mathrm{mmol}$ ) and $\mathrm{NaHCO}_{3}(101 \mathrm{mg}, 1.2$ $\mathrm{mmol})$ in $\mathrm{THF} / \mathrm{H}_{2} \mathrm{O}(2.0 \mathrm{~mL}, \mathrm{v} / \mathrm{v} 1: 1)$ at room temperature. To this solution, $\mathrm{H}_{2} \mathrm{O}_{2}(37 \mathrm{wt} \%$, $0.50 \mathrm{~mL}$ ) was slowly added and stirred for $3 \mathrm{~h}$ at room temperature. The reaction mixture was poured into a separatory funnel and brine $(20 \mathrm{~mL})$ was added. The aqueous phase was extracted with EtOAc (20 mL x 3) and the combined organics were dried over $\mathrm{MgSO}_{4}$, filtered, and concentrated under reduced pressure. ${ }^{1} \mathrm{H}-\mathrm{NMR}$ analysis of the crude mixture indicated 12:1 diastereoselectivity. The crude mixture was purified by silica gel column chromatography (EtOAc : $n$-hexane $=1: 5$ ) to give the corresponding $\beta$-amino alcohol 4o as a white solid (38 mg, 47\%, 96\% ee); $[\alpha]_{\mathrm{D}}^{23}-13.7\left(c 0.36, \mathrm{CH}_{2} \mathrm{Cl}_{2}\right)$; Major diastereomer; ${ }^{1} \mathrm{H}$ NMR $\left(500 \mathrm{MHz}, \mathrm{CDCl}_{3}\right) \delta 7.36-7.32(\mathrm{~m}, 4 \mathrm{H}), 7.29-7.26(\mathrm{~m}, 1 \mathrm{H}), 5.44-5.43(\mathrm{~d}, J=$ $5.1 \mathrm{~Hz}, 1 \mathrm{H}), 4.27-4.25(\mathrm{t}, J=5.4 \mathrm{~Hz}, 1 \mathrm{H}), 4.01(\mathrm{~s}, 1 \mathrm{H}), 3.96-3.93(\mathrm{ddd}, J=9.2,5.7,2.3$ $\mathrm{Hz}, 1 \mathrm{H}), 3.92-3.88(\mathrm{dt}, J=10.0,4.5 \mathrm{~Hz}, 1 \mathrm{H}), 3.79-3.74(\mathrm{~m}, 1 \mathrm{H}), 2.45(\mathrm{~s}, 6 \mathrm{H}), 1.85-1.77$ $(\mathrm{m}, 1 \mathrm{H}), 1.64-1.59(\mathrm{~m}, 1 \mathrm{H}), 0.88(\mathrm{~s}, 9 \mathrm{H}), 0.07-0.05(\mathrm{~d}, J=7.9 \mathrm{~Hz}, 6 \mathrm{H}) ;{ }^{13} \mathrm{C}$ NMR $(126$ $\left.\mathrm{MHz}, \mathrm{CDCl}_{3}\right) \delta 140.3,128.6,128.0,127.7,75.4,62.9,62.7,37.5,35.0,25.9,18.2,-5.4$, 5.5; HRMS (EI) $\mathrm{m} / \mathrm{z}$ calcd. for $\mathrm{C}_{18} \mathrm{H}_{34} \mathrm{~N}_{2} \mathrm{O}_{4} \mathrm{SSi}[\mathrm{M}]^{+}:$402.2009, found: 402.2011; CHIRALPAK AD-H, $n$-hexane $: i$-PrOH $=90: 10,1 \mathrm{~mL} / \mathrm{min}, 40{ }^{\circ} \mathrm{C}, \lambda=220 \mathrm{~nm} ; \mathrm{t}_{\mathrm{R}} 6.7 \mathrm{~min}$ (major), $\mathrm{t}_{\mathrm{R}} 9.1 \mathrm{~min}$ (minor); Analogous mixtures of enantiomers were prepared using $( \pm)-\mathbf{L} \mathbf{1}$ as a ligand under the standard reaction procedure.

[Racemic 4o]

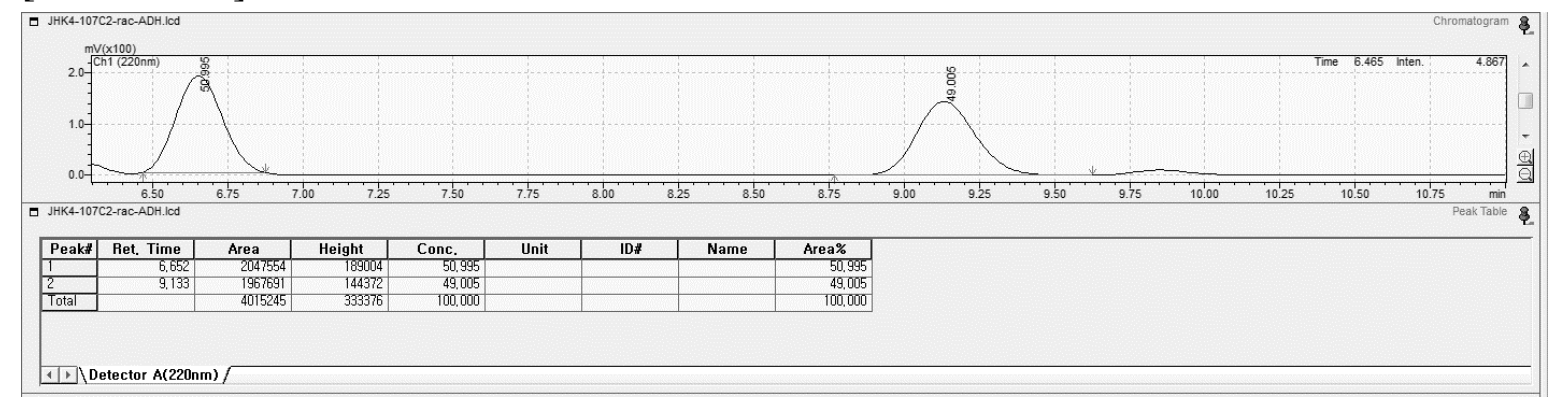

[Enantioenriched 4o]

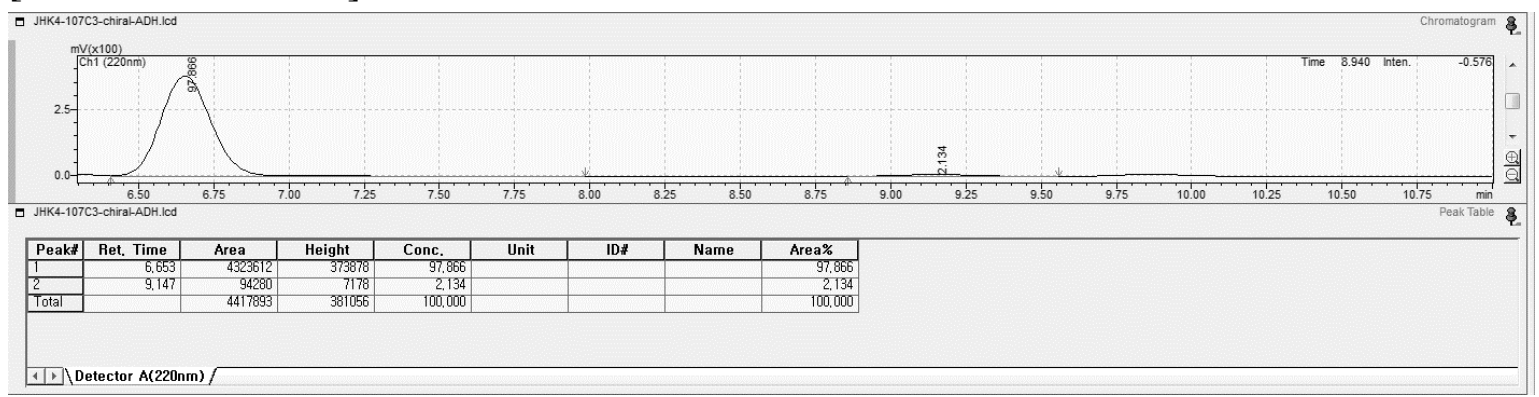




\section{General procedure for synthetic applications}

\subsection{Procedure for the scale-up reaction}

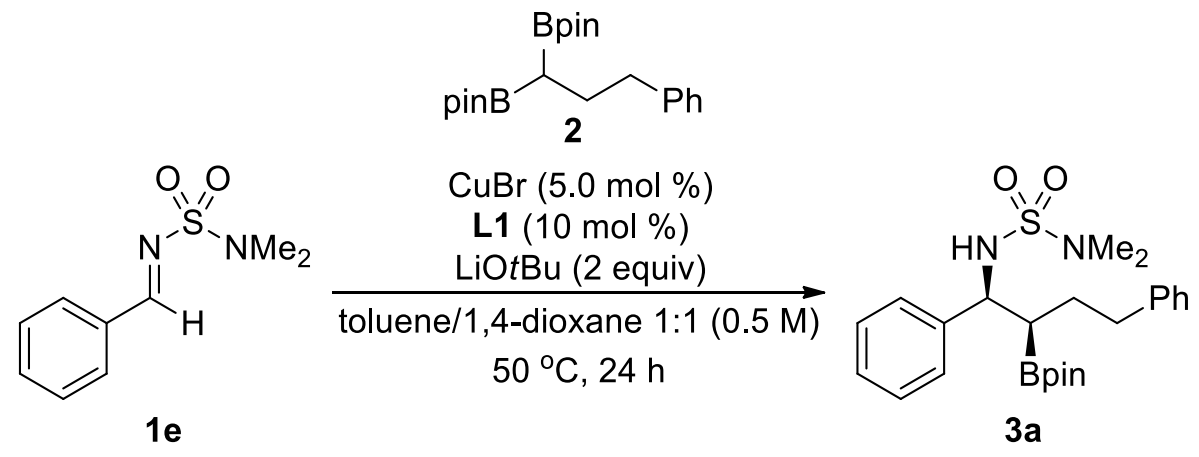

To a $100 \mathrm{~mL}$ round bottomed flask equipped with a magnetic stir bar was added $\mathrm{CuBr}(72$ mg, $0.50 \mathrm{mmol}, 5.0 \mathrm{~mol} \%$ ), L1 (359 mg, $1.00 \mathrm{mmol}, 10 \mathrm{~mol} \%)$, LiOtBu (1.60 g, 20.0 mmol), 1,1-diboryl-3-phenylpropane (5.58 g, $15.0 \mathrm{mmol})$ under air. After sealing with a rubber septum, the flask was evacuated and backfilled with argon three times. To this flask, anhydrous toluene $(7.0 \mathrm{~mL})$ and 1,4-dioxane $(7.0 \mathrm{~mL})$ were added and stirred at room temperature for $1 \mathrm{~h}$. To this mixture, $N, N$-dimethyl- $N$ '-(phenylmethylene)sulfamide $(2.12 \mathrm{~g}$, $10.0 \mathrm{mmol})$ in anhydrous toluene $(3.0 \mathrm{~mL})$ and 1,4-dioxane $(3.0 \mathrm{~mL})$ was added and stirred at $50{ }^{\circ} \mathrm{C}$ for $24 \mathrm{~h}$ under argon atmosphere. The reaction mixture was poured into a separatory funnel and saturated aqueous $\mathrm{NH}_{4} \mathrm{Cl}$ solution $(50 \mathrm{~mL})$ was added. The aqueous phase was extracted with $\mathrm{CH}_{2} \mathrm{Cl}_{2}(50 \mathrm{~mL}$ x 4$)$ and the combined organic layers were dried over $\mathrm{MgSO}_{4}$, filtered, and concentrated under reduced pressure. ${ }^{1} \mathrm{H}-\mathrm{NMR}$ analysis of the crude mixture indicated 13:1 diastereoselectivity. The crude mixture was purified by silica gel column chromatography $\left(\mathrm{Et}_{2} \mathrm{O}: n\right.$-hexane $=1: 3$ to $\left.1: 1\right)$ to give the corresponding $\beta$-aminoboronate ester 3a as a white solid (3.24 g, 71\%, 13:1 d.r., 97\% ee). Recrystallization from $\mathrm{Et}_{2} \mathrm{O} / n$ hexane gave enantiopure 3a $(2.84 \mathrm{~g}, 62 \%,>20: 1$ d.r., >99\% ee).

\subsection{Procedure for the stereospecific vinylation reaction}

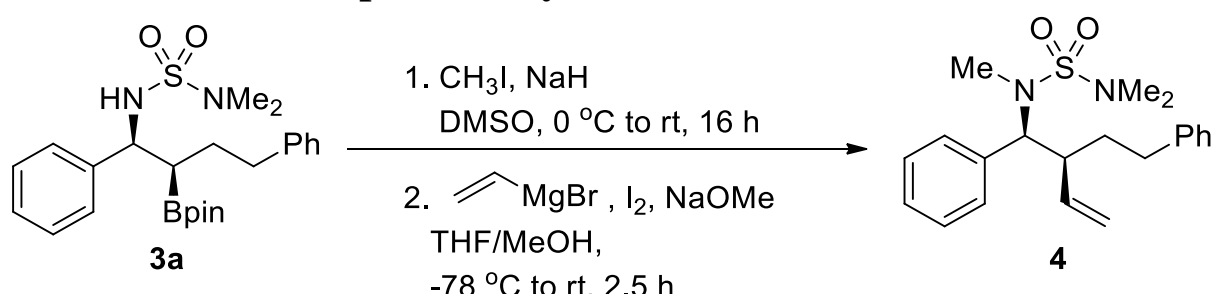

\section{$N, N$-Dimethyl- $N$ '-((1S,2R)-1,4-diphenyl-2-(4,4,5,5-tetramethyl-1,3,2-dioxaborolan-2-} yl)butyl)- $N$ '-methylsulfamide ( $N$-methylated 3a): To an oven-dried $100 \mathrm{~mL}$ round bottomed flask equipped with a magnetic stir bar was added $\mathrm{NaH}$ (60\% in mineral oil, 144 $\mathrm{mg}, 3.60 \mathrm{mmol})$ in DMSO $(3.0 \mathrm{~mL})$ at $0{ }^{\circ} \mathrm{C}$ under argon. To this solution, $\beta$-aminoboronate ester 3a (1.38 g, $3.00 \mathrm{mmol}$, >20:1 d.r., >99\% ee) in DMSO (12 mL) was added dropwise and stirred for $30 \mathrm{~min}$ at room temperature. Then, methyl iodide $(0.280 \mathrm{~mL}, 4.50 \mathrm{mmol})$ was added in one portion and the mixture was stirred at room temperature for $16 \mathrm{~h}$. The reaction mixture was poured in separatory funnel, diluted with EtOAc $(50 \mathrm{~mL})$, and washed with brine $(50 \mathrm{~mL}$ x 3$)$. The combined organic layers were dried over $\mathrm{MgSO}_{4}$ and concentrated under reduced pressure. ${ }^{1} \mathrm{H}-\mathrm{NMR}$ analysis of the crude mixture indicated >20:1 
diastereoselectivity. The crude mixture was purified by silica gel column chromatography $\left(\mathrm{Et}_{2} \mathrm{O}: n\right.$-hexane $\left.=1: 3\right)$ to give $N$-methylated 3a as a white solid $(1.16 \mathrm{~g}, 82 \%) ;{ }^{1} \mathrm{H} \mathrm{NMR}$ $\left(500 \mathrm{MHz}, \mathrm{CDCl}_{3}\right) \delta 7.31-7.23(\mathrm{~m}, 5 \mathrm{H}), 7.22-7.19(\mathrm{t}, J=7.4 \mathrm{~Hz}, 2 \mathrm{H}), 7.14-7.11(\mathrm{t}, J=$ $7.3 \mathrm{~Hz}, 1 \mathrm{H}), 7.02-7.01(\mathrm{~d}, J=7.3 \mathrm{~Hz}, 2 \mathrm{H}), 4.92-4.90(\mathrm{~d}, J=12.3 \mathrm{~Hz}, 1 \mathrm{H}), 2.85(\mathrm{~s}, 3 \mathrm{H})$, $2.67-2.61(\mathrm{ddd}, J=14.6,11.0,4.6 \mathrm{~Hz}, 1 \mathrm{H}), 2.45-2.41(\mathrm{~m}, 1 \mathrm{H}), 2.39(\mathrm{~s}, 6 \mathrm{H}), 1.98-1.92$ $(\mathrm{td}, J=11.8,3.5 \mathrm{~Hz}, 1 \mathrm{H}), 1.74-1.67(\mathrm{~m}, 1 \mathrm{H}), 1.38-1.36(\mathrm{~d}, J=8.4 \mathrm{~Hz}, 12 \mathrm{H}), 1.27-1.20$ $(\mathrm{m}, 1 \mathrm{H}) ;{ }^{13} \mathrm{C} \mathrm{NMR}\left(126 \mathrm{MHz}, \mathrm{CDCl}_{3}\right) \delta 142.2,138.3,128.6,128.5,128.4,128.3,128.0$, 125.9, 84.0, 63.2, 37.9, 35.5, 31.2, 30.6, 25.8, 24.8; ${ }^{11} \mathrm{~B} \mathrm{NMR}\left(161 \mathrm{MHz}, \mathrm{CDCl}_{3}\right) \delta 33.3$; HRMS (EI) m/z calcd. for $\mathrm{C}_{25} \mathrm{H}_{37} \mathrm{BN}_{2} \mathrm{O}_{4} \mathrm{~S}[\mathrm{M}]^{+}$: 472.2567, found: 472.2565.

$N, N$-Dimethyl- $N$ '-[(1S,2S)-2-phenethyl-1-phenylbut-3-en-1-yl]sulfamide (Scheme 3, 4): The stereospecific vinylation was conducted according to a literature procedure. ${ }^{8}$ To an oven-dried $250 \mathrm{~mL}$ round bottomed flask equipped with a magnetic stir bar was added the above obtained $N$-methylated 3a (1.16 g, $2.46 \mathrm{mmol}$, >20:1 d.r.) in THF (24 mL). To this solution, vinylmagnesium bromide $(9.84 \mathrm{~mL}, 9.84 \mathrm{mmol}, 1.0 \mathrm{M}$ in THF) was added dropwise at room temperature and stirred at room temperature for $30 \mathrm{~min}$. Then, the reaction mixture was cooled to $-78{ }^{\circ} \mathrm{C}$ and $\mathrm{I}_{2}(2.50 \mathrm{~g}, 9.84 \mathrm{mmol})$ in $\mathrm{MeOH}(36 \mathrm{~mL})$ was added dropwise. After stirring for $30 \mathrm{~min}$ at $-78{ }^{\circ} \mathrm{C}$, a solution of $\mathrm{NaOMe}(1.06 \mathrm{~g}, 19.7 \mathrm{mmol})$ in $\mathrm{MeOH}(36 \mathrm{~mL})$ was added. The reaction mixture was warmed to room temperature and stirred for additional $1.5 \mathrm{~h}$. To this solution, $n$-hexane $(50 \mathrm{~mL})$ and an aqueous solution of $\mathrm{Na}_{2} \mathrm{~S}_{2} \mathrm{O}_{3}(10 \%, 80 \mathrm{~mL})$ were sequentially added. The reaction mixture was poured in separatory funnel, the aqueous phase was extracted with EtOAc $(80 \mathrm{~mL} \times 3)$. The combined organic layers were dried over $\mathrm{MgSO}_{4}$ and concentrated under reduced pressure. ${ }^{1} \mathrm{H}-\mathrm{NMR}$ analysis of the crude mixture indicated $>20: 1$ diastereoselectivity. The crude mixture was purified by silica gel column chromatography (EtOAc : $n$-hexane $=1: 5$ ) to give the corresponding homoallylic amine 4 as a colorless oil (605 mg, 66\%, >20:1 d.r., >99\% ee); $[\alpha]_{\mathrm{D}}^{23}-6.0\left(c 0.40, \mathrm{CH}_{2} \mathrm{Cl}_{2}\right) ;{ }^{1} \mathrm{H}$ NMR $\left(500 \mathrm{MHz}, \mathrm{CDCl}_{3}\right) \delta 7.33-7.27$ (m, 3H), $7.24-7.20$ $(\mathrm{m}, 4 \mathrm{H}), 7.17-7.14(\mathrm{t}, J=7.3 \mathrm{~Hz}, 1 \mathrm{H}), 7.01-6.99(\mathrm{~d}, J=7.2 \mathrm{~Hz}, 2 \mathrm{H}), 5.89-5.82(\mathrm{dt}, J=$ $17.0,9.9 \mathrm{~Hz}, 1 \mathrm{H}), 5.28-5.26(\mathrm{dd}, J=10.1,1.6 \mathrm{~Hz}, 1 \mathrm{H}), 5.23-5.19(\mathrm{dd}, J=17.0,1.4 \mathrm{~Hz}$, $1 \mathrm{H}), 4.82-4.80(\mathrm{~d}, J=11.5 \mathrm{~Hz}, 1 \mathrm{H}), 2.77-2.67(\mathrm{~m}, 1 \mathrm{H}), 2.62(\mathrm{~s}, 6 \mathrm{H}), 2.54(\mathrm{~s}, 3 \mathrm{H}), 2.47-$ $2.41(\mathrm{dt}, J=13.9,8.5 \mathrm{~Hz}, 1 \mathrm{H}), 1.51-1.44(\mathrm{~m}, 2 \mathrm{H}), 1.43-1.35(\mathrm{~m}, 1 \mathrm{H}) ;{ }^{13} \mathrm{C} \mathrm{NMR}(126$ $\left.\mathrm{MHz}, \mathrm{CDCl}_{3}\right) \delta 141.8,139.7,136.9,128.9,128.7,128.6,128.4,128.2,125.9,117.4,64.4$, 44.4, 38.1, 33.2, 33.0, 29.3; HRMS (EI) m/z calcd. for $\mathrm{C}_{21} \mathrm{H}_{28} \mathrm{~N}_{2} \mathrm{O}_{2} \mathrm{~S}[\mathrm{M}]^{+}:$372.1871, found: 372.1872; CHIRALPAK AD-H, $n$-hexane : $i$-PrOH $=90: 10,0.5 \mathrm{~mL} / \mathrm{min}, 25^{\circ} \mathrm{C}, \lambda=220$ $\mathrm{nm} ; \mathrm{t}_{\mathrm{R}} 6.3 \mathrm{~min}$ (major), $\mathrm{t}_{\mathrm{R}} 6.7 \mathrm{~min}$ (minor); Analogous mixtures of enantiomers were prepared using racemic $( \pm)-\mathbf{3 a}$ as a substrate under the standard reaction procedure. 
[Racemic 4]

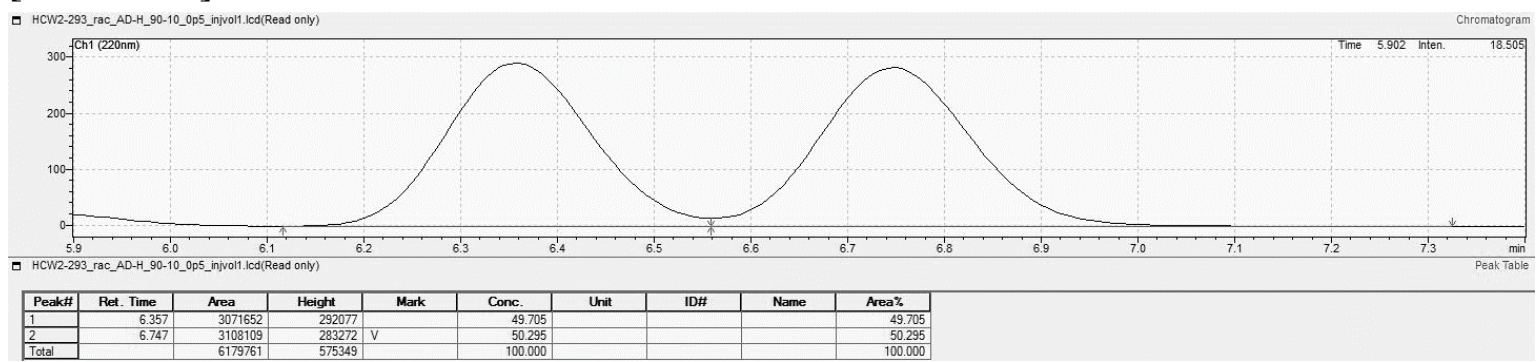

[Enantioenriched 4]

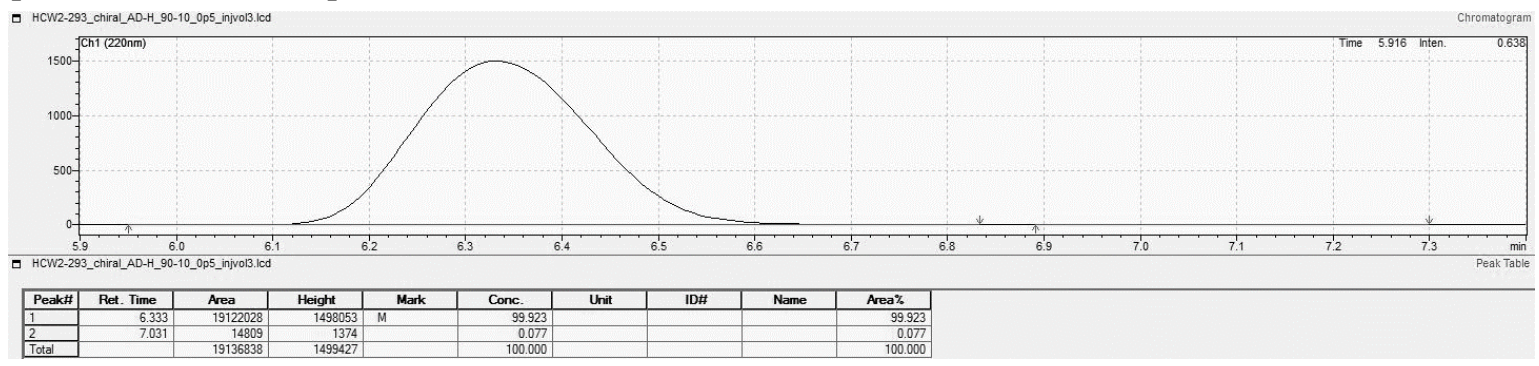

4.3 Procedure for the oxidation of Bpin

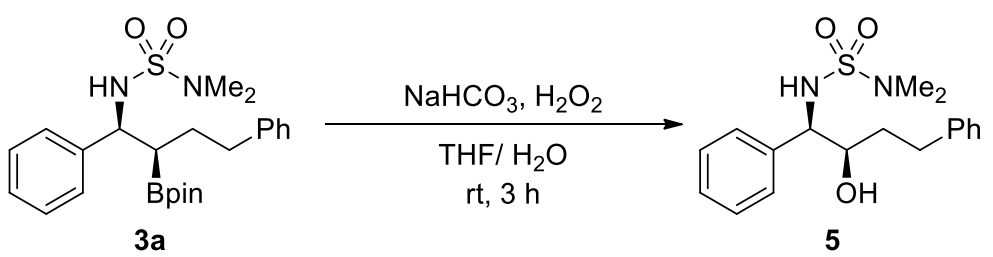

$N, N$-Dimethyl- $N$ '-[(1R,2R)-2-hydroxy-1,4-diphenylbuty]lsulfamide (Scheme 3, 5): To a $100 \mathrm{~mL}$ round bottomed flask with a magnetic stir bar were added enantiopure $\beta$ aminoboronate ester 3a (1.38 g, $3.00 \mathrm{mmol}$, > 20:1 d.r., >99\% ee) and $\mathrm{NaHCO}_{3}(1.51 \mathrm{~g}, 18.0$ $\mathrm{mmol})$ in $\mathrm{THF} / \mathrm{H}_{2} \mathrm{O}(30 \mathrm{~mL}, \mathrm{v} / \mathrm{v} 1: 1)$ at room temperature. To this solution, $\mathrm{H}_{2} \mathrm{O}_{2}$ (37 wt $\%$, $7.5 \mathrm{~mL}$ ) was slowly added and stirred at room temperature for $3 \mathrm{~h}$. The reaction mixture was poured into a separatory funnel and brine $(50 \mathrm{~mL})$ was added. The aqueous phase was extracted with EtOAc ( $50 \mathrm{~mL}$ x 3 ) and the combined organic layers were dried over $\mathrm{MgSO}_{4}$, filtered, and concentrated under reduced pressure. ${ }^{1} \mathrm{H}-\mathrm{NMR}$ analysis of the crude mixture indicated $>20: 1$ diastereoselectivity. The crude mixture was purified by silica gel column chromatography (EtOAc $: n$-hexane $=1: 2$ to $1: 1$ ) to give the corresponding $\beta$-amino alcohol 5 as a white solid (972 mg, 93\%, >20:1 d.r., >99\% ee); $[\alpha]_{\mathrm{D}}^{23}+15.2\left(c 0.46, \mathrm{CH}_{2} \mathrm{Cl}_{2}\right)$; ${ }^{1} \mathrm{H}$ NMR $\left(500 \mathrm{MHz}, \mathrm{CDCl}_{3}\right) \delta 7.37-7.34(\mathrm{t}, J=7.3 \mathrm{~Hz}, 2 \mathrm{H}), 7.31-7.29(\mathrm{~m}, 1 \mathrm{H}), 7.27-$ $7.23(\mathrm{~m}, 4 \mathrm{H}), 7.18-7.17(\mathrm{t}, J=7.3 \mathrm{~Hz}, 1 \mathrm{H}), 7.13-7.12(\mathrm{~d}, J=7.2 \mathrm{~Hz}, 2 \mathrm{H}), 5.46(\mathrm{br} \mathrm{s}, 1 \mathrm{H})$, $4.30-4.29(\mathrm{~d}, J=5.6 \mathrm{~Hz}, 1 \mathrm{H}), 3.73-3.69(\mathrm{dt}, J=8.4,5.0 \mathrm{~Hz}, 1 \mathrm{H}), 2.85-2.79(\mathrm{~m}, 1 \mathrm{H})$, $2.70-2.63(\mathrm{~m}, 1 \mathrm{H}), 2.49(\mathrm{~s}, 6 \mathrm{H}), 2.24$ (br s, $1 \mathrm{H}), 1.87-1.76(\mathrm{~m}, 2 \mathrm{H}) ;{ }^{13} \mathrm{C} \mathrm{NMR}(126 \mathrm{MHz}$, $\left.\mathrm{CDCl}_{3}\right) \delta 141.6,140.1,128.8,128.5,128.5,128.1,127.4,126.0,74.3,63.0,37.6,35.4,31.9$; HRMS (EI) m/z calcd. for $\mathrm{C}_{18} \mathrm{H}_{24} \mathrm{~N}_{2} \mathrm{O}_{3} \mathrm{~S}[\mathrm{M}]^{+}: 348.1508$, found: 348.1507 ; CHIRALPAK AD-H, $n$-hexane $: i$-PrOH =90:10, $1 \mathrm{~mL} / \mathrm{min}, 25^{\circ} \mathrm{C}, \lambda=220 \mathrm{~nm} ; \mathrm{t}_{\mathrm{R}} 10.4 \mathrm{~min}$ (major), $\mathrm{t}_{\mathrm{R}}$ $12.0 \mathrm{~min}$ (minor); Analogous mixtures of enantiomers were prepared using racemic $( \pm)-\mathbf{3 a}$ as a substrate under the standard reaction procedure. 
[Racemic 5]
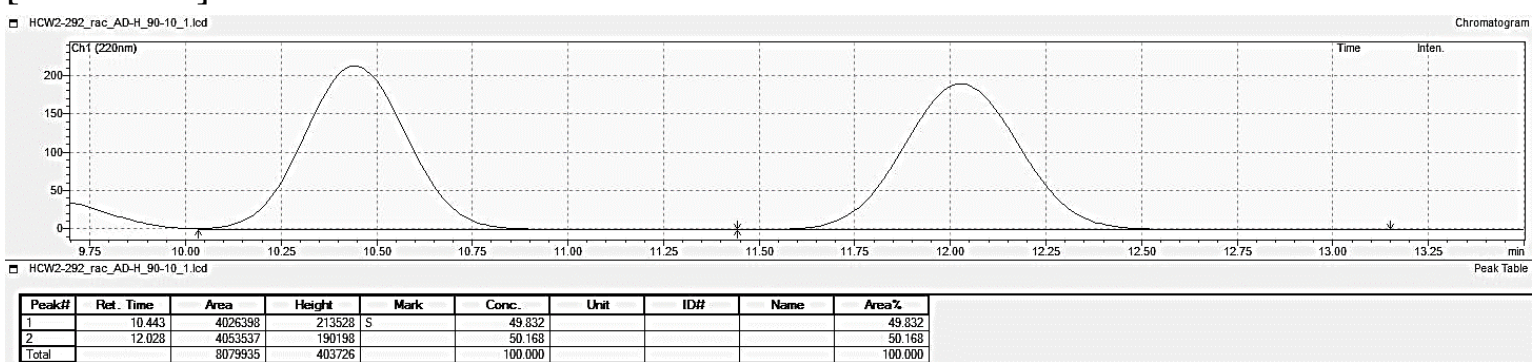

[Enantioenriched 5]

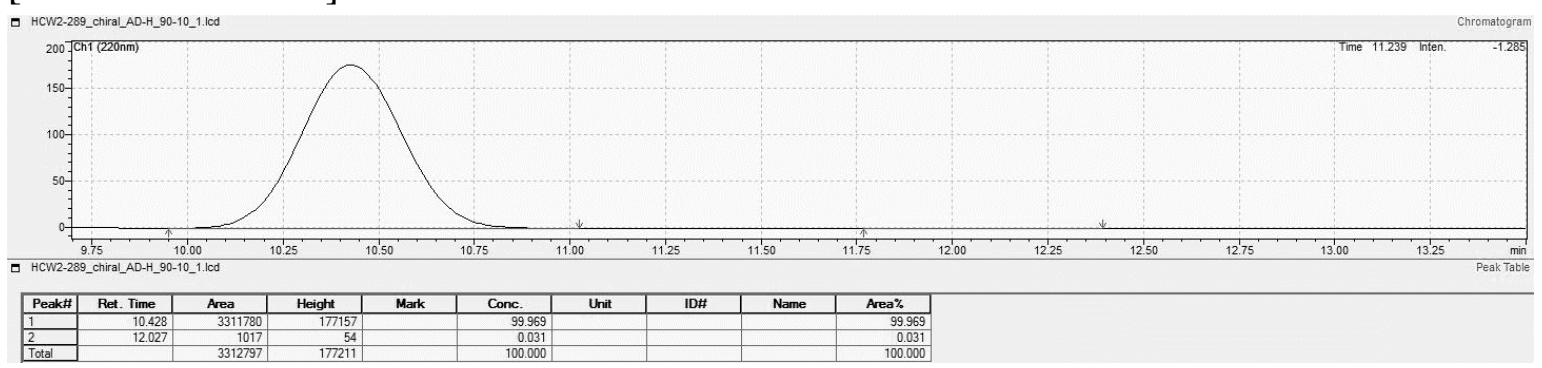

\subsection{Procedure for Mitsunobu cyclization}

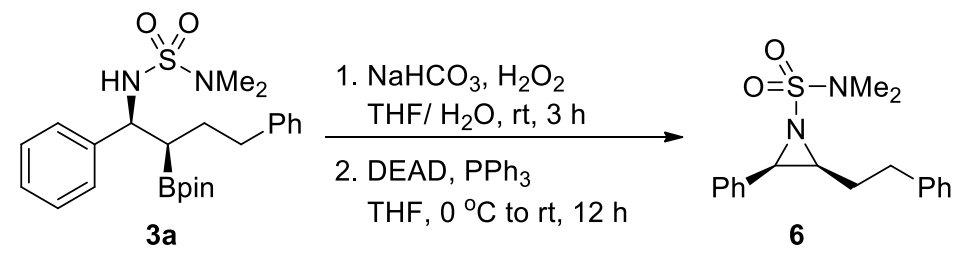

(2S,3R)- $N, N$-Dimethyl-2-phenethyl-3-phenylaziridine-1-sulfonamide (Scheme 3, 6): Mitsunobu cyclization was conducted according to a literature procedure. ${ }^{9}$ To a $25 \mathrm{~mL}$ round bottomed flask equipped with a magnetic stir bar were added the above obtained $\beta$-amino alcohol 5 (972 mg, $2.79 \mathrm{mmol}$, >20:1 d.r., >99\% ee) and $\mathrm{PPh}_{3}(879 \mathrm{mg}, 3.35 \mathrm{mmol}$ ) in THF $(5.6 \mathrm{~mL})$ under argon atmosphere. The reaction mixture was cooled to $0{ }^{\circ} \mathrm{C}$ and diethyl azodicarboxylate (DEAD, $0.525 \mathrm{~mL}, 3.35 \mathrm{mmol}$ ) was added. The mixture was allowed to room temperature and stirred for $12 \mathrm{~h}$. The solvent was removed under reduced pressure and ${ }^{1} \mathrm{H}-\mathrm{NMR}$ analysis of the crude mixture indicated $>20: 1$ diastereoselectivity. The crude mixture was purified by silica gel column chromatography (EtOAc : $n$-hexane $=1: 5$ ) to give the corresponding aziridine 6 as a white liquid $\left(894 \mathrm{mg}, 97 \%,>20: 1\right.$ d.r., $>99 \%$ ee); $[\alpha]_{\mathrm{D}}^{25}$ $68.2\left(c 0.61, \mathrm{CH}_{2} \mathrm{Cl}_{2}\right)$; $\mathrm{NMR}\left(500 \mathrm{MHz}, \mathrm{CDCl}_{3}\right) \delta 7.37-7.29(\mathrm{~m}, 5 \mathrm{H}), 7.24-7.21(\mathrm{t}, J=$ $7.4 \mathrm{~Hz}, 2 \mathrm{H}), 7.17-7.14(\mathrm{~m}, 1 \mathrm{H}), 7.04-7.03(\mathrm{~d}, J=7.3 \mathrm{~Hz}, 2 \mathrm{H}), 3.85-3.84(\mathrm{~d}, J=7.2 \mathrm{~Hz}$, $1 \mathrm{H}), 3.01-2.96(\mathrm{q}, J=6.9 \mathrm{~Hz}, 1 \mathrm{H}), 2.93(\mathrm{~s}, 6 \mathrm{H}), 2.73-2.67(\mathrm{~m}, 1 \mathrm{H}), 2.64-2.59(\mathrm{~m}, 1 \mathrm{H})$, $1.75-1.68(\mathrm{~m}, 1 \mathrm{H}), 1.60-1.53(\mathrm{~m}, 1 \mathrm{H}) ;{ }^{13} \mathrm{C} \mathrm{NMR}\left(126 \mathrm{MHz}, \mathrm{CDCl}_{3}\right) \delta 140.9,133.3,128.6$, 128.5, 128.0, 127.5, 126.2, 46.5, 44.9, 38.7, 33.0, 28.3; HRMS (EI) $\mathrm{m} / \mathrm{z}$ calcd. for $\mathrm{C}_{18} \mathrm{H}_{22} \mathrm{~N}_{2} \mathrm{O}_{2} \mathrm{~S}[\mathrm{M}]^{+}:$330.1402, found: 330.1404; CHIRALCEL OD-H, $n$-hexane $: i$-PrOH $=$ 98:2, $0.5 \mathrm{~mL} / \mathrm{min}, 25^{\circ} \mathrm{C}, \lambda=220 \mathrm{~nm}$; $\mathrm{t}_{\mathrm{R}} 16.8 \mathrm{~min}$ (minor), $\mathrm{t}_{\mathrm{R}} 18.0 \mathrm{~min}$ (major); Analogous mixtures of enantiomers were prepared using racemic $( \pm)-\mathbf{3 a}$ as a substrate under the standard reaction procedure. 
[Racemic 6]

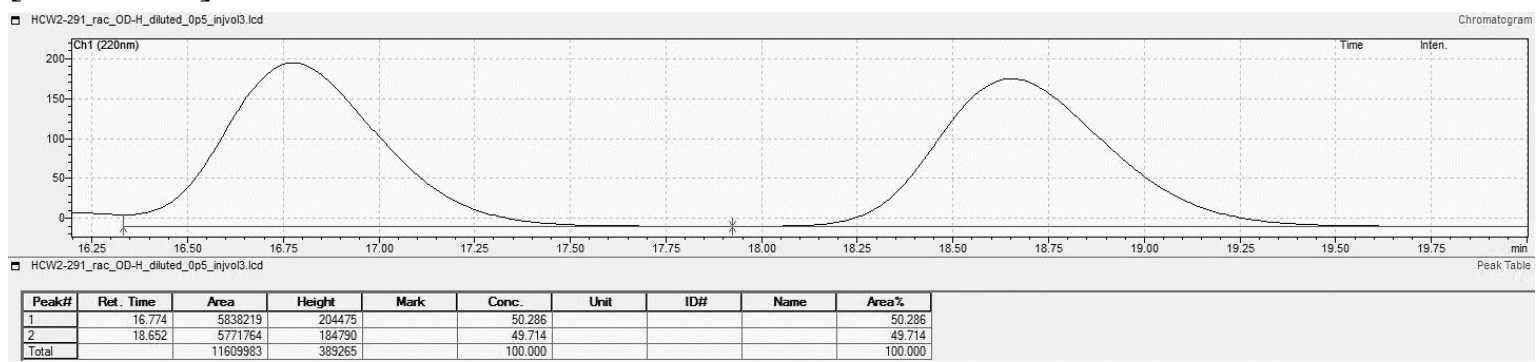

[Enantioenriched 6]

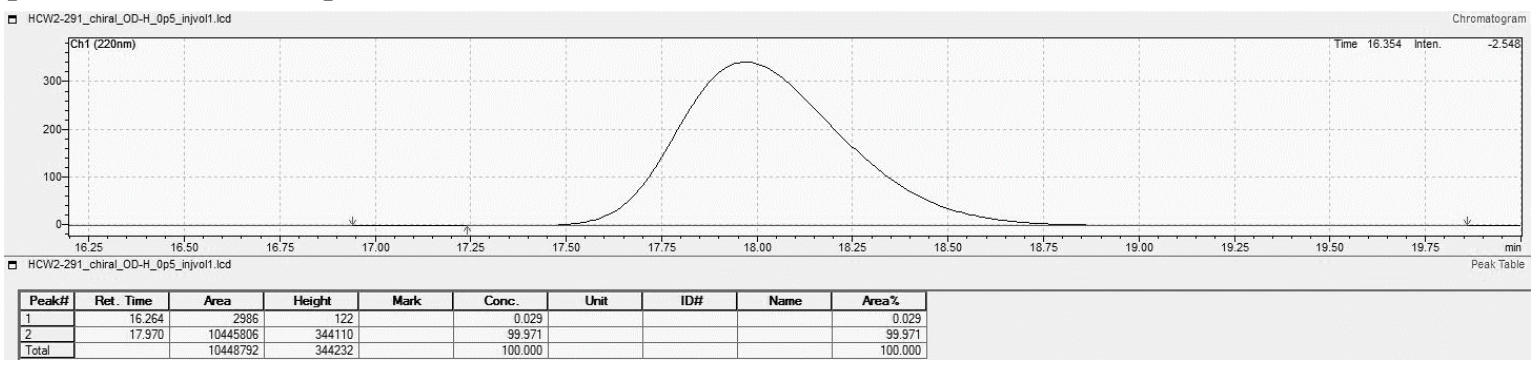

4.5 Procedure for deprotection of $N, N$-dimethylsulfamoyl group

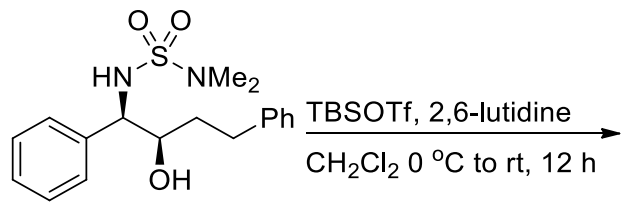

5

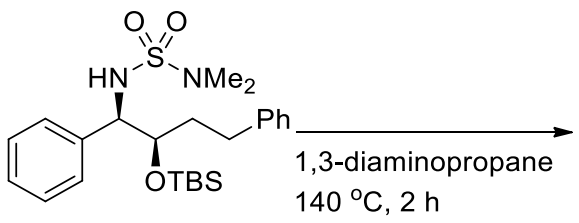

TBS protected 5<smiles>N[C@@H](c1ccccc1)C([O+])CCc1ccccc1</smiles>

7

(1R,2R)-2-((tert-Butyldimethylsilyl)oxy)-1,4-diphenylbutan-1-amine (Scheme 3, 7): The deprotection of $\mathrm{N}, \mathrm{N}$-dimethylsulfamoyl group was conducted according to a literature procedure. ${ }^{7}$ To a $10 \mathrm{~mL}$ round bottomed flask equipped with a magnetic stir bar were added enantiopure $\beta$-aminoalcohol 5 (70 mg, $0.20 \mathrm{mmol},>20: 1$ d.r., >99\% ee) and 2,6-lutidine $(58 \mu \mathrm{L} \mathrm{g}, 0.50 \mathrm{mmol})$ in $\mathrm{CH}_{2} \mathrm{Cl}_{2}(2.0 \mathrm{~mL})$ at $0{ }^{\circ} \mathrm{C}$. To this solution, TBSOTf ( $69 \mu \mathrm{L}, 0.30$ mmol) was slowly added and stirred at room temperature for $12 \mathrm{~h}$. The reaction mixture was poured into a separatory funnel and water $(10 \mathrm{~mL})$ was added. The aqueous phase was extracted with $\mathrm{CH}_{2} \mathrm{Cl}_{2}(10 \mathrm{~mL} \times 3)$ and the combined organic layers were dried over $\mathrm{MgSO}_{4}$, filtered, and concentrated under reduced pressure. ${ }^{1} \mathrm{H}-\mathrm{NMR}$ analysis of the crude mixture indicated >20:1 diastereoselectivity. The crude mixture was purified by silica gel column chromatography (EtOAc : $n$-hexane $=1: 10$ ) to give the corresponding TBS-protected $\mathbf{5}$ as a colorless liquid (93 mg, 99\%).

To a $10 \mathrm{~mL}$ round bottomed flask equipped with a magnetic stir bar were added the above obtained TBS-protected $5(0.20 \mathrm{mmol}, 93 \mathrm{mg})$ and 1,3-diaminopropane $(0.40 \mathrm{~mL})$. The reaction mixture was stirred at $140^{\circ} \mathrm{C}$ for $2 \mathrm{~h}$. After cooled to room temperature, the reaction mixture was quenched with water $(10 \mathrm{~mL})$. The aqueous phase was extracted with $\mathrm{CH}_{2} \mathrm{Cl}_{2}$ $(10 \mathrm{~mL} \times 3)$ and the combined organic layers were dried over $\mathrm{Na}_{2} \mathrm{SO}_{4}$, filtered, and concentrated under reduced pressure to give analytically pure product $\mathbf{7}$ as a colorless liquid (89 mg, 96\%, >20:1 d.r., >99\% ee); $[\alpha]_{\mathrm{D}}^{23}-25.8\left(c 0.69, \mathrm{CH}_{2} \mathrm{Cl}_{2}\right) ;{ }^{1} \mathrm{H}$ NMR (500 MHz, $\left.\mathrm{CDCl}_{3}\right) \delta 7.35-7.34(\mathrm{~d}, J=4.2 \mathrm{~Hz}, 4 \mathrm{H}), 7.29-7.26(\mathrm{~m}, 3 \mathrm{H}), 7.20-7.17(\mathrm{t}, J=7.4 \mathrm{~Hz}$, $1 \mathrm{H}), 7.14-7.12(\mathrm{~d}, J=7.2 \mathrm{~Hz}, 2 \mathrm{H}), 4.03-4.02(\mathrm{~d}, J=5.6 \mathrm{~Hz}, 1 \mathrm{H}), 3.83-3.80(\mathrm{dd}, J=$ $10.3,5.9 \mathrm{~Hz}, 1 \mathrm{H}), 2.69-2.66(\mathrm{t}, J=8.3 \mathrm{~Hz}, 2 \mathrm{H}), 1.97-1.88(\mathrm{~m}, 3 \mathrm{H}), 1.69-1.62(\mathrm{~m}, 1 \mathrm{H})$, 
0.94 (s, 9H), 0.03 (s, 3H), -0.13 (s, 3H); $\left.{ }^{13} \mathrm{C} \mathrm{NMR} \mathrm{(126} \mathrm{MHz,} \mathrm{CDCl}_{3}\right) \delta 143.7,142.3,128.5$, 128.4, 128.4, 127.4, 127.3, 125.9, 59.1, 36.2, 31.3, 26.1, 18.3, -4.2, -4.8; HRMS (FAB) m/z calcd. for $\mathrm{C}_{22} \mathrm{H}_{34} \mathrm{NOSi}[\mathrm{M}+\mathrm{H}]^{+}:$356.2410, found: 356.2412; CHIRALPAK AD-H, nhexane : $i-\mathrm{PrOH}=95: 5,1 \mathrm{~mL} / \mathrm{min}, 40^{\circ} \mathrm{C}, \lambda=220 \mathrm{~nm}$; $\mathrm{t}_{\mathrm{R}} 2.7$ min (major), $\mathrm{t}_{\mathrm{R}} 2.9 \min$ (minor); Analogous mixtures of enantiomers were prepared using racemic $( \pm)-5$ as a substrate under the standard reaction procedure.

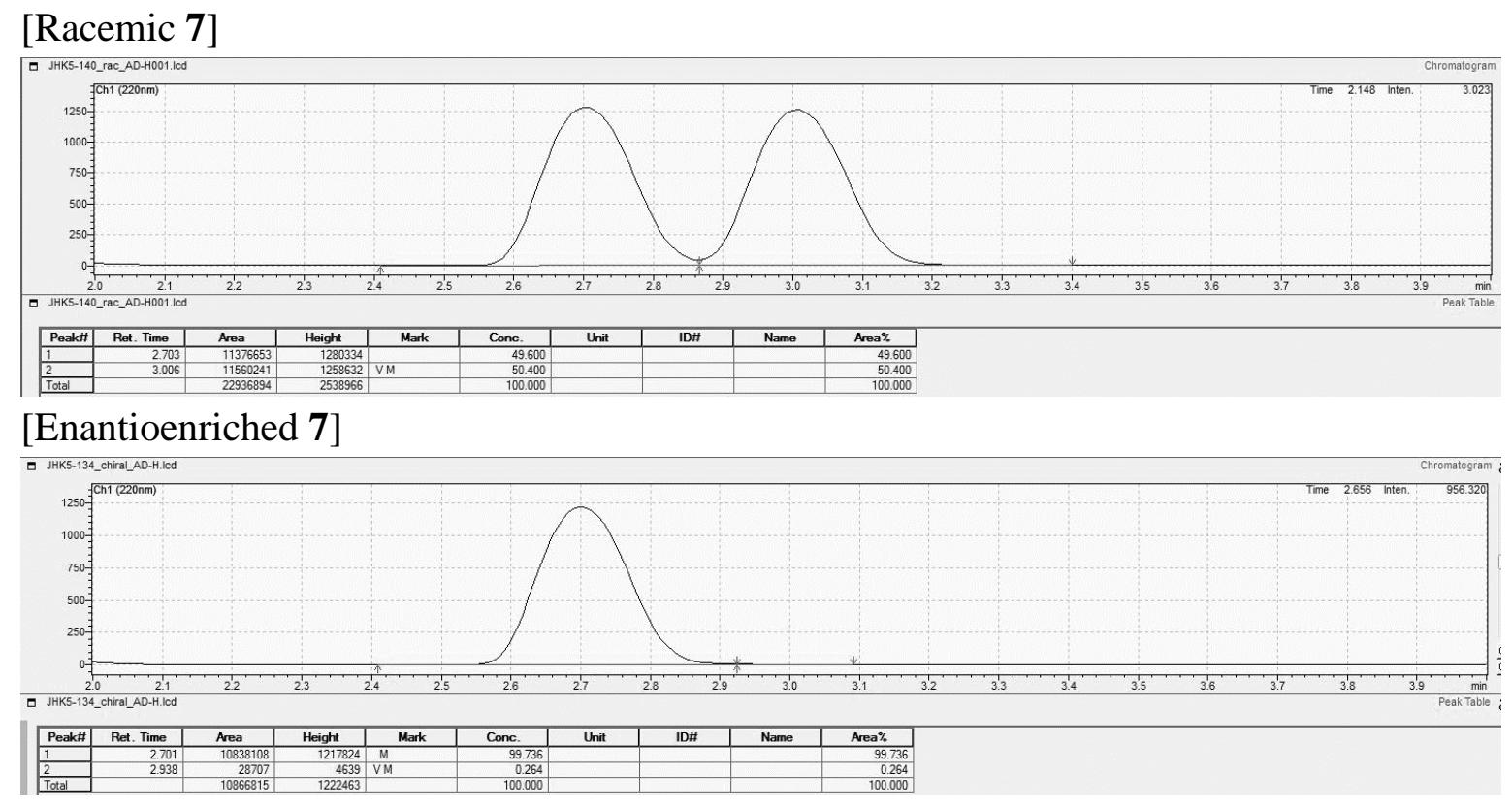

\section{References}

1. Bernsmann, H.; van den Berg, M.; Hoen, R.; Minnaard, A. J.; Mehler, G.; Reetz, M. T.; De Vries, J. G.; Feringa, B. L., PipPhos and MorfPhos: Privileged Monodentate Phosphoramidite Ligands for Rhodium-Catalyzed Asymmetric Hydrogenation. J. Org. Chem. 2005, 70, 943-951.

2. Peña, D.; Minnaard, A. J.; de Vries, J. G.; Feringa, B. L., Highly Enantioselective Rhodium-Catalyzed Hydrogenation of $\beta$-Dehydroamino Acid Derivatives Using Monodentate Phosphoramidites. J. Am. Chem. Soc. 2002, 124, 14552-14553.

3. Hong, K.; Liu, X.; Morken, J. P., Simple Access to Elusive $\alpha$-Boryl Carbanions and Their Alkylation: An Umpolung Construction for Organic Synthesis. J. Am. Chem. Soc. 2014, 136, 10581-10584.

4. Lee,Y.; Baek, S.-y.; Park, J.; Kim, S.-T.; Tussupbayev, S.; Kim, J.; Baik, M.-H.; Cho, S. H., Chemoselective Coupling of 1,1-Bis[(pinacolato)boryl]alkanes for the Transition-MetalFree Borylation of Aryl and Vinyl Halides: A Combined Experimental and Theoretical Invesitigation. J. Am. Chem. Soc. 2017, 139, 976-984.

5. Joannou, M. V.; Moyer, B. S.; Goldfogel, M. J.; Meek, S. J., Silver(I)-Catalyzed Diastereoselective Synthesis of anti-1,2-Hydroxyboronates. Angew. Chem., Int. Ed. 2015, $54,14141-14145$.

6. Jo, W.; Kim, J.; Choi, S.; Cho, S. H., Transition-Metal-Free Regioselective Alkylation of Pyridine $\mathrm{N}$-Oxides Using 1,1-Diborylalkanes as Alkylating Reagents. Angew. Chem., Int. Ed. 2016, 55, 9690-9694. 
7. Li, J.; Minnaard, A. J.; Gebbink, R. J. M. K.; van Koten, G., PCP-Pincer Palladium Complex-Catalyzed Allylation of $N, N$-Dimethylsulfamoyl-Protected Aldimines. Tetrahedron Lett. 2009, 50, 2232-2235.

8. Sonawane, R. P.; Jheengut, V.; Rabalakos, C.; Larouche-Gauthier, R.; Scott, H. K.; Aggarwal, V. K., Enantioselective Construction of Quaternary Stereogenic Centers from Tertiary Boronic Esters: Methodology and Applications. Angew. Chem., Int. Ed. 2011, 50, 3760-3763.

9. Righi, P.; Scardovi, N.; Marotta, E.; ten Holte, P.; Zwanenburg, B., Solution- and SolidPhase Synthesis of 4-Hydroxy-4,5-dihydroisoxazole Derivatives from Enantiomerically Pure N-Tosyl-2,3-aziridine Alcohols. Org. Lett. 2002, 4, 497-500. 
Appendix I

Spectral Copies of ${ }^{1} \mathrm{H},{ }^{11} \mathrm{~B},{ }^{13} \mathrm{C}$ and ${ }^{19} \mathrm{~F}$ NMR Data Obtained in this Study 
$N, N$-Dimethyl- $N^{\prime}$-[(1S,2R)-1,4-diphenyl-2-(4,4,5,5-tetramethyl-1,3,2-dioxaborolan-2yl)butyl]sulfamide (Table 2, 3a)
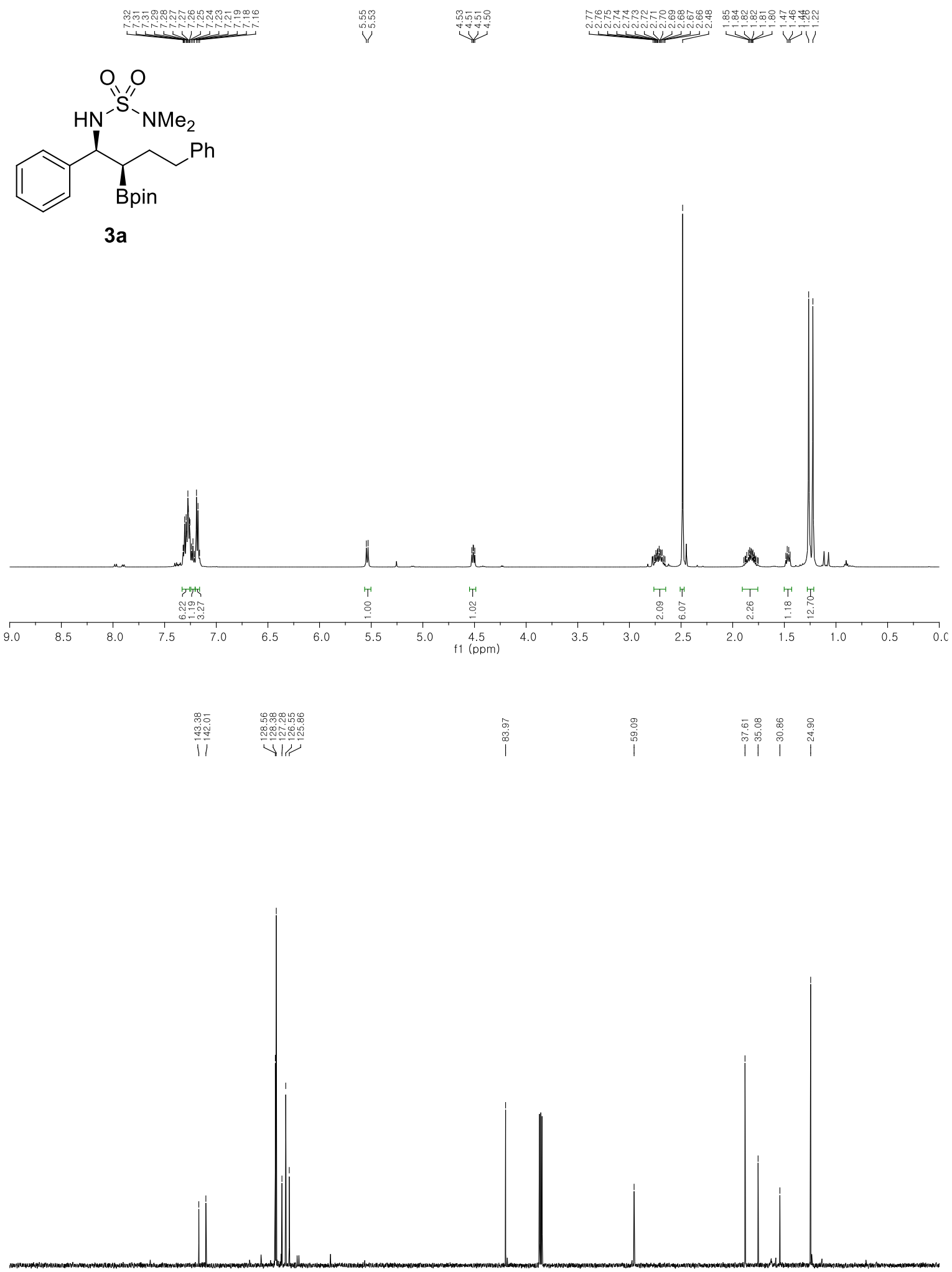

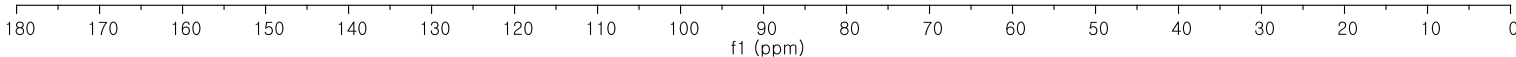


${ }^{11} \mathrm{~B}$ NMR (161 MHz, $\left.\mathrm{CDCl}_{3}\right)$

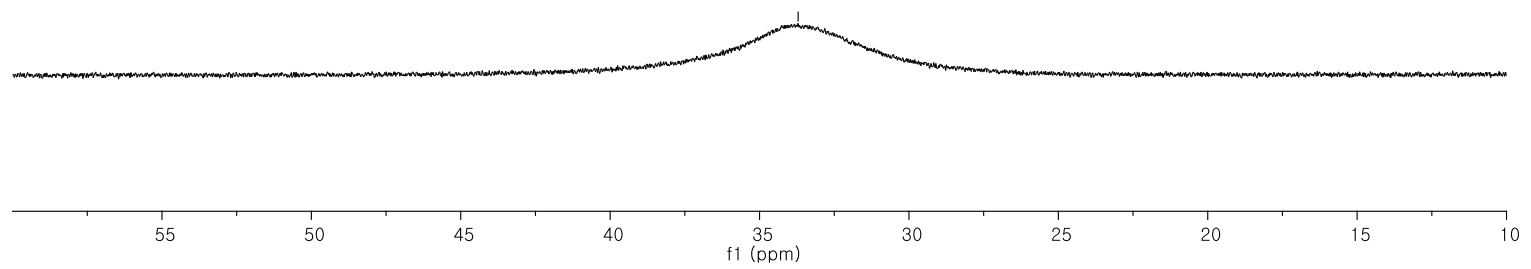


$N, N$-Dimethyl- $N^{\prime}$-[(1S,2R)-4-phenyl-2-(4,4,5,5-tetramethyl-1,3,2-dioxaborolan-2-yl)-1(4-(trifluoromethyl)phenyl)butyl]sulfamide (Table 2, 3b)
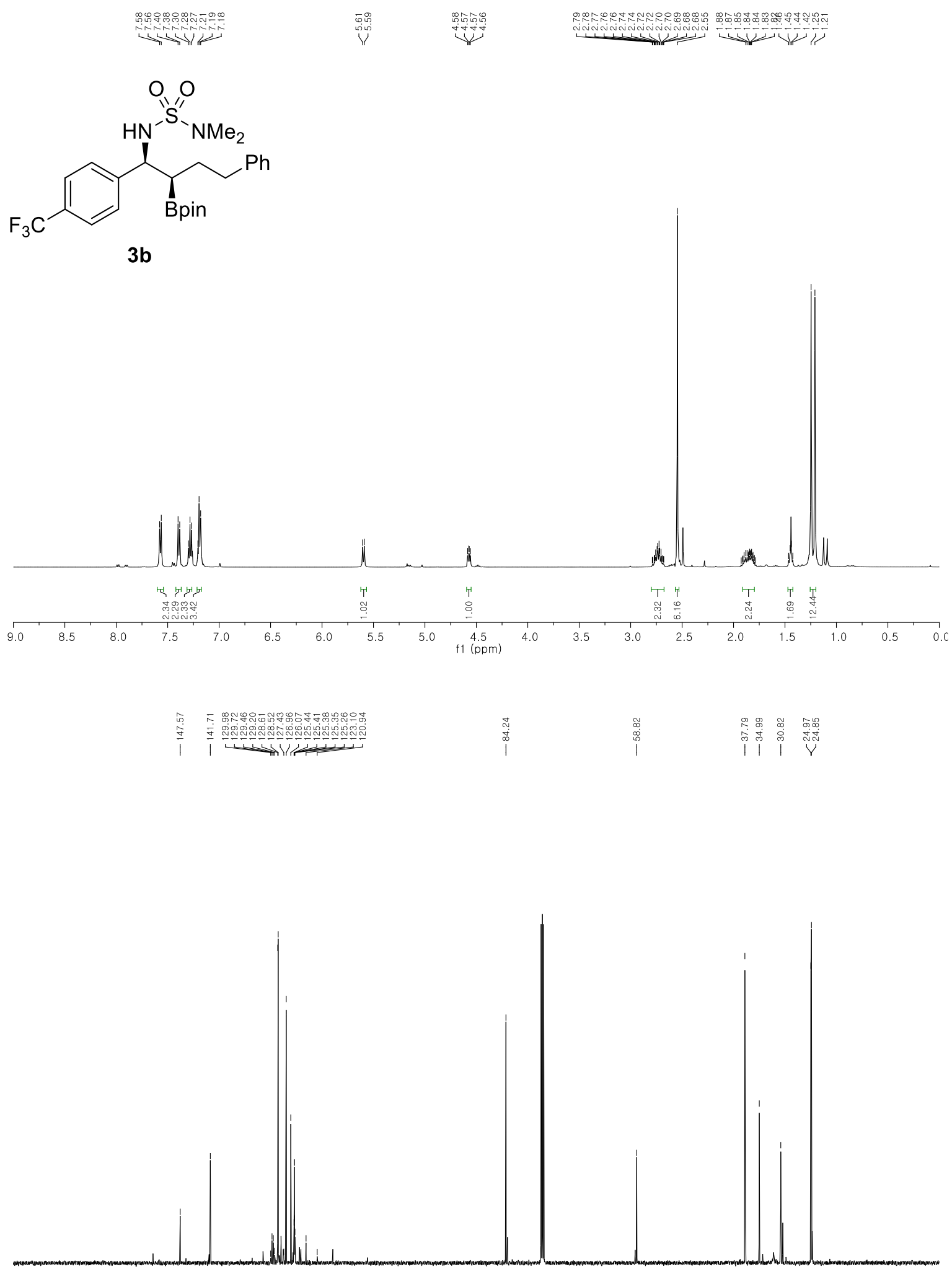

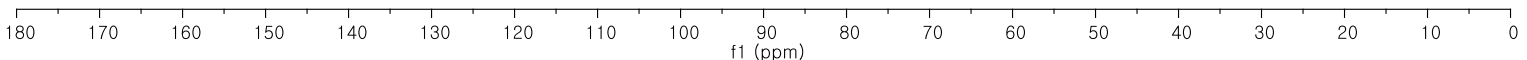


${ }^{19} \mathrm{~F}$ NMR (471 MHz, $\left.\mathrm{CDCl}_{3}\right)$

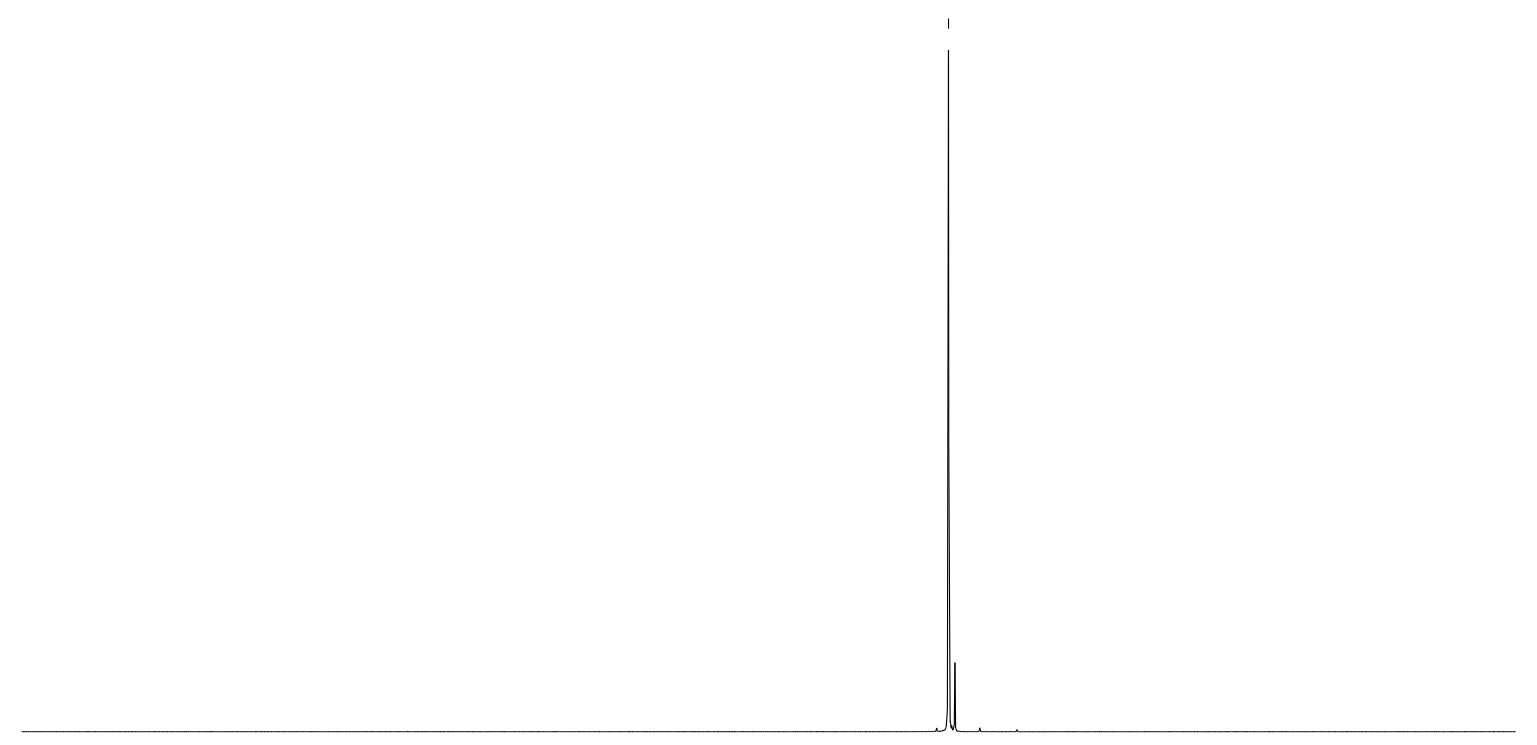

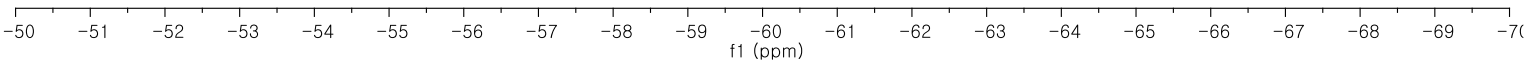

${ }^{11} \mathrm{~B}$ NMR $\left(161 \mathrm{MHz}, \mathrm{CDCl}_{3}\right)$

$55 \quad 50 \quad 45$

40

35
$\mathrm{f} 1(\mathrm{ppm})$

30

25

20

15 

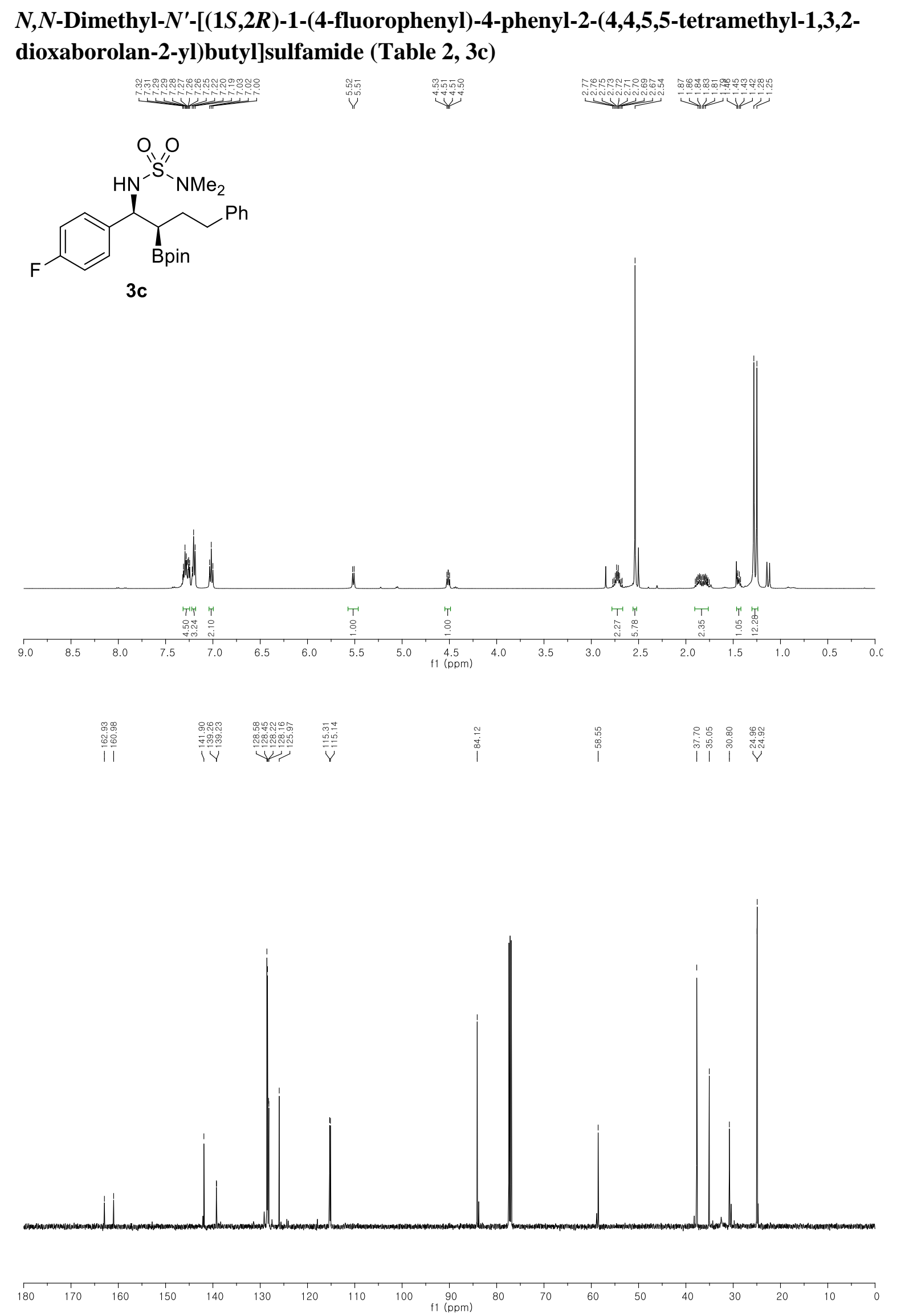

S34 
${ }^{19} \mathrm{~F}$ NMR $\left(471 \mathrm{MHz}, \mathrm{CDCl}_{3}\right)$

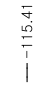
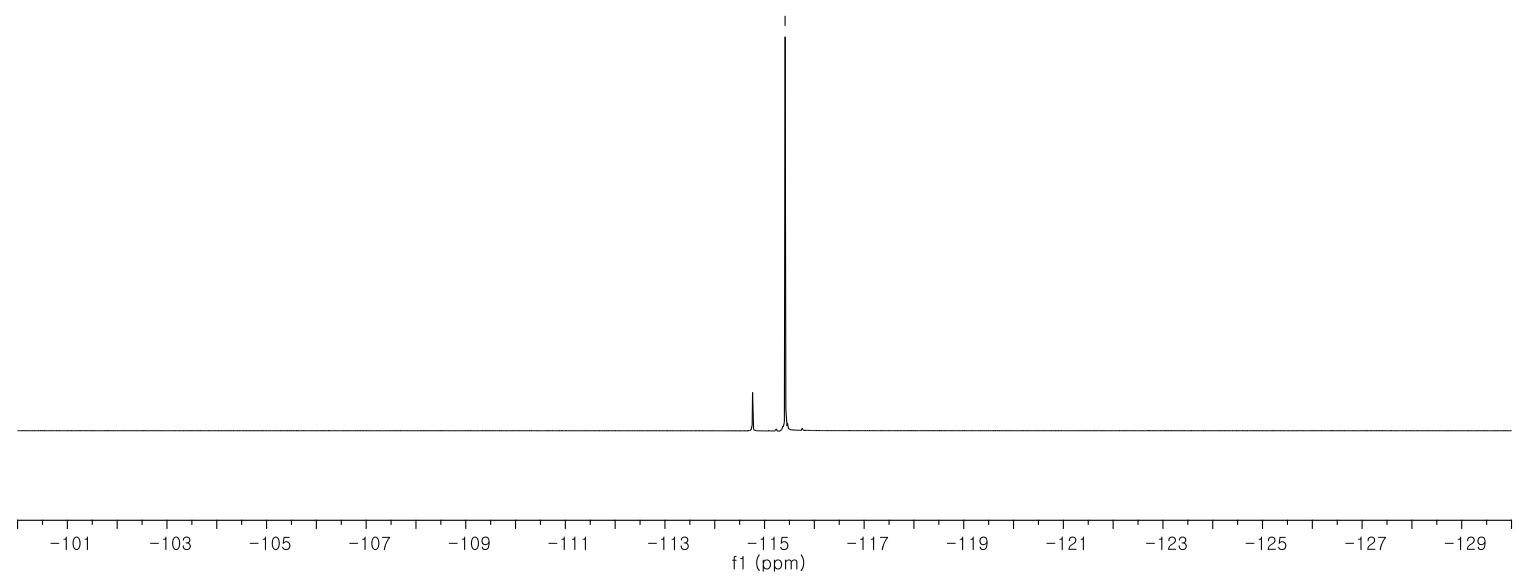

${ }^{11} \mathrm{~B}$ NMR $\left(161 \mathrm{MHz}, \mathrm{CDCl}_{3}\right)$

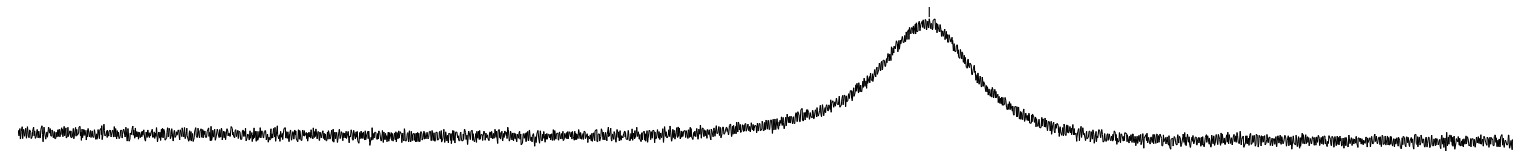

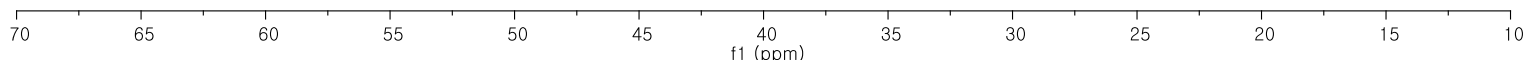


$N, N$-Dimethyl- $N^{\prime}-[(1 S, 2 R)$-1-(4-chlorophenyl)-4-phenyl-2-(4,4,5,5-tetramethyl-1,3,2dioxaborolan-2-yl)butyl]sulfamide (Table 2, 3d)
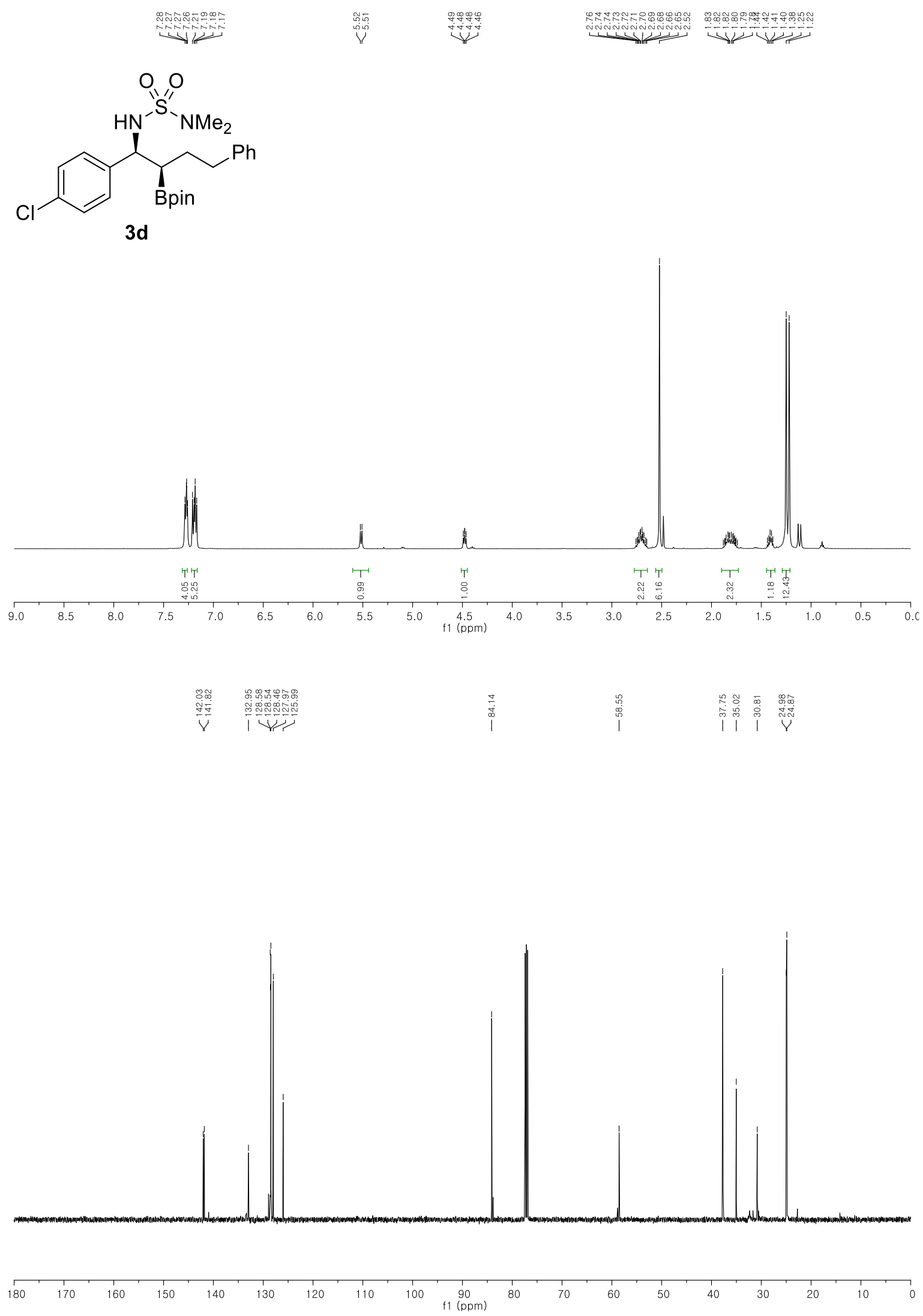

S36 
${ }^{11} \mathrm{~B}$ NMR (161 MHz, $\left.\mathrm{CDCl}_{3}\right)$

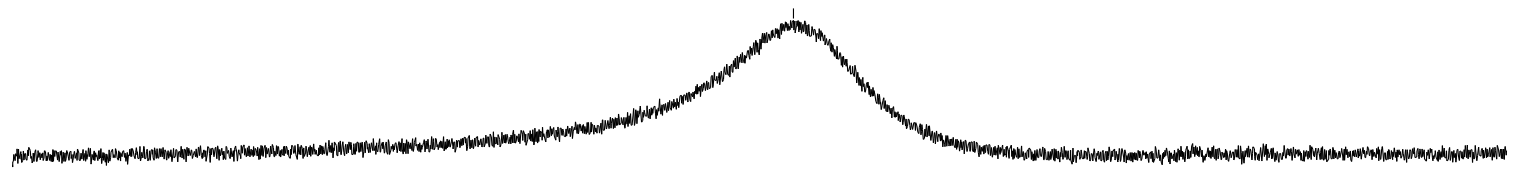

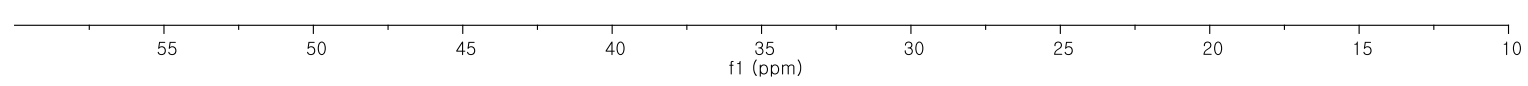


$N, N$-Dimethyl- $N^{\prime}$-[(1S,2R)-1-(4-bromophenyl)-4-phenyl-2-(4,4,5,5-tetramethyl-1,3,2dioxaborolan-2-yl)butyl]sulfamide (Table 2, 3e)
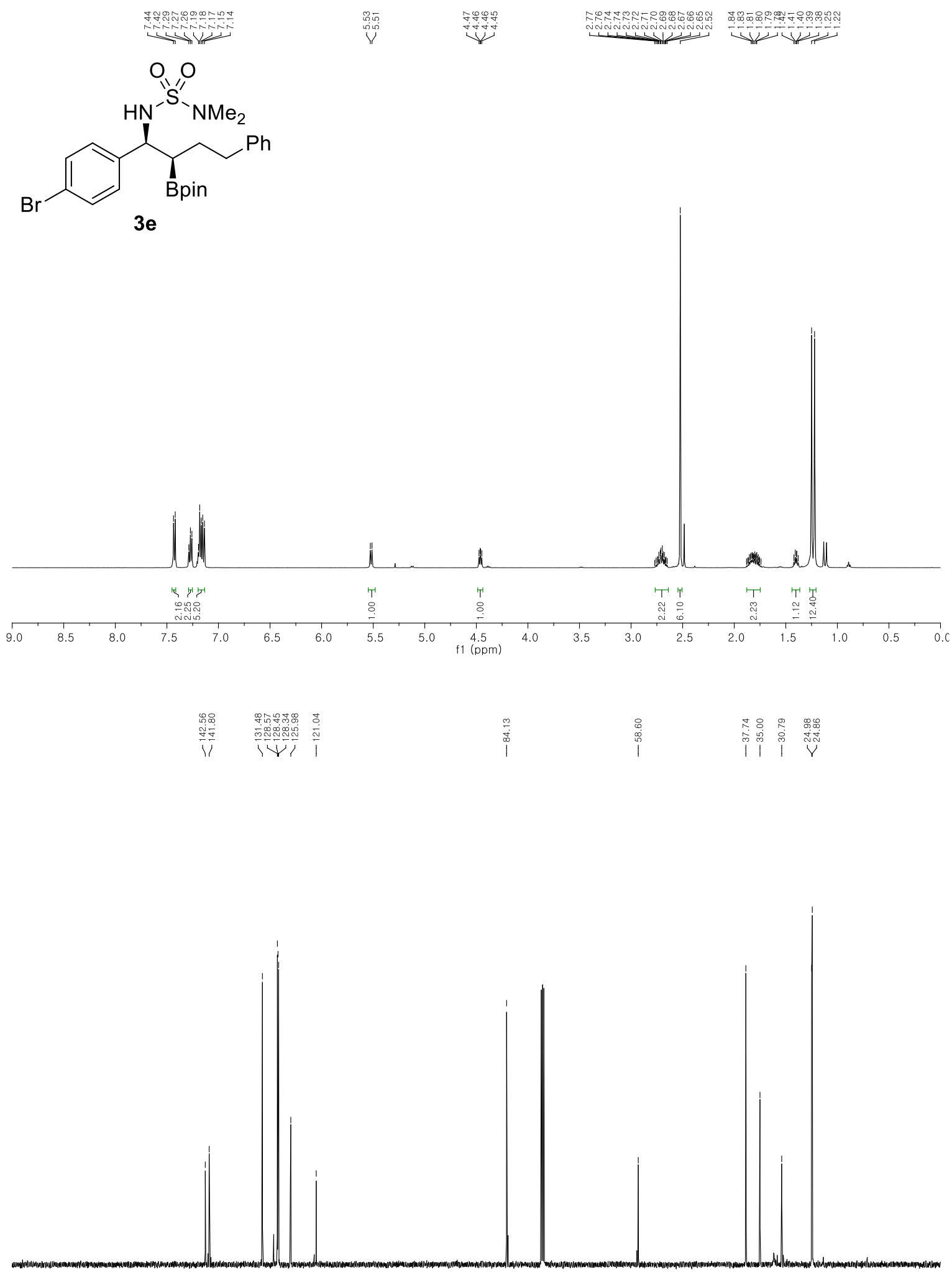

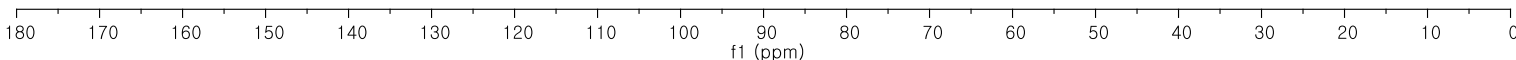


${ }^{11} \mathrm{~B}$ NMR (161 MHz, $\left.\mathrm{CDCl}_{3}\right)$

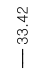

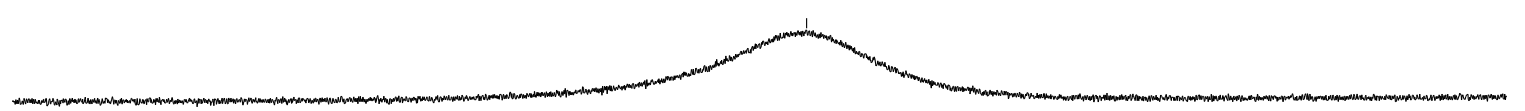

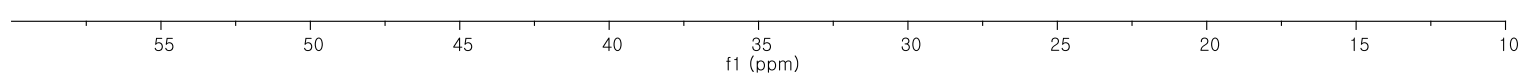


$N, N$-Dimethyl- $N^{\prime}-[(1 S, 2 R)-4-p h e n y l-2-(4,4,5,5-$ tetramethyl-1,3,2-dioxaborolan-2-yl)-1(p-tolyl)butyl]sulfamide (Table 2, 3f)
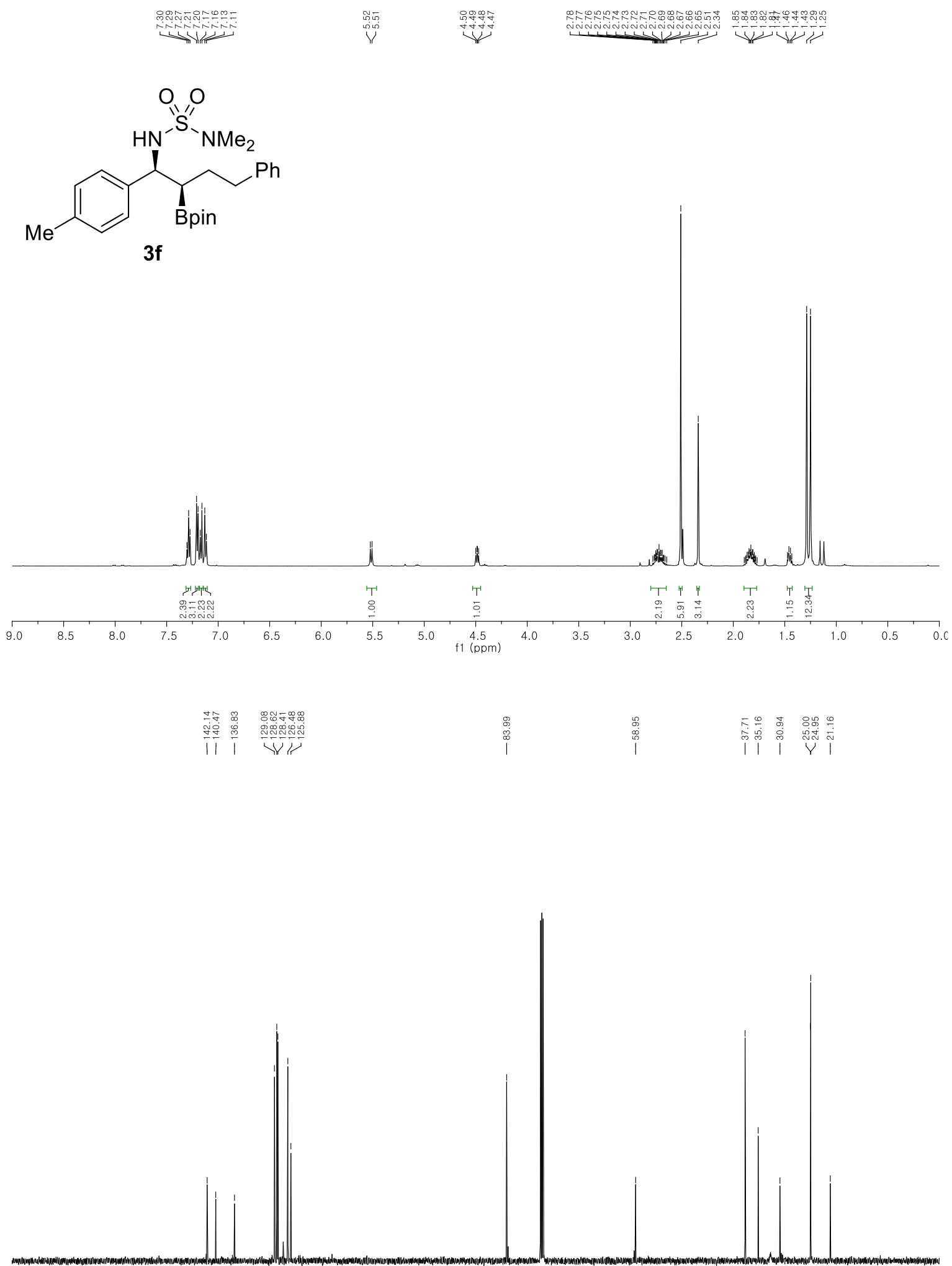

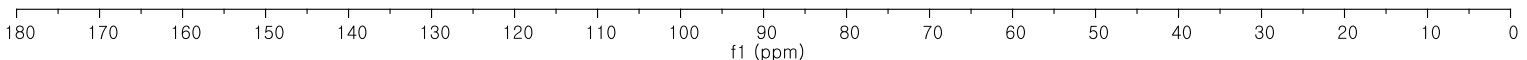


${ }^{11} \mathrm{~B}$ NMR (161 MHz, $\left.\mathrm{CDCl}_{3}\right)$

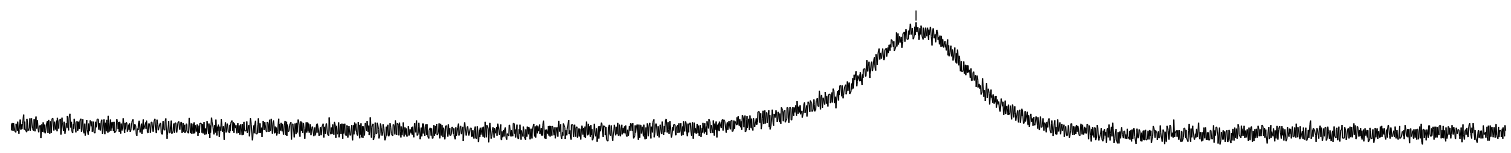

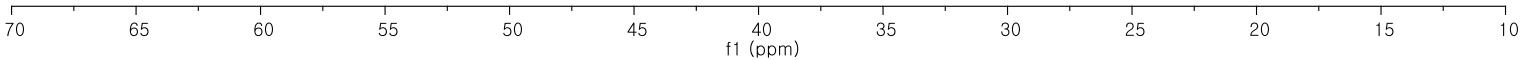


$N, N$-Dimethyl- $N$ '-[(1S,2R)-1-(4-methoxyphenyl)-4-phenyl-2-(4,4,5,5-tetramethyl1,3,2-dioxaborolan-2-yl)butyl]sulfamide (Table 2, 3g)
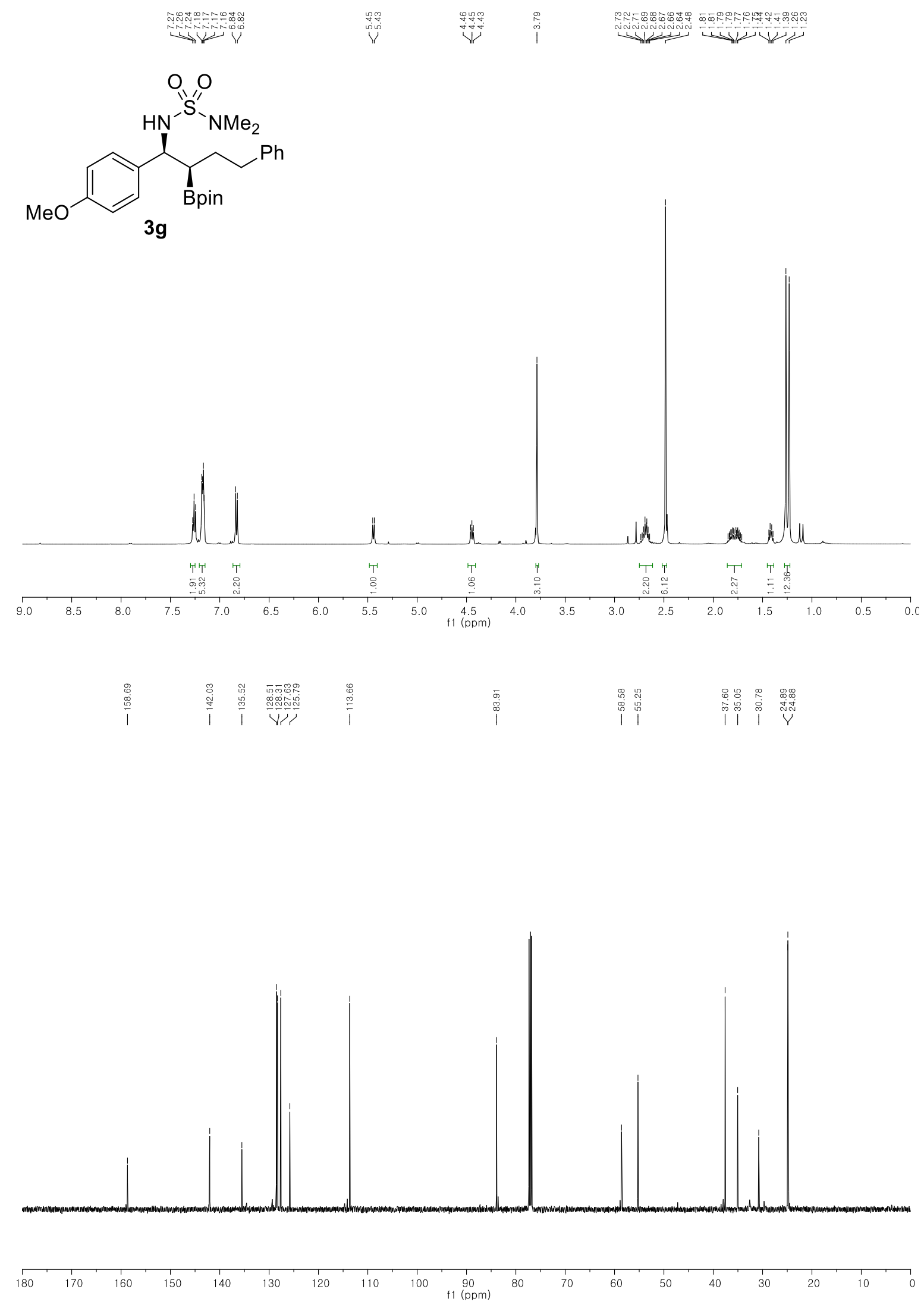

S42 
${ }^{11} \mathrm{~B}$ NMR (161 MHz, $\left.\mathrm{CDCl}_{3}\right)$

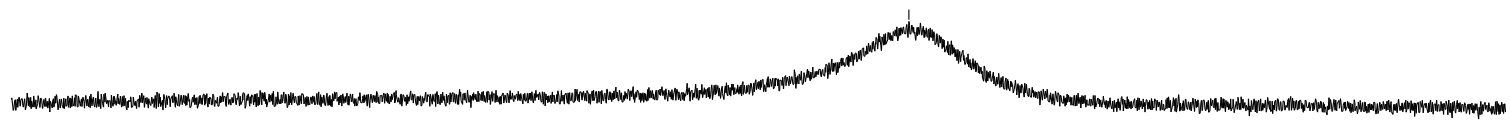

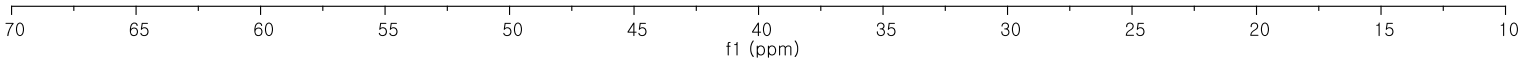


$N, N$-Dimethyl- $N^{\prime}$-[(1S,2R)-1-(3-bromophenyl)-4-phenyl-2-(4,4,5,5-tetramethyl-1,3,2dioxaborolan-2-yl)butyl]sulfamide (Table 2, 3h)

年<smiles>CS(=O)(=O)NC(c1cccc(Br)c1)C(CCc1ccccc1)c1ccccc1</smiles>

$3 \mathrm{~h}$
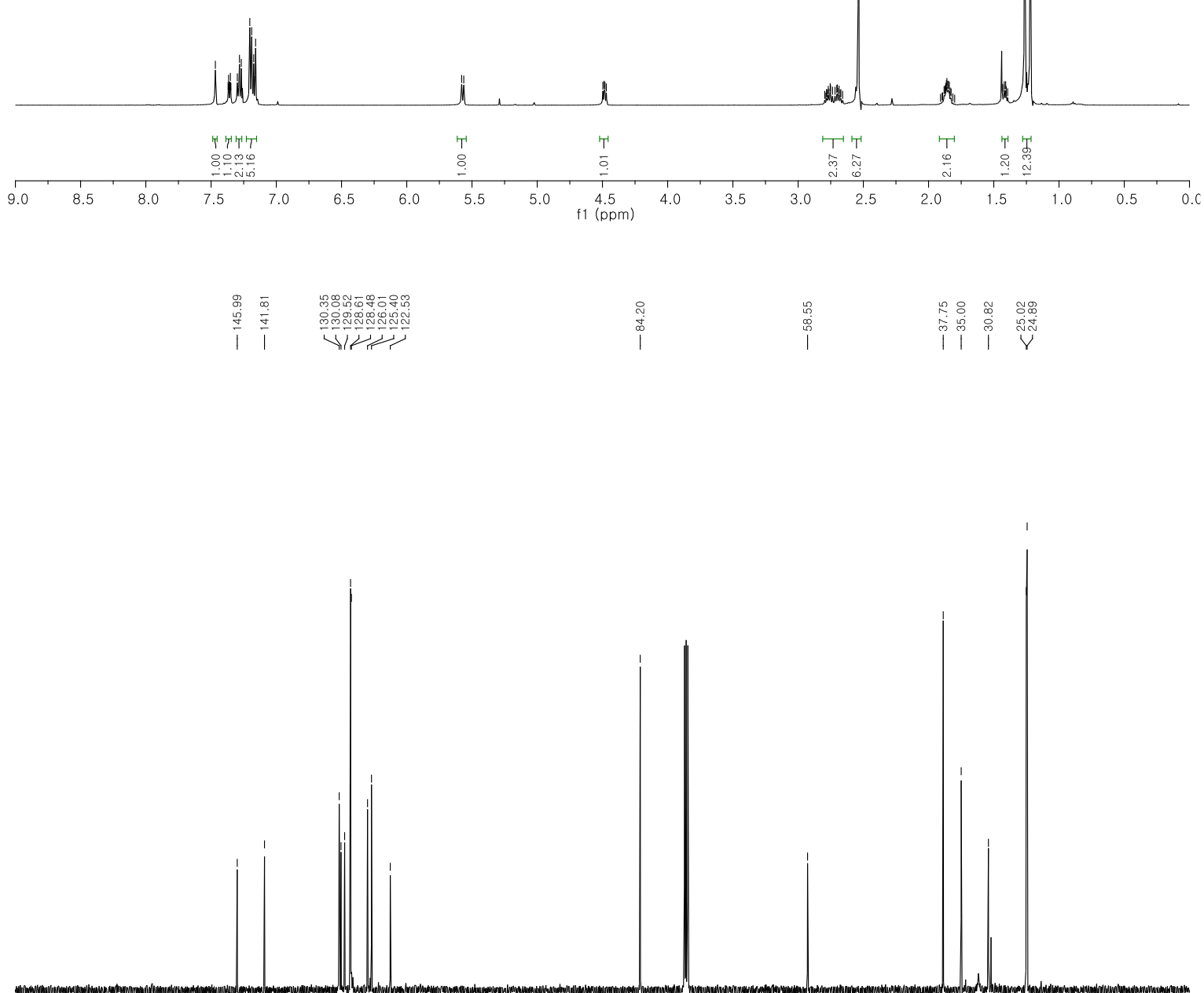

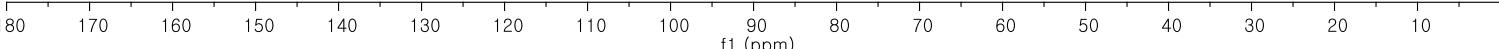


${ }^{11} \mathrm{~B}$ NMR (161 MHz, $\left.\mathrm{CDCl}_{3}\right)$

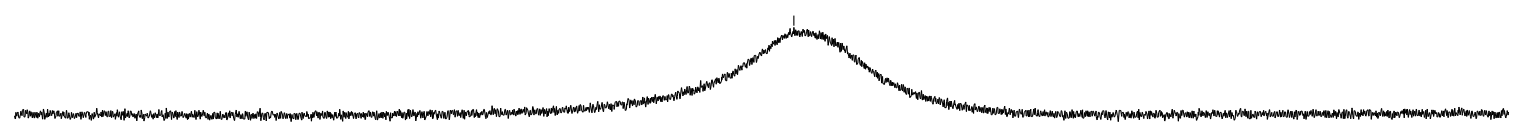

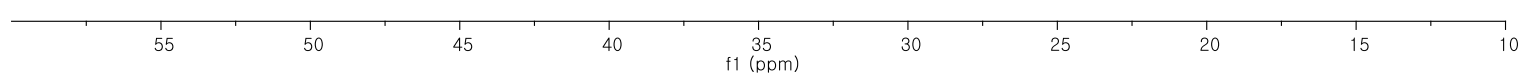


$N, N$-Dimethyl- $N^{\prime}$-[(1S,2R)-4-phenyl-2-(4,4,5,5-tetramethyl-1,3,2-dioxaborolan-2-yl)-1(m-tolyl)butyl]sulfamide (Table 2, 3i)
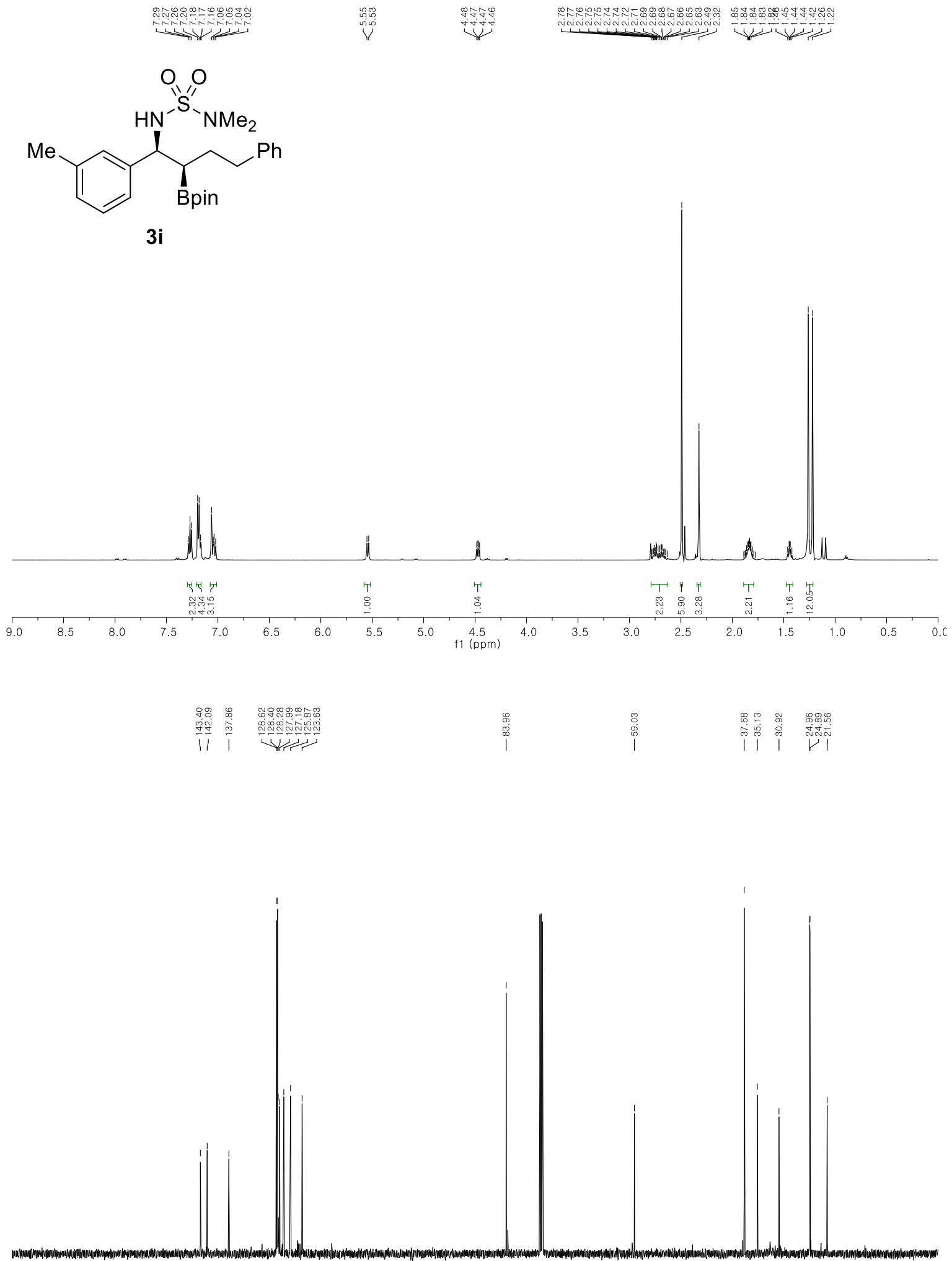

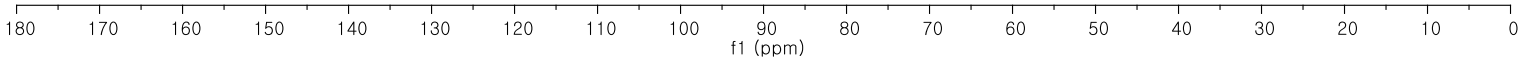


${ }^{11} \mathrm{~B}$ NMR (161 MHz, $\left.\mathrm{CDCl}_{3}\right)$

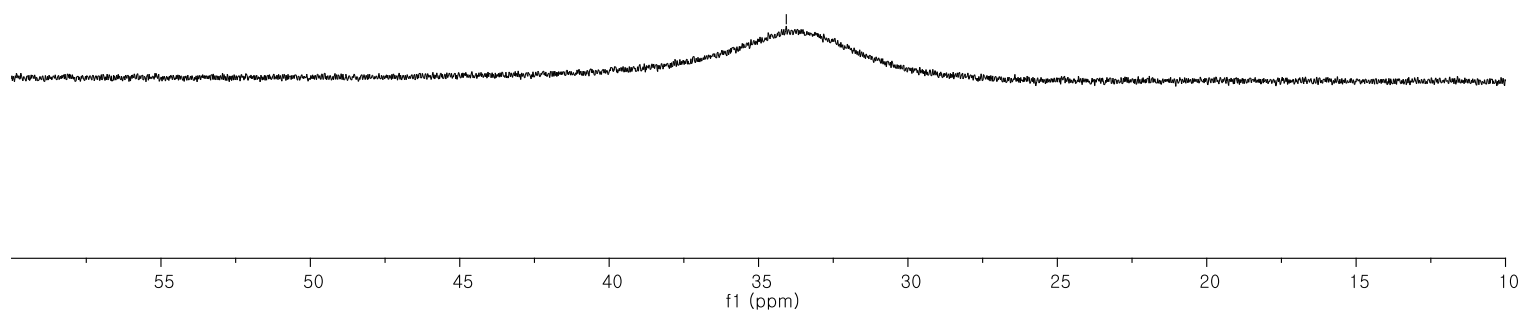


$N, N$-Dimethyl- $N^{\prime}$-[(1S,2R)-4-phenyl-2-(4,4,5,5-tetramethyl-1,3,2-dioxaborolan-2-yl)-1(o-tolyl)butyl]sulfamide (Table 2, 3j)

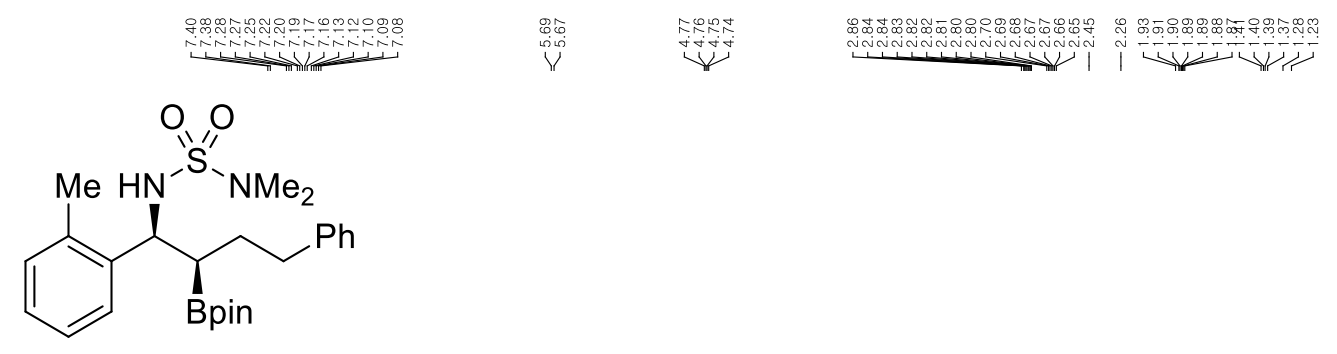

3j
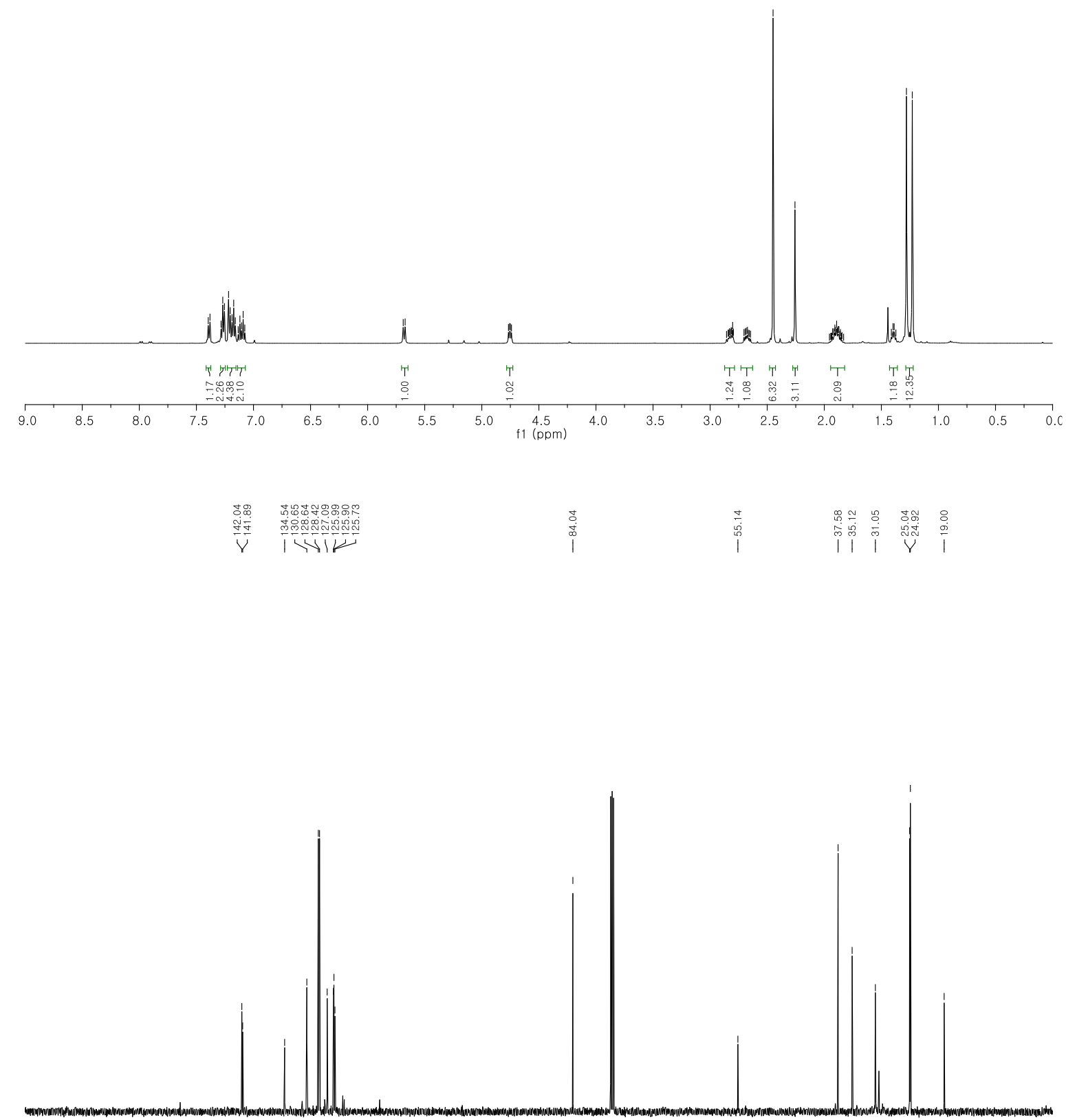

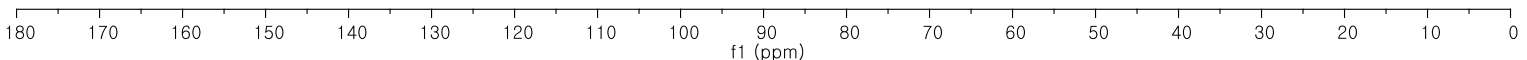


${ }^{11} \mathrm{~B}$ NMR (161 MHz, $\left.\mathrm{CDCl}_{3}\right)$

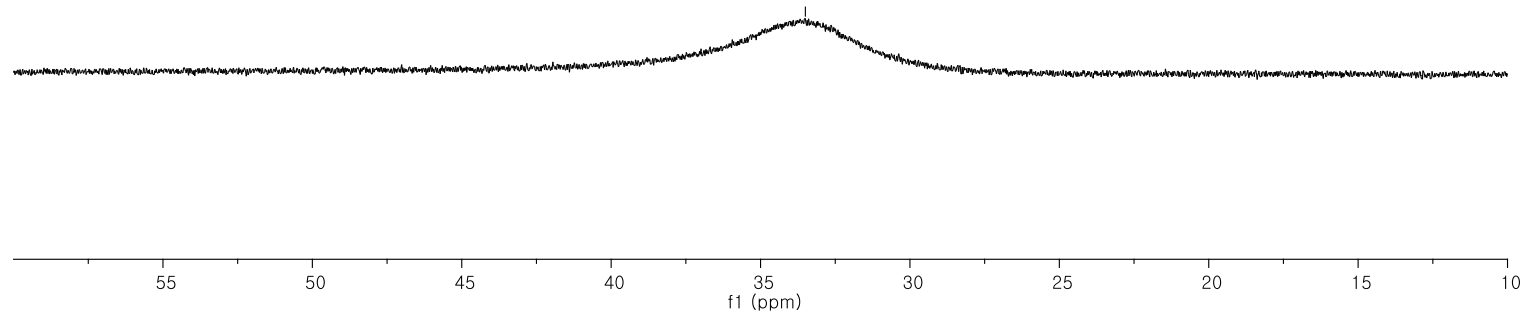


$N, N$-Dimethyl- $N^{\prime}-[(1 S, 2 R)-1-($ naphthalen-2-yl)-4-phenyl-2-(4,4,5,5-tetramethyl-1,3,2dioxaborolan-2-yl)butyl]sulfamide (Table 2, 3k)

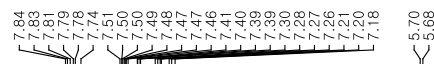<smiles>CNS(=O)(=O)NC(c1ccc2ccccc2c1)C(CCc1ccccc1)c1ccccc1</smiles>

3k
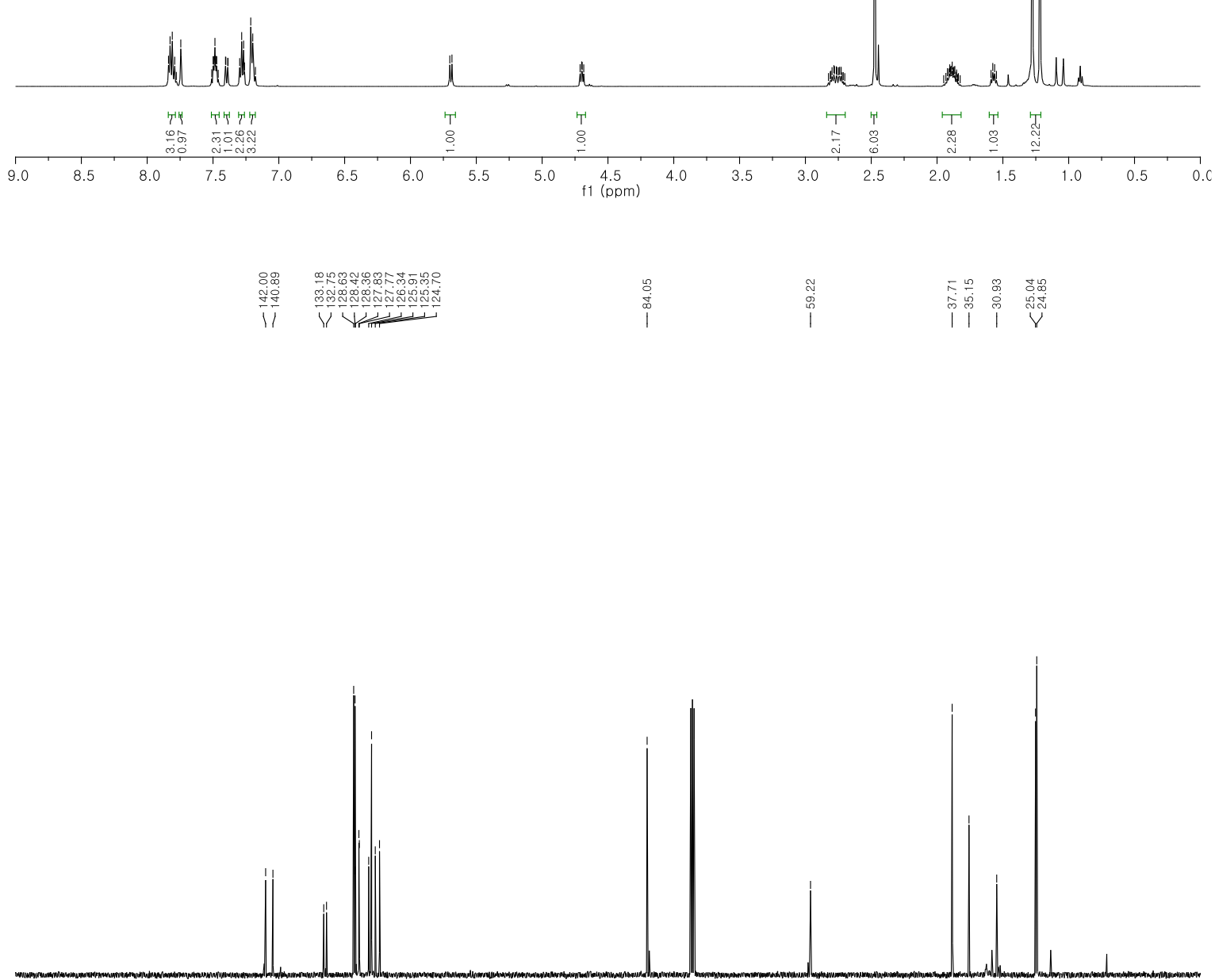

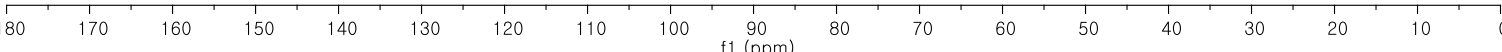


${ }^{11} \mathrm{~B}$ NMR (161 MHz, $\left.\mathrm{CDCl}_{3}\right)$

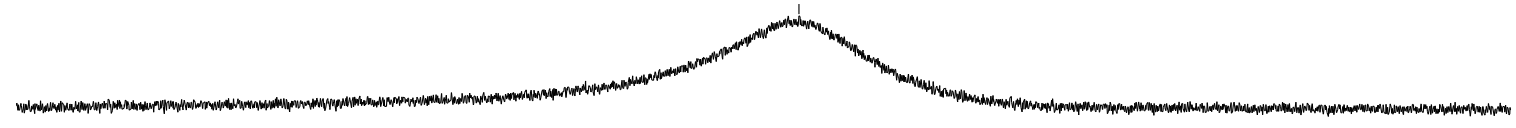

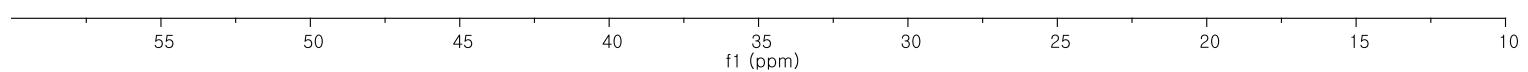


$N, N$-Dimethyl- $N^{\prime}$-[(1S,2R)-4-phenyl-2-(4,4,5,5-tetramethyl-1,3,2-dioxaborolan-2-yl)-1(thiophen-2-yl)butyl]sulfamide (Table 2, 3l)

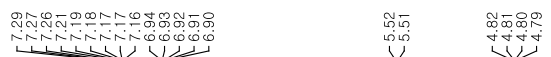

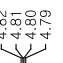

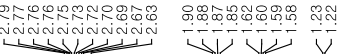<smiles>CNS(=O)(=O)NC(c1cccs1)C(CCc1ccccc1)c1ccccc1</smiles>

31

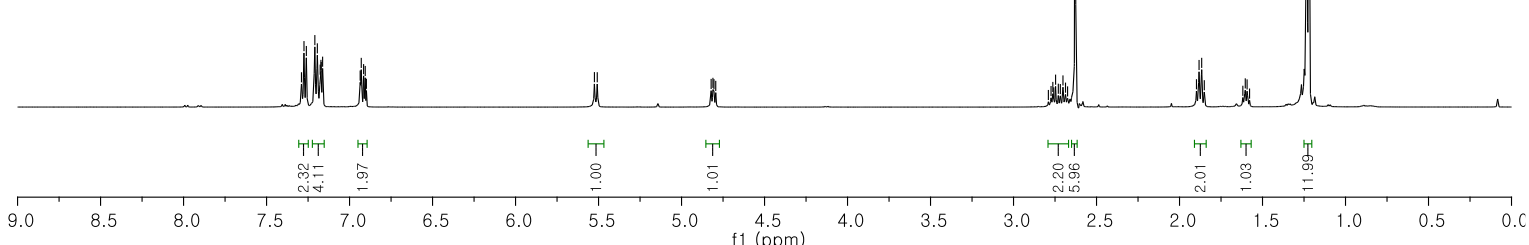

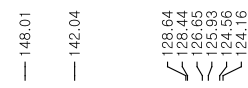

$$
\begin{aligned}
& \infty \\
& \substack{0 \\
+\\
\infty \\
1} \\
& 1
\end{aligned}
$$

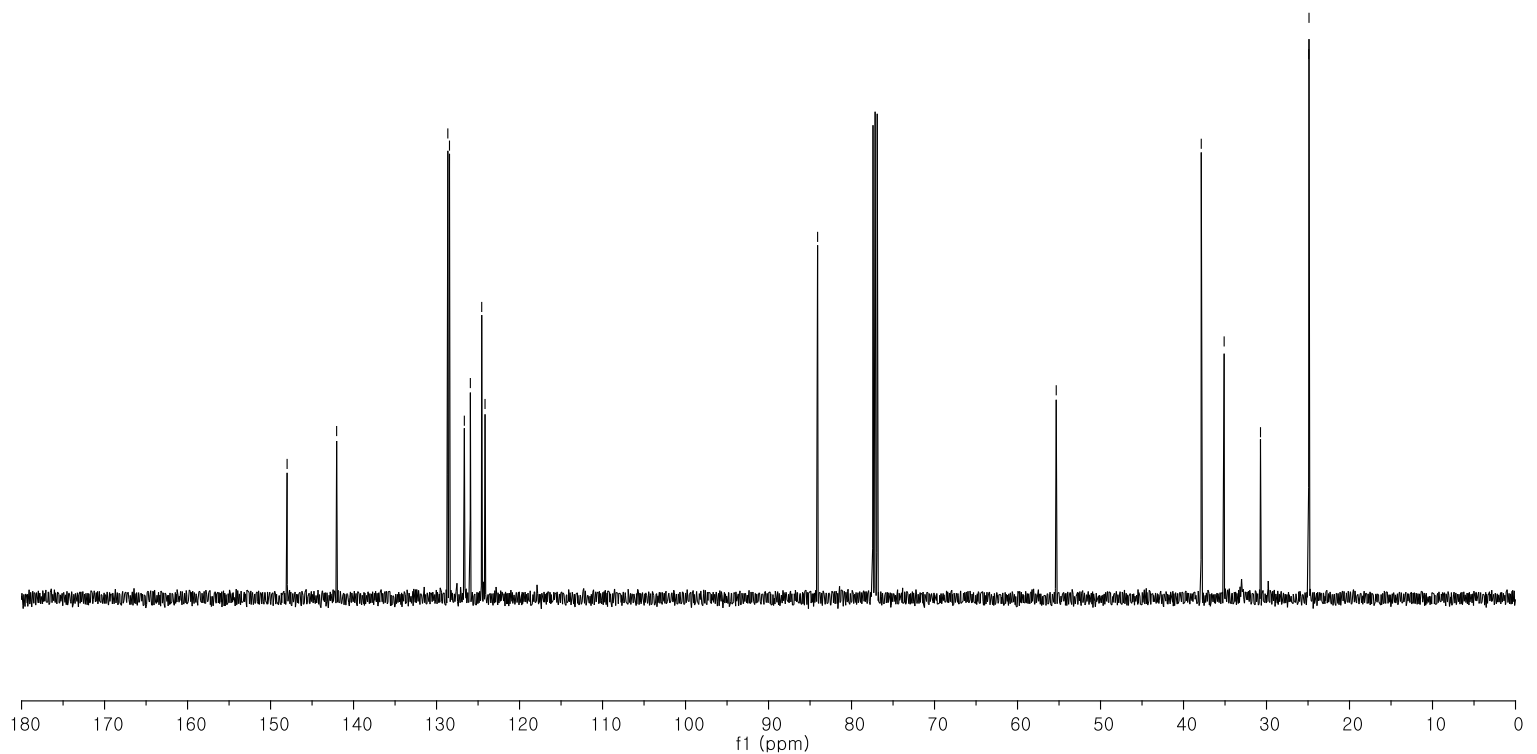


${ }^{11} \mathrm{~B}$ NMR (161 MHz, $\left.\mathrm{CDCl}_{3}\right)$

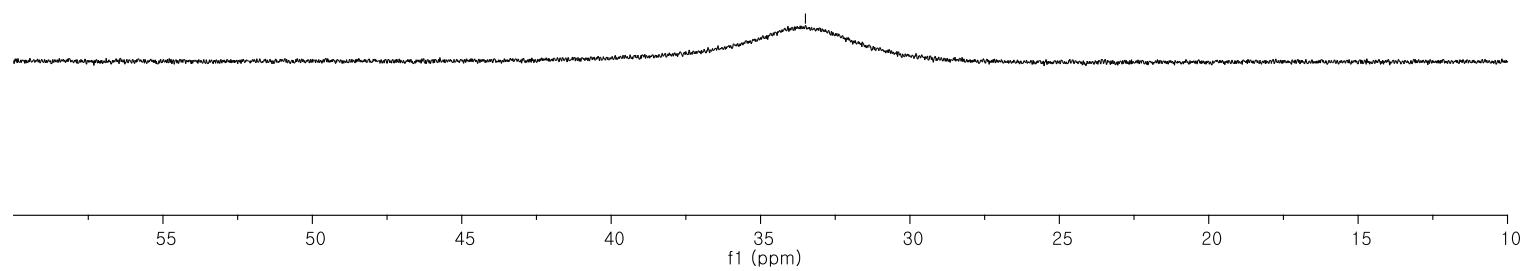


$N, N$-Dimethyl- $N^{\prime}-N$-[(1S,2R)-1-phenyl-2-(4,4,5,5-tetramethyl-1,3,2-dioxaborolan-2yl)propyl]sulfamide (Table $2,3 \mathrm{~m}$ )
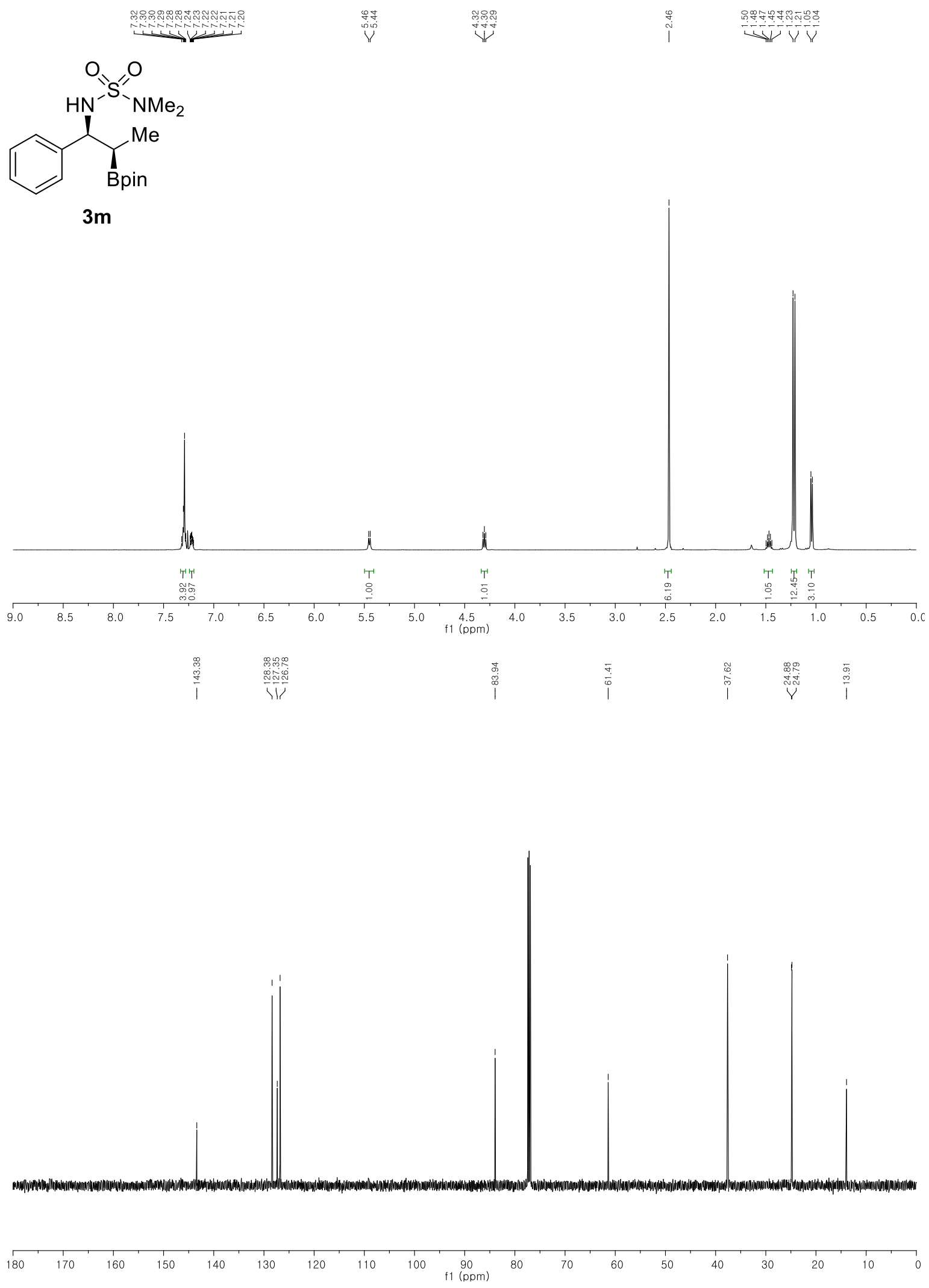
${ }^{11} \mathrm{~B}$ NMR (161 MHz, $\left.\mathrm{CDCl}_{3}\right)$

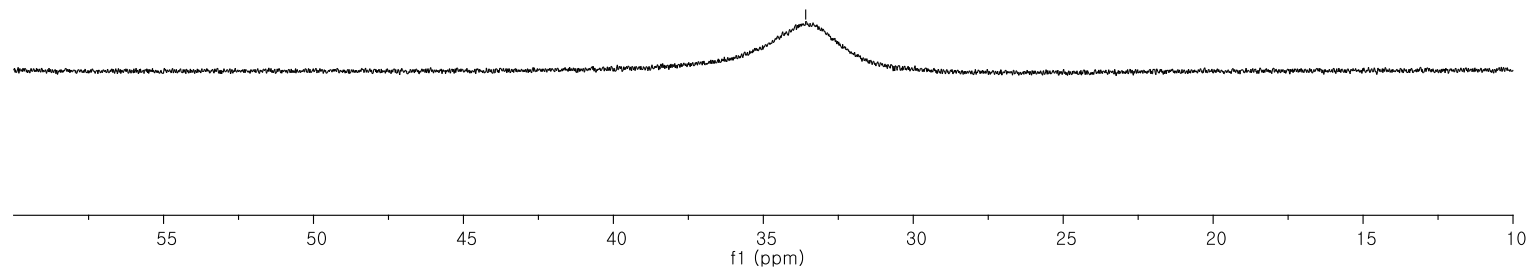


$N, N$-Dimethyl- $N^{\prime}-[(1 R, 2 R)$-2-hydroxy-1-phenylpropyl]sulfamide (4m)

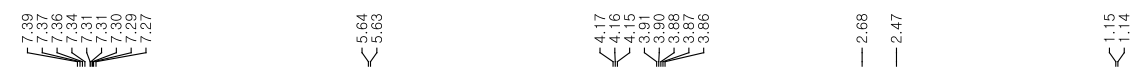<smiles>CC(O)[C@H](NS(C)(=O)=O)c1ccccc1</smiles>

$4 \mathrm{~m}$

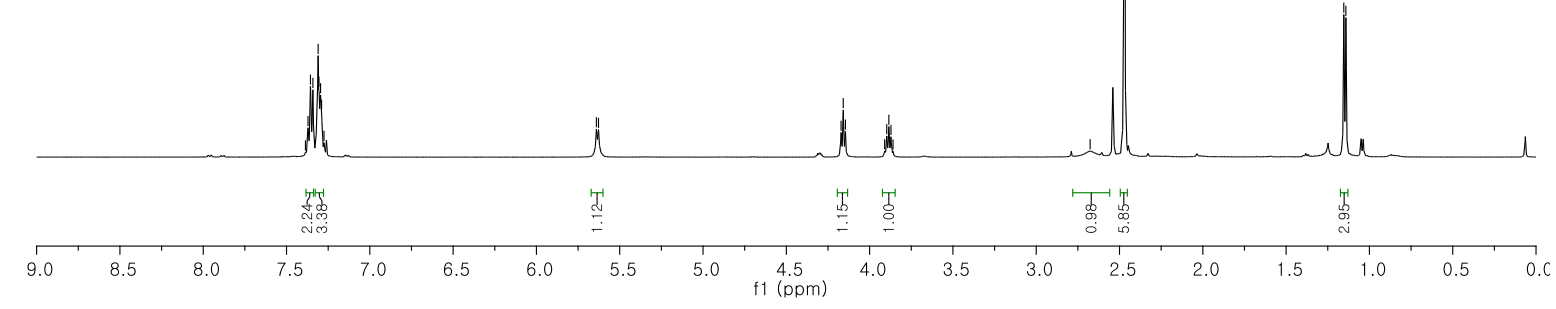

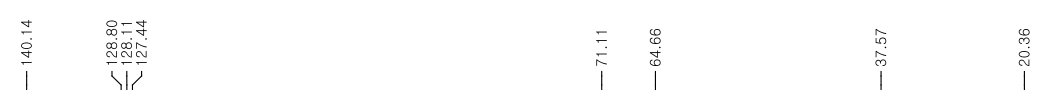

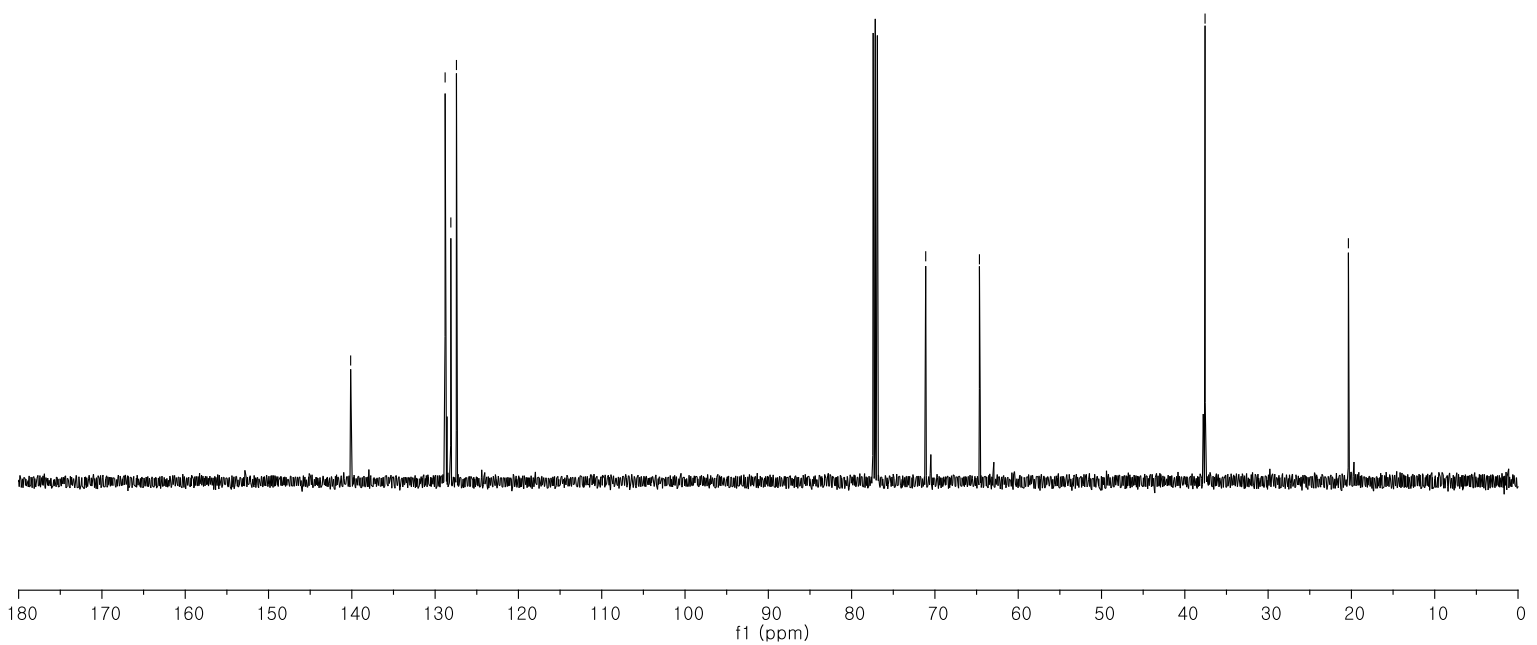


$N, N$-Dimethyl- $N^{\prime}$-[(1S,2R)-4-(1,3-dioxolan-2-yl)-1-phenyl-2-(4,4,5,5-tetramethyl-1,3,2dioxaborolan-2-yl)butyl]sulfamide (Table 2, 3n)

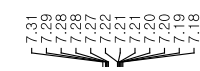

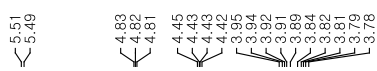

i<smiles>CS(=O)(=O)N[C@H](c1ccccc1)[C@@H](CCC1OCCO1)c1ccccc1</smiles>

$3 n$
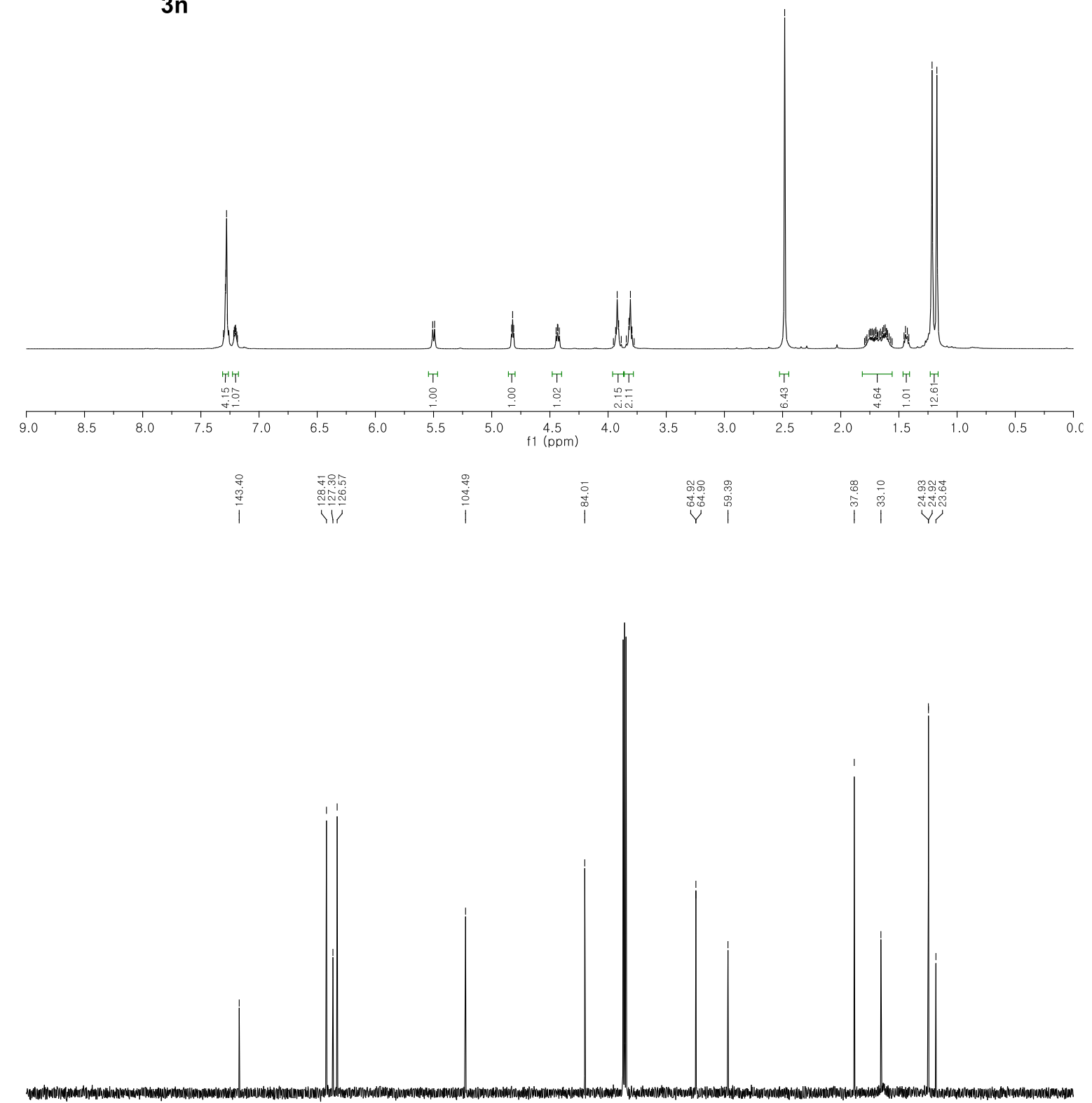

$180-170-160-150-140=130$

80

60

0 
${ }^{11} \mathrm{~B}$ NMR (161 MHz, $\left.\mathrm{CDCl}_{3}\right)$

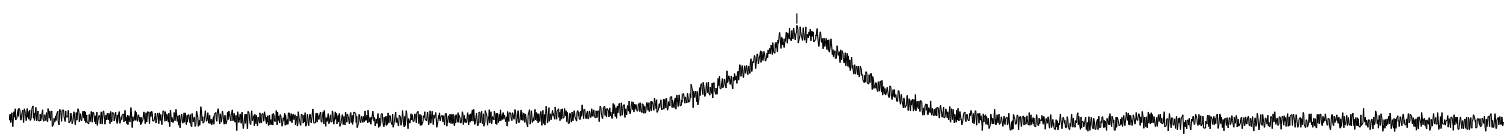

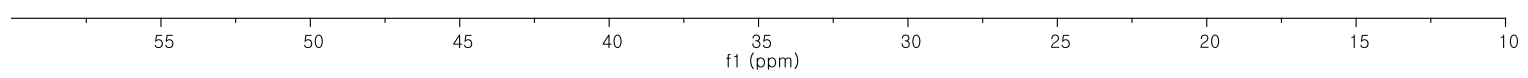


$N, N$-Dimethyl- $N^{\prime}-N^{\prime}$-[(1S,2R)-4-((tert-butyldimethylsilyl)oxy)-1-phenyl-2-(4,4,5,5tetramethyl-1,3,2-dioxaborolan-2-yl)butyl]sulfamide (Table 2, 3o)
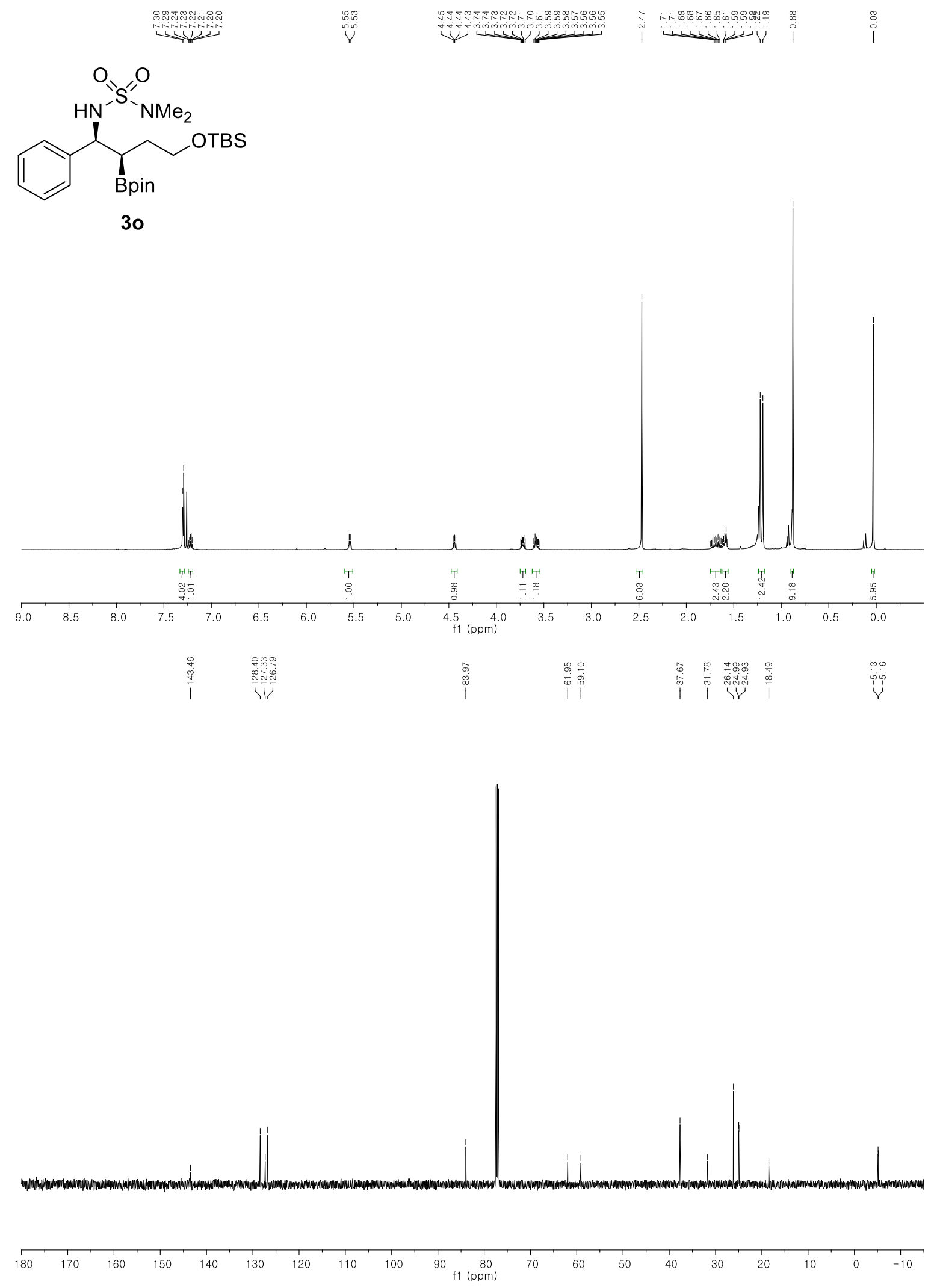
${ }^{11} \mathrm{~B}$ NMR (161 MHz, $\left.\mathrm{CDCl}_{3}\right)$

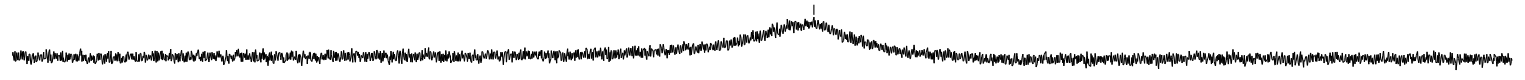

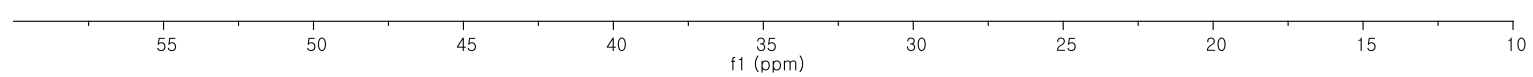


$N, N$-Dimethyl- $N^{\prime}-[(1 R, 2 R)-4-((t e r t-b u t y l d i m e t h y l s i l y l) o x y)-2-h y d r o x y-1-p h e n y l b u t y l]$ sulfamide (4o)
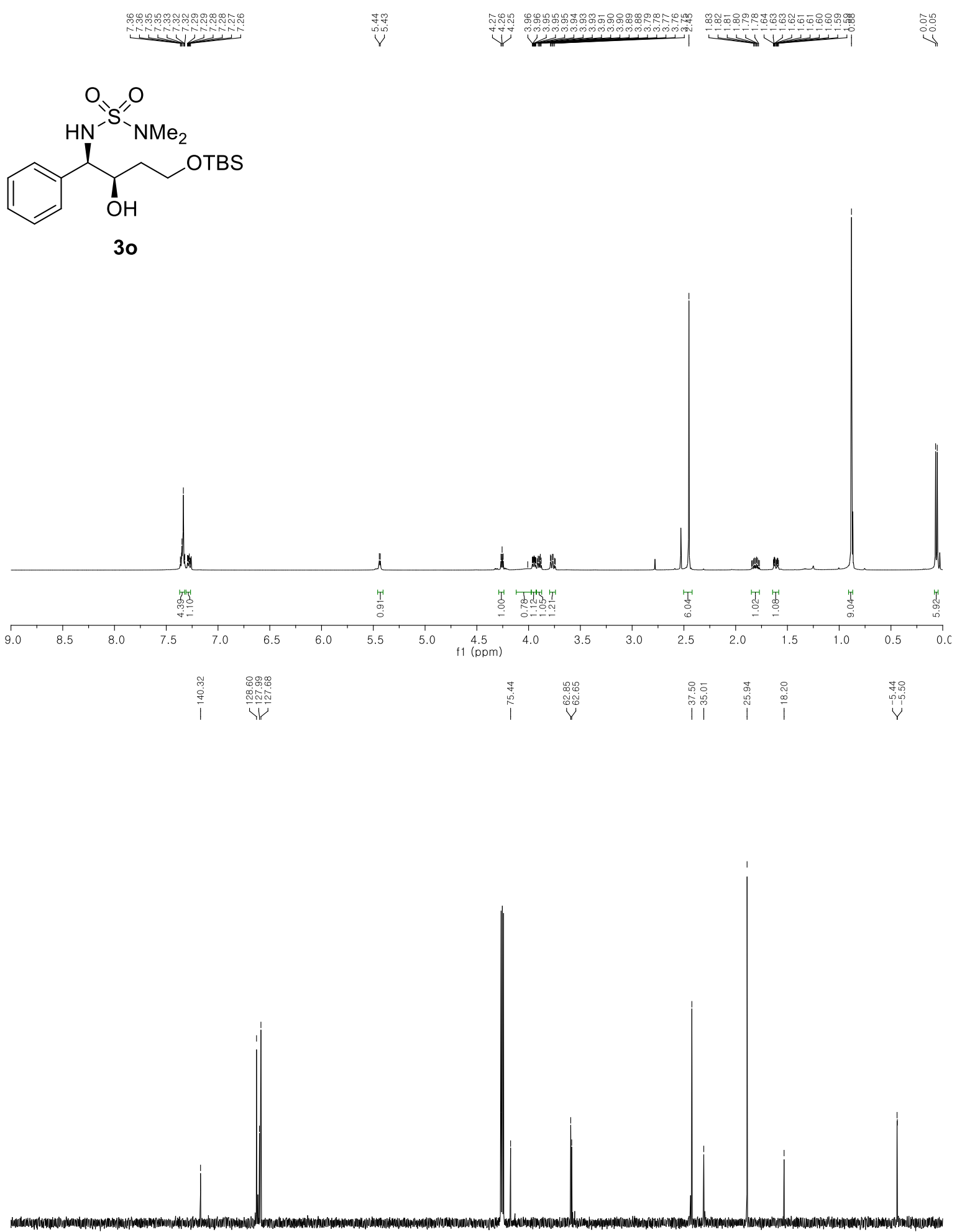

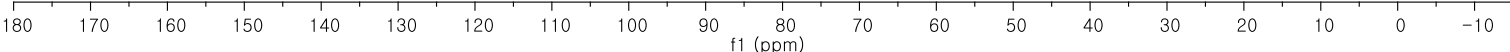


$N, N$-Dimethyl- $N$ '-((1S,2R)-1,4-diphenyl-2-(4,4,5,5-tetramethyl-1,3,2-dioxaborolan-2yl)butyl)- $N$ '-methylsulfamide ( $N$-methylated 3a)
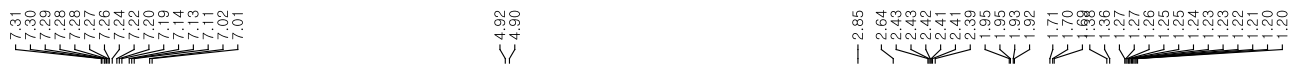<smiles>CN(C(c1ccccc1)[C@H](CCc1ccccc1)c1ccccc1)S(C)(=O)=O</smiles>

$N$-methylated 3a
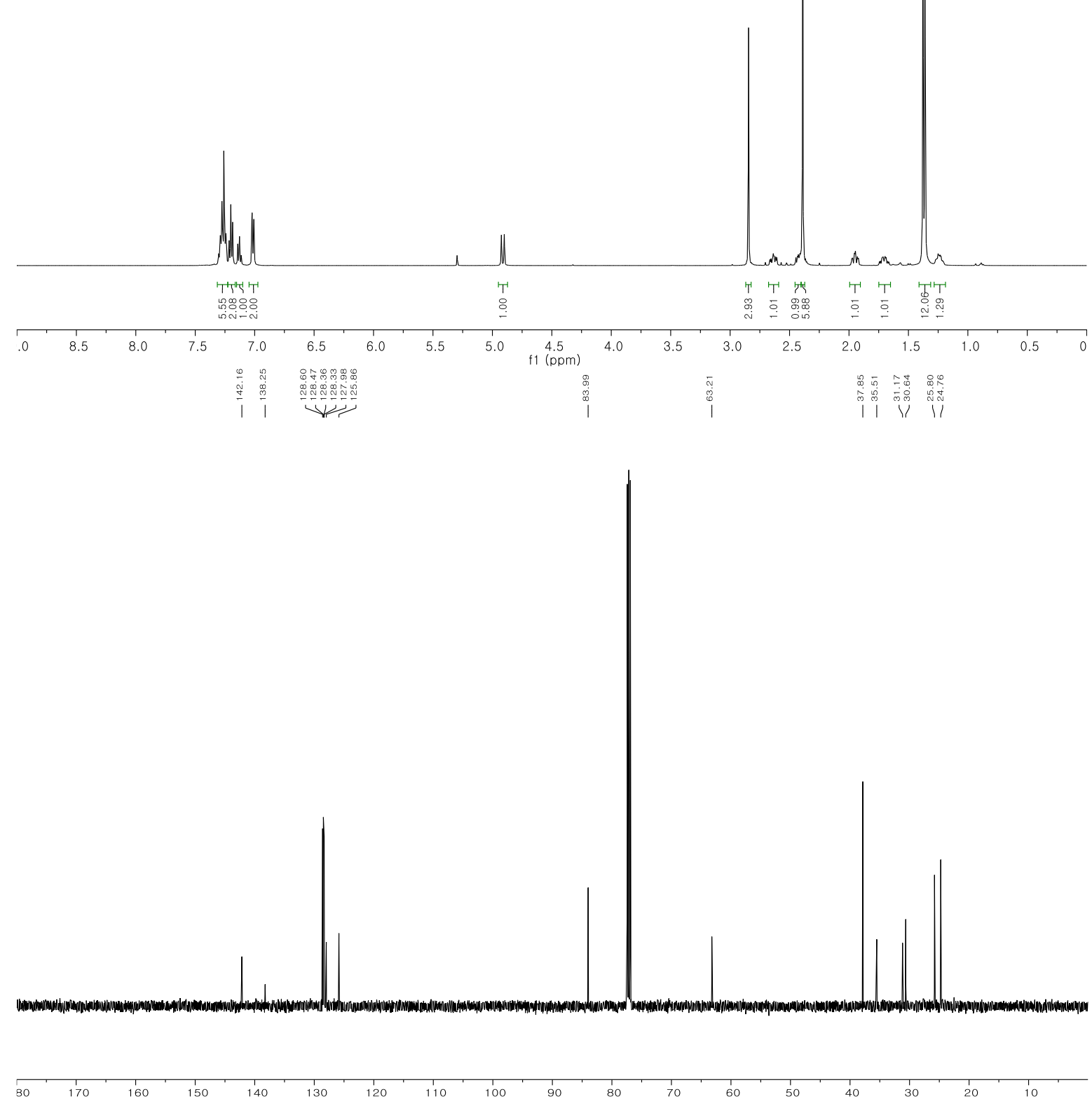

90

$80 \quad 70$

50 
${ }^{11} \mathrm{~B}$ NMR (161 MHz, $\left.\mathrm{CDCl}_{3}\right)$

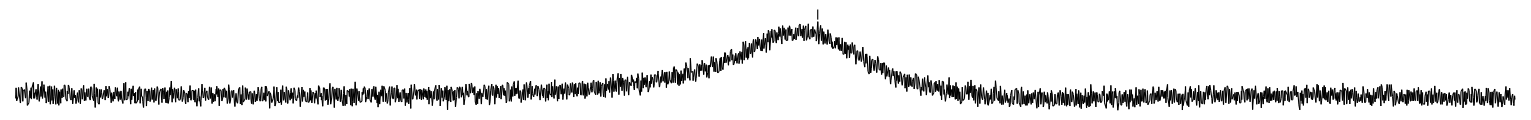

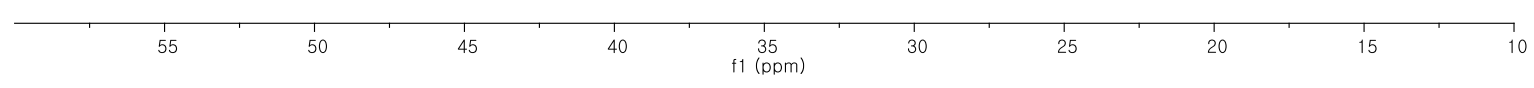


$N, N$-Dimethyl- $N$ '-[(1S,2S)-2-phenethyl-1-phenylbut-3-en-1-yl]sulfamide (Scheme 2, 4)

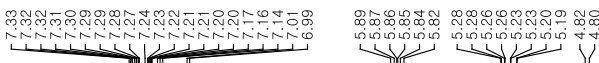<smiles>C=C[C@H](CCc1ccccc1)C(c1ccccc1)N(C)S(C)(=O)=O</smiles>

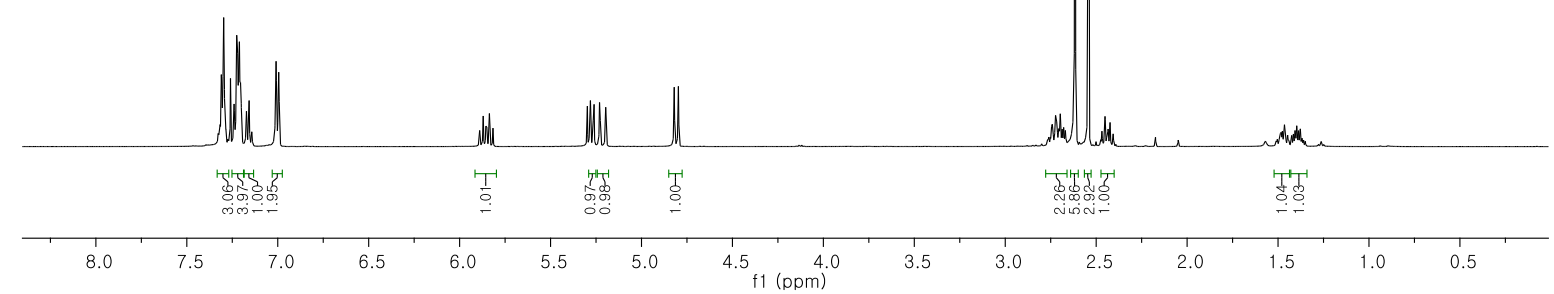

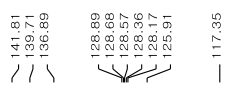

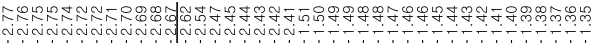
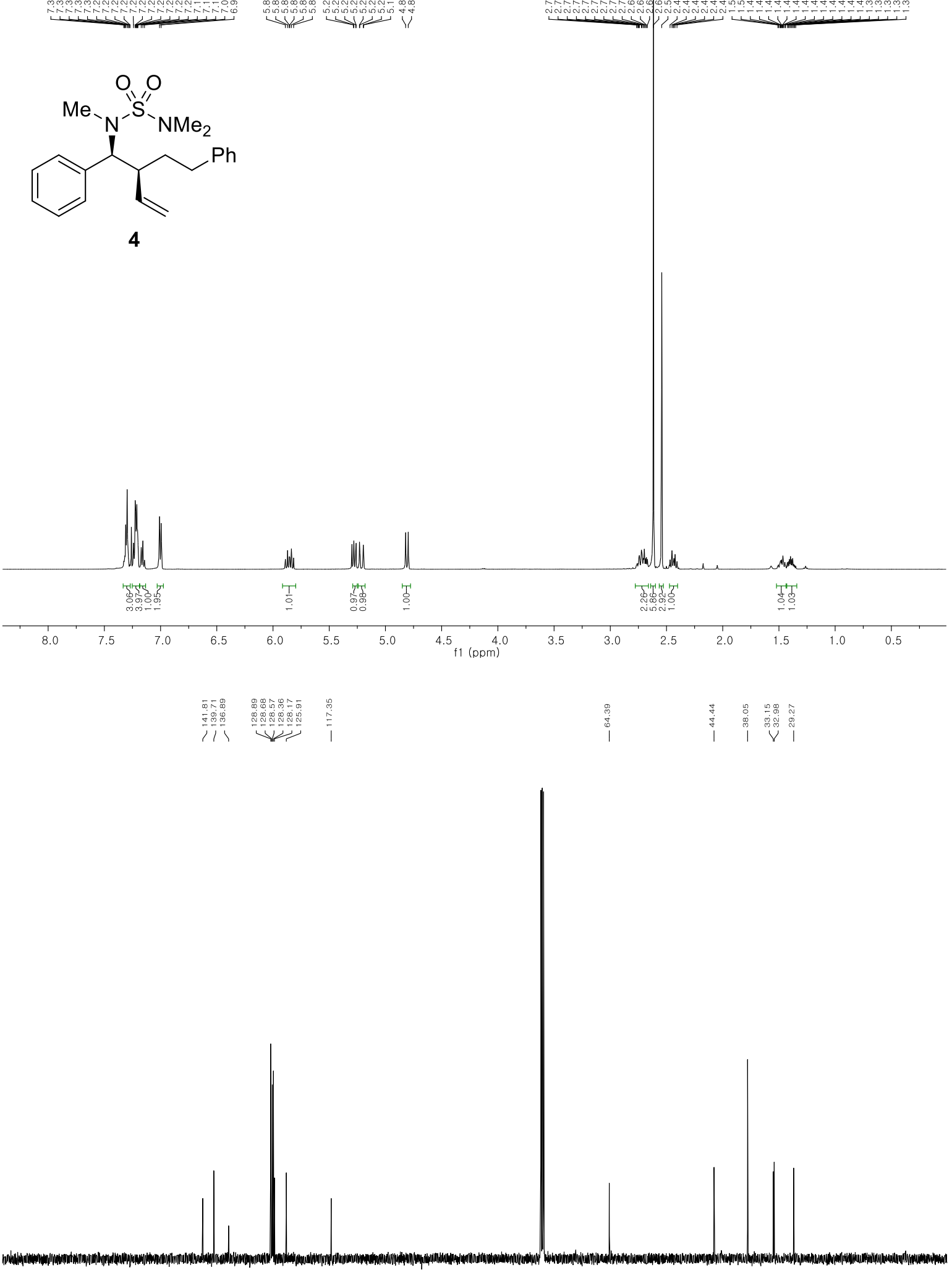
$N, N$-Dimethyl- $N$ '-[(1R,2R)-2-hydroxy-1,4-diphenylbuty]lsulfamide (Scheme 2, 5)<smiles>CNS(=O)(=O)NC(c1ccccc1)C(O)CCc1ccccc1</smiles>
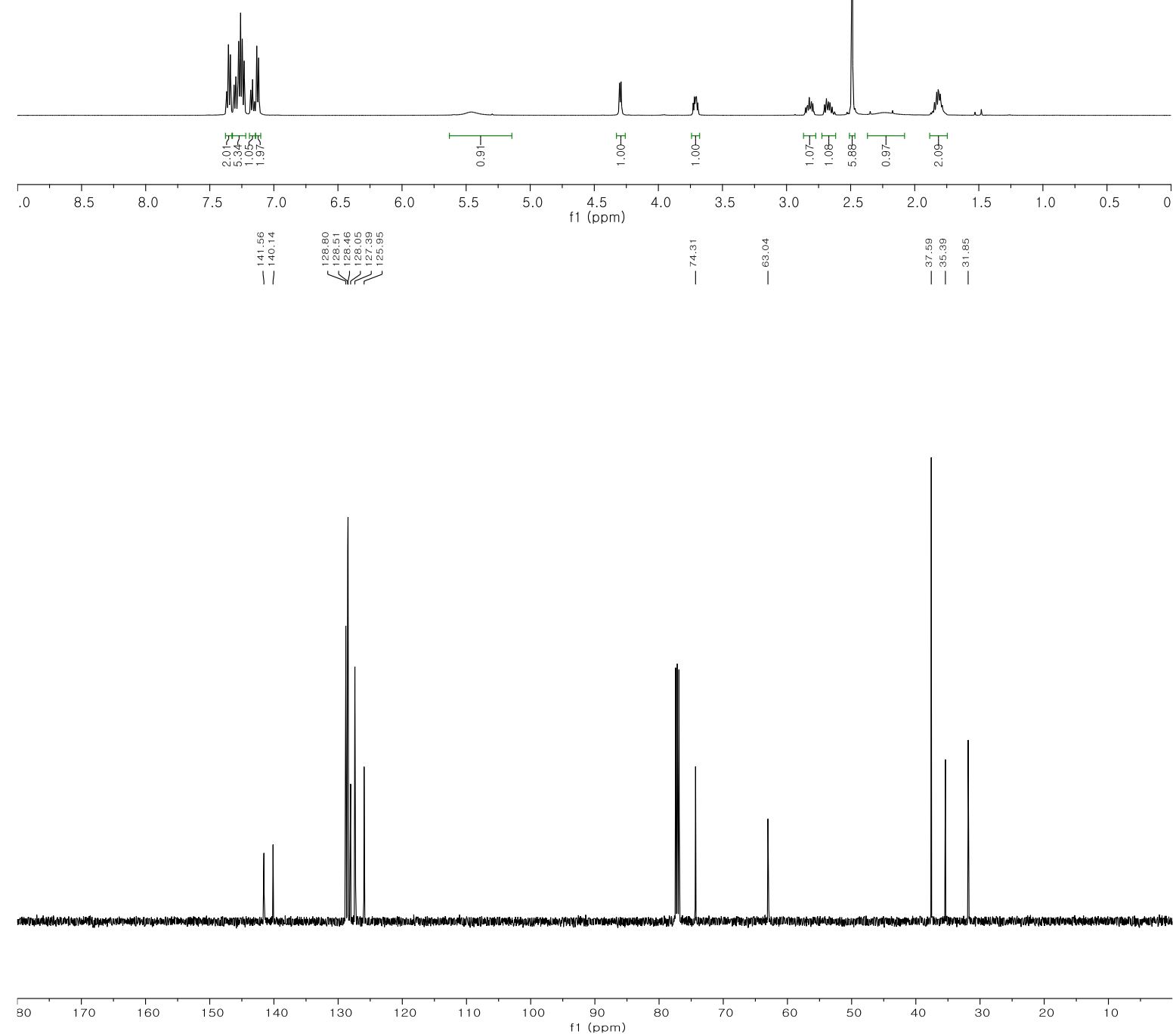
(2S,3R)-N,N-Dimethyl-2-phenethyl-3-phenylaziridine-1-sulfonamide (Scheme 2, 6)

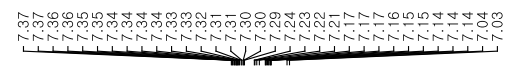

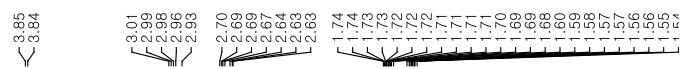<smiles>CS(=O)(=O)N(CCc1ccccc1)C(c1ccccc1)c1ccccc1</smiles>

6
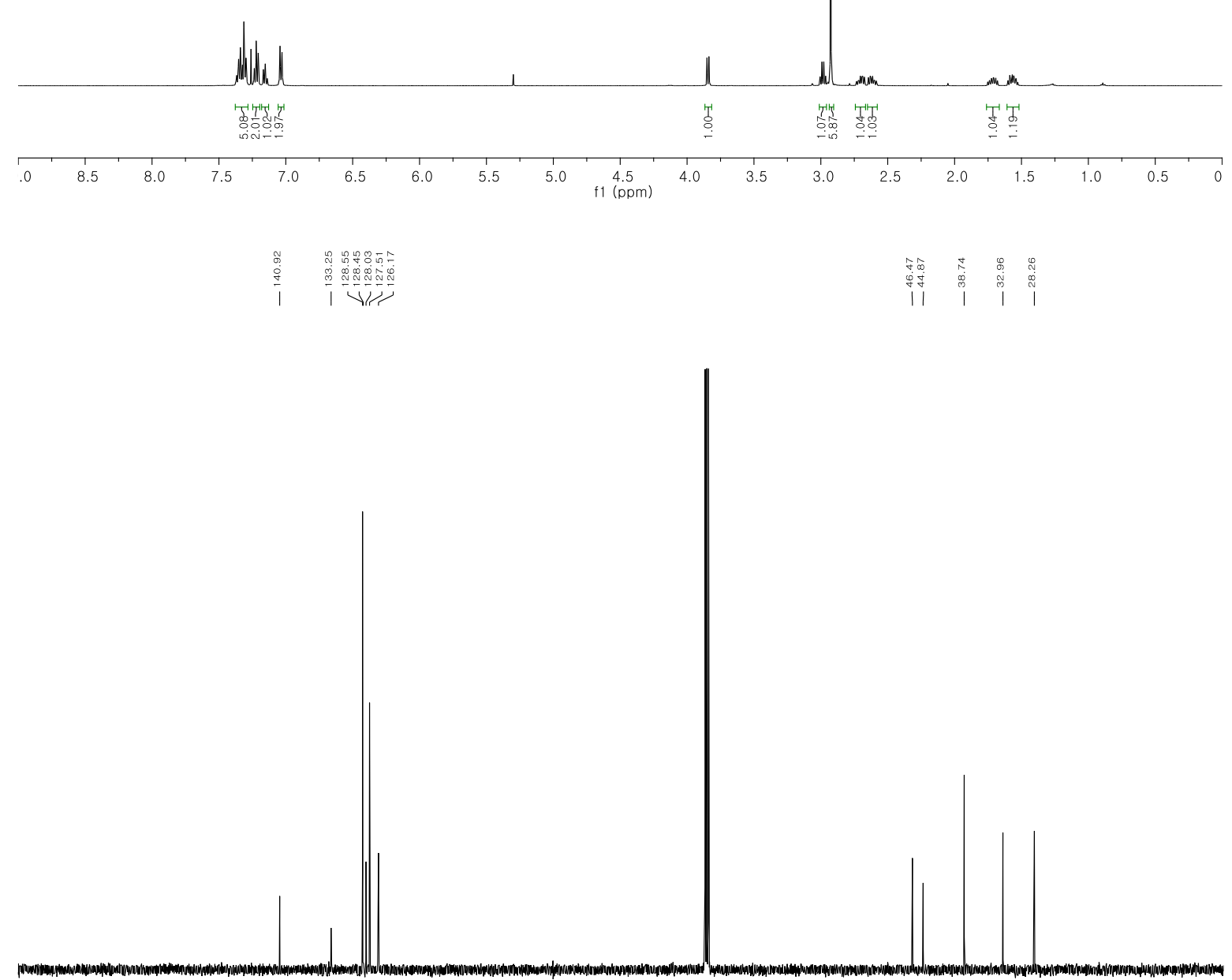

80 1
0

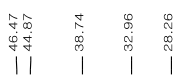




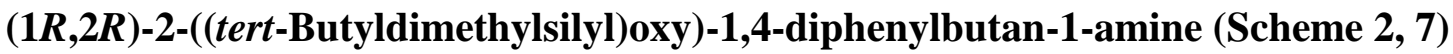

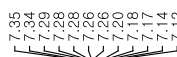

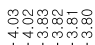

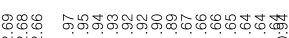<smiles>NC(c1ccccc1)C([O+])CCc1ccccc1</smiles>
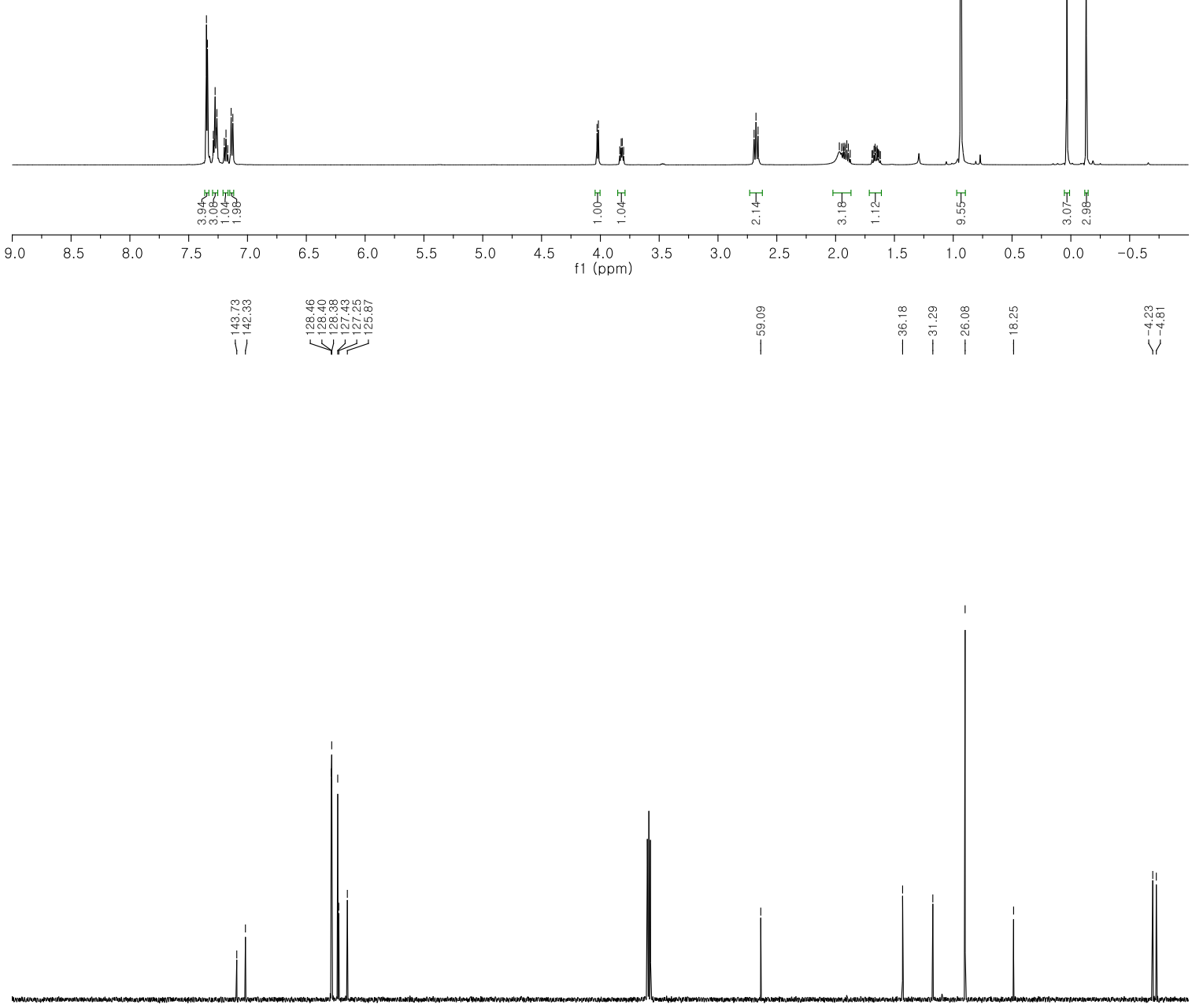

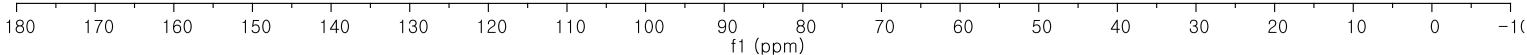


Appendix II

\section{Crystallographic Data of 3a}




\section{Crystallographic data of 3a}

CCDC 1831185 contains the supplementary crystallographic data for 3a. These data can be obtained free of charge via www.ccdc.cam.ac.uk/structures (or from the Cambridge Crystallographic Data Centre, 12, Union Road, Cambridge CD21EZ, UK; fax (+44) 1223336-033; or deposit@ccdc.cam.ac.uk). All the non-hydrogen atoms were refined anisotropically and hydrogen atoms were added to their ideal positions.

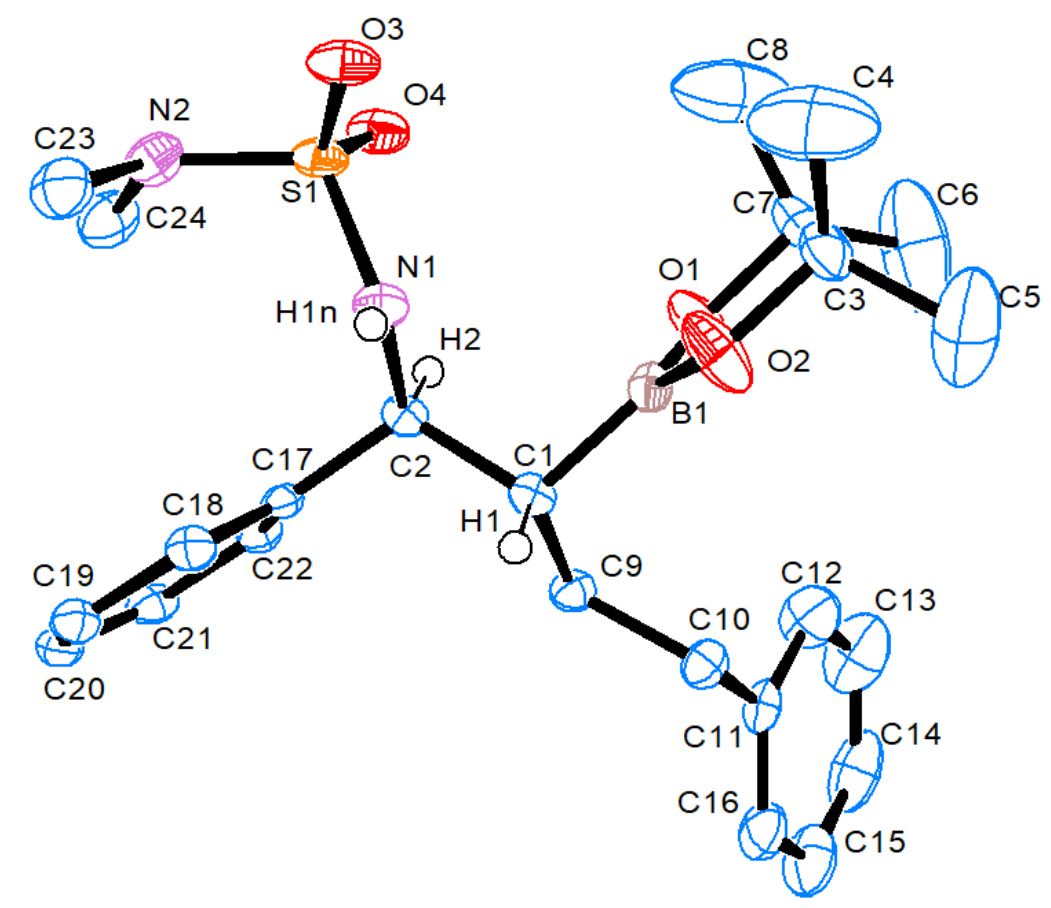

Figure S1. ORTEP diagram of $N, N$-Dimethyl- $N^{\prime}-[(1 S, 2 R)-1,4-$ diphenyl-2-(4,4,5,5tetramethyl-1,3,2-dioxaborolan-2-yl)butyl]sulfamide (3a), ellipsoids are shown at 50\% probability level. 
Table S2. Crystal data and structure refinement for $\mathbf{3 a}$.

Empirical formula

Formula weight

Temperature

Wavelength

Crystal system

Space group

Unit cell dimensions

Volume

$\mathrm{Z}$

Density (calculated)

Absorption coefficient

$\mathrm{F}(000)$

Crystal size

Theta range for data collection

Index ranges

Reflections collected

Independent reflections

Completeness to theta $=25.242^{\circ}$

Absorption correction

Max. and min. transmission

Refinement method

Data / restraints / parameters

Goodness-of-fit on $\mathrm{F}^{2}$

Final R indices [I>2sigma(I)]

$\mathrm{R}$ indices (all data)

Absolute structure parameter

Largest diff. peak and hole
$\mathrm{C}_{24} \mathrm{H}_{35} \mathrm{BN}_{2} \mathrm{O}_{4} \mathrm{~S}$

458.41

120(2) K

$0.71073 \AA$

Monoclinic

$P 21$

$\mathrm{a}=11.4365(8) \AA \quad \alpha=90^{\circ}$

$\mathrm{b}=7.5623(5) \AA$

$\beta=110.202(2)^{\circ}$

$\mathrm{c}=15.4088(11) \AA$

$\gamma=90^{\circ}$

$1250.66(15) \AA^{3}$

2

$1.217 \mathrm{Mg} / \mathrm{m}^{3}$

$0.161 \mathrm{~mm}^{-1}$

492

$0.450 \times 0.420 \times 0.160 \mathrm{~mm}^{3}$

3.040 to $28.379^{\circ}$.

$-14<=\mathrm{h}<=15,-10<=\mathrm{k}<=10,-20<=\mathrm{l}<=20$

26977

$6190[\mathrm{R}($ int $)=0.0405]$

$99.7 \%$

Semi-empirical from equivalents

0.7457 and 0.6838

Full-matrix least-squares on $\mathrm{F}^{2}$

6190 / 66 / 298

1.025

$\mathrm{R} 1=0.0468, \mathrm{wR} 2=0.1037$

$\mathrm{R} 1=0.0648, \mathrm{wR} 2=0.1130$

0.00(2) (from shelx: 0.004(23))

0.507 and $-0.418 \mathrm{e} \cdot \AA^{-3}$ 
Table S3. Atomic coordinates ( $\times 10^{4}$ ) and equivalent isotropic displacement parameters $\left(\AA^{2} \times 10^{3}\right.$ ) for 3a. U(eq) is defined as one third of the trace of the orthogonalized $U^{i j}$ tensor.

\begin{tabular}{|c|c|c|c|c|}
\hline & $\mathrm{x}$ & $\mathrm{y}$ & $\mathrm{z}$ & $\mathrm{U}(\mathrm{eq})$ \\
\hline $\mathrm{C}(1)$ & $6548(3)$ & $3817(4)$ & $3452(2)$ & $18(1)$ \\
\hline $\mathrm{B}(1)$ & $6914(3)$ & $4840(5)$ & $2693(2)$ & $21(1)$ \\
\hline $\mathrm{O}(1)$ & $6284(2)$ & $6307(3)$ & $2279(2)$ & $35(1)$ \\
\hline $\mathrm{O}(2)$ & $7832(3)$ & $4379(4)$ & $2369(2)$ & $45(1)$ \\
\hline$C(2)$ & $6898(3)$ & $5029(4)$ & $4311(2)$ & $18(1)$ \\
\hline$C(3)$ & $7842(3)$ & $5603(5)$ & $1652(2)$ & $32(1)$ \\
\hline$C(4)$ & $9144(4)$ & $6239(8)$ & $1868(5)$ & $85(2)$ \\
\hline$C(5)$ & $7456(6)$ & 4495(9) & $778(3)$ & $87(2)$ \\
\hline$C(6)$ & $5862(5)$ & $7341(12)$ & $752(3)$ & $99(2)$ \\
\hline$C(7)$ & $6863(3)$ & $7033(5)$ & $1655(2)$ & $32(1)$ \\
\hline $\mathrm{C}(8)$ & $7464(6)$ & $8744(7)$ & $2119(5)$ & $85(2)$ \\
\hline $\mathrm{C}(9)$ & $5140(3)$ & $3425(4)$ & $3086(2)$ & $20(1)$ \\
\hline$C(10)$ & $4716(3)$ & $2353(5)$ & $2181(2)$ & $23(1)$ \\
\hline $\mathrm{C}(11)$ & $3350(3)$ & $2582(5)$ & $1642(2)$ & $25(1)$ \\
\hline$C(12)$ & 2922(4) & $4188(6)$ & $1205(3)$ & $43(1)$ \\
\hline$C(13)$ & $1662(4)$ & $4435(7)$ & $712(3)$ & $58(1)$ \\
\hline$C(14)$ & $819(4)$ & $3116(7)$ & $658(3)$ & $52(1)$ \\
\hline$C(15)$ & $1220(4)$ & $1540(7)$ & 1093(3) & $49(1)$ \\
\hline$C(16)$ & $2486(3)$ & $1270(5)$ & $1580(2)$ & $36(1)$ \\
\hline$C(17)$ & $6620(3)$ & $4248(4)$ & $5130(2)$ & $17(1)$ \\
\hline $\mathrm{C}(18)$ & $7422(3)$ & $3072(4)$ & $5744(2)$ & $21(1)$ \\
\hline$C(19)$ & $7158(3)$ & $2465(5)$ & $6511(2)$ & $25(1)$ \\
\hline $\mathrm{C}(20)$ & $6071(3)$ & 2994(4) & $6650(2)$ & $25(1)$ \\
\hline $\mathrm{C}(21)$ & $5247(3)$ & 4107(4) & $6025(2)$ & $23(1)$ \\
\hline$C(22)$ & $5523(3)$ & $4753(4)$ & $5269(2)$ & $20(1)$ \\
\hline$S(1)$ & $8754(1)$ & $7475(1)$ & $4947(1)$ & $23(1)$ \\
\hline $\mathrm{O}(3)$ & $9946(2)$ & $7585(4)$ & $4833(2)$ & $34(1)$ \\
\hline $\mathrm{O}(4)$ & $7810(2)$ & $8748(3)$ & $4524(2)$ & $30(1)$ \\
\hline $\mathrm{N}(1)$ & $8213(2)$ & $5555(4)$ & $4527(2)$ & $22(1)$ \\
\hline $\mathrm{N}(2)$ & $9043(2)$ & $7609(4)$ & $6059(2)$ & $29(1)$ \\
\hline$C(23)$ & 9993(3) & $6378(5)$ & $6633(3)$ & $36(1)$ \\
\hline$C(24)$ & $7996(3)$ & $7892(5)$ & $6386(2)$ & $36(1)$ \\
\hline
\end{tabular}


Table S4. Bond lengths $[\AA]$ and angles $\left[^{\circ}\right]$ for $\mathbf{3 a}$.

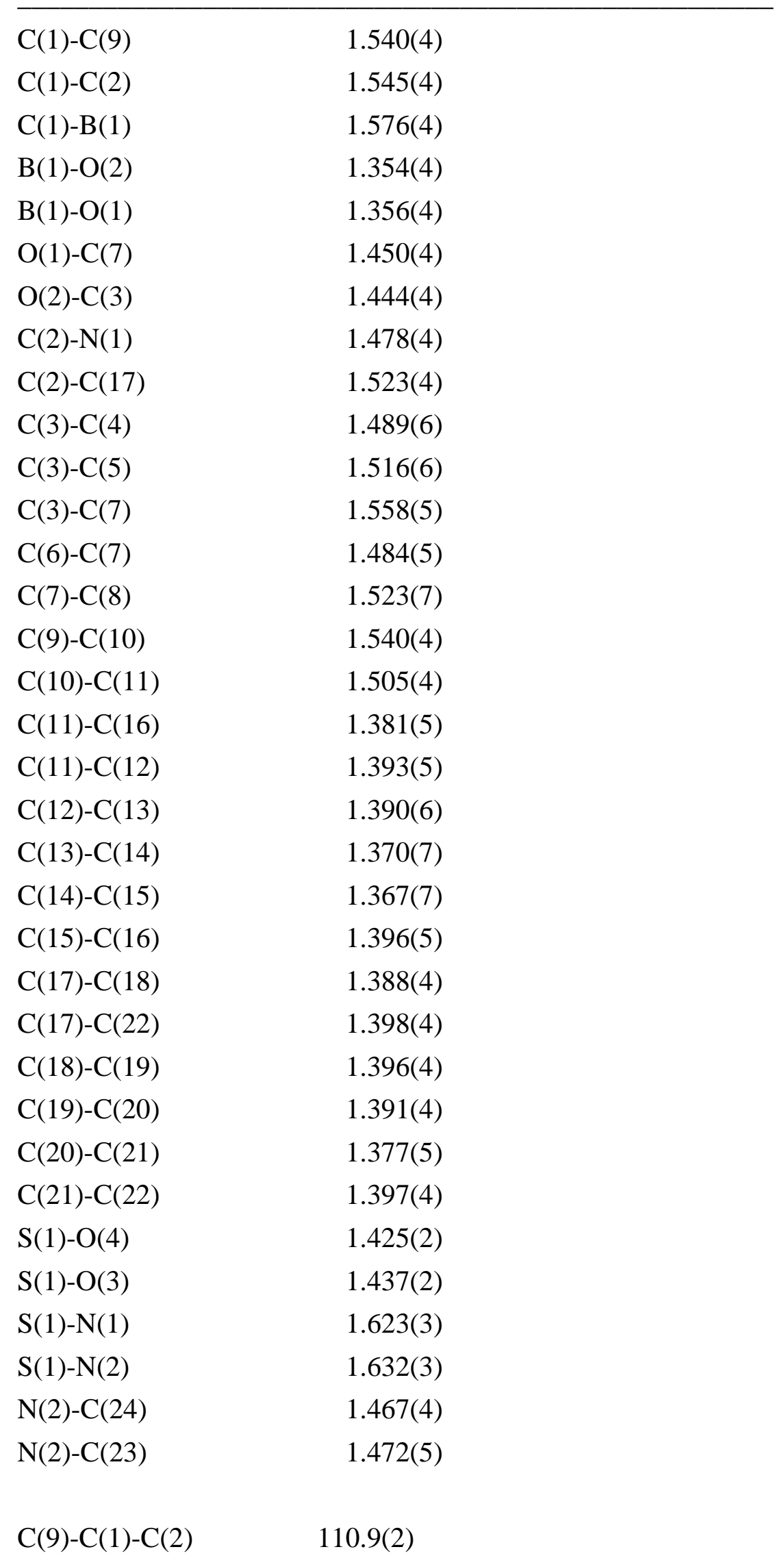




$\begin{array}{ll}\mathrm{C}(9)-\mathrm{C}(1)-\mathrm{B}(1) & 109.6(2) \\ \mathrm{C}(2)-\mathrm{C}(1)-\mathrm{B}(1) & 106.7(2) \\ \mathrm{O}(2)-\mathrm{B}(1)-\mathrm{O}(1) & 113.0(3) \\ \mathrm{O}(2)-\mathrm{B}(1)-\mathrm{C}(1) & 125.9(3) \\ \mathrm{O}(1)-\mathrm{B}(1)-\mathrm{C}(1) & 121.1(3) \\ \mathrm{B}(1)-\mathrm{O}(1)-\mathrm{C}(7) & 109.3(3) \\ \mathrm{B}(1)-\mathrm{O}(2)-\mathrm{C}(3) & 109.2(3) \\ \mathrm{N}(1)-\mathrm{C}(2)-\mathrm{C}(17) & 113.9(2) \\ \mathrm{N}(1)-\mathrm{C}(2)-\mathrm{C}(1) & 107.2(2) \\ \mathrm{C}(17)-\mathrm{C}(2)-\mathrm{C}(1) & 114.4(2) \\ \mathrm{O}(2)-\mathrm{C}(3)-\mathrm{C}(4) & 107.9(4) \\ \mathrm{O}(2)-\mathrm{C}(3)-\mathrm{C}(5) & 104.4(4) \\ \mathrm{C}(4)-\mathrm{C}(3)-\mathrm{C}(5) & 109.8(5) \\ \mathrm{O}(2)-\mathrm{C}(3)-\mathrm{C}(7) & 104.3(2) \\ \mathrm{C}(4)-\mathrm{C}(3)-\mathrm{C}(7) & 116.1(4) \\ \mathrm{C}(5)-\mathrm{C}(3)-\mathrm{C}(7) & 113.4(3) \\ \mathrm{O}(1)-\mathrm{C}(7)-\mathrm{C}(6) & 107.5(4) \\ \mathrm{O}(1)-\mathrm{C}(7)-\mathrm{C}(8) & 104.2(3) \\ \mathrm{C}(6)-\mathrm{C}(7)-\mathrm{C}(8) & 112.5(5) \\ \mathrm{O}(1)-\mathrm{C}(7)-\mathrm{C}(3) & 103.5(2) \\ \mathrm{C}(6)-\mathrm{C}(7)-\mathrm{C}(3) & 115.3(4) \\ \mathrm{C}(8)-\mathrm{C}(7)-\mathrm{C}(3) & 112.5(3) \\ \mathrm{C}(1)-\mathrm{C}(9)-\mathrm{C}(10) & 113.3(2) \\ \mathrm{C}(11)-\mathrm{C}(10)-\mathrm{C}(9) & 112.4(2) \\ \mathrm{C}(16)-\mathrm{C}(11)-\mathrm{C}(12) & 118.0(3) \\ \mathrm{C}(16)-\mathrm{C}(11)-\mathrm{C}(10) & 122.5(3) \\ \mathrm{C}(12)-\mathrm{C}(11)-\mathrm{C}(10) & 119.5(3) \\ \mathrm{C}(13)-\mathrm{C}(12)-\mathrm{C}(11) & 120.5(4) \\ \mathrm{C}(14)-\mathrm{C}(13)-\mathrm{C}(12) & 120.7(4) \\ \mathrm{C}(15)-\mathrm{C}(14)-\mathrm{C}(13) & 119.7(4) \\ \mathrm{C}(14)-\mathrm{C}(15)-\mathrm{C}(16) & 120.1(4) \\ \mathrm{C}(11)-\mathrm{C}(16)-\mathrm{C}(15) & 121.1(4) \\ \mathrm{C}(18)-\mathrm{C}(17)-\mathrm{C}(22) & 119.2(3) \\ \mathrm{C}(18)-\mathrm{C}(17)-\mathrm{C}(2) & 122.4(2) \\ & 118.5(2) \\ \mathrm{C}(2)-\mathrm{C}(19) & 120.2(3) \\ & \end{array}$




$\begin{array}{ll}\mathrm{C}(20)-\mathrm{C}(21)-\mathrm{C}(22) & 120.0(3) \\ \mathrm{C}(21)-\mathrm{C}(22)-\mathrm{C}(17) & 120.4(3) \\ \mathrm{O}(4)-\mathrm{S}(1)-\mathrm{O}(3) & 120.41(15) \\ \mathrm{O}(4)-\mathrm{S}(1)-\mathrm{N}(1) & 107.33(14) \\ \mathrm{O}(3)-\mathrm{S}(1)-\mathrm{N}(1) & 104.47(15) \\ \mathrm{O}(4)-\mathrm{S}(1)-\mathrm{N}(2) & 106.59(15) \\ \mathrm{O}(3)-\mathrm{S}(1)-\mathrm{N}(2) & 105.54(13) \\ \mathrm{N}(1)-\mathrm{S}(1)-\mathrm{N}(2) & 112.66(15) \\ \mathrm{C}(2)-\mathrm{N}(1)-\mathrm{S}(1) & 123.3(2) \\ \mathrm{C}(24)-\mathrm{N}(2)-\mathrm{C}(23) & 114.0(3) \\ \mathrm{C}(24)-\mathrm{N}(2)-\mathrm{S}(1) & 118.5(2) \\ \mathrm{C}(23)-\mathrm{N}(2)-\mathrm{S}(1) & 115.6(2)\end{array}$

Symmetry transformations used to generate equivalent atoms: 
Table S5. Anisotropic displacement parameters $\left(\AA^{2} \times 10^{3}\right)$ for 3a. The anisotropic displacement factor exponent takes the form: $-2 \pi^{2}\left[h^{2} a^{* 2} U^{11}+\ldots+2 h k a^{*} b^{*} U^{12}\right]$

\begin{tabular}{|c|c|c|c|c|c|c|}
\hline & $\mathrm{U}^{11}$ & $\mathrm{U}^{22}$ & $\mathrm{U}^{33}$ & $\mathrm{U}^{23}$ & $\mathrm{U}^{13}$ & $\mathrm{U}^{12}$ \\
\hline $\mathrm{C}(1)$ & $15(1)$ & $19(1)$ & $22(1)$ & 1(1) & $9(1)$ & $2(1)$ \\
\hline $\mathrm{B}(1)$ & $17(2)$ & $28(2)$ & $19(2)$ & $-2(1)$ & $6(1)$ & $-1(1)$ \\
\hline $\mathrm{O}(1)$ & $29(1)$ & $44(2)$ & $40(1)$ & $20(1)$ & $22(1)$ & $12(1)$ \\
\hline $\mathrm{O}(2)$ & $52(2)$ & $50(2)$ & $50(2)$ & $21(1)$ & $41(1)$ & $22(1)$ \\
\hline$C(2)$ & $11(1)$ & $21(1)$ & $22(1)$ & $2(1)$ & $8(1)$ & 1(1) \\
\hline$C(3)$ & $30(2)$ & $43(2)$ & $29(2)$ & $4(2)$ & $17(1)$ & $-3(2)$ \\
\hline C(4) & $36(3)$ & $68(3)$ & $146(5)$ & $19(4)$ & $24(3)$ & $-3(2)$ \\
\hline$C(5)$ & $116(5)$ & $95(4)$ & $44(3)$ & $-11(3)$ & $21(3)$ & $28(4)$ \\
\hline$C(6)$ & $70(3)$ & $177(6)$ & $48(2)$ & $36(4)$ & $17(2)$ & $66(4)$ \\
\hline$C(7)$ & $28(2)$ & $44(2)$ & $29(2)$ & $15(1)$ & $14(1)$ & $1(1)$ \\
\hline$C(8)$ & $79(4)$ & $46(3)$ & $161(6)$ & $-13(3)$ & $83(4)$ & $-11(3)$ \\
\hline $\mathrm{C}(9)$ & $18(1)$ & $21(2)$ & $23(1)$ & $-2(1)$ & $10(1)$ & $-2(1)$ \\
\hline$C(10)$ & $21(1)$ & $24(2)$ & $24(1)$ & $-4(1)$ & $9(1)$ & 1(1) \\
\hline $\mathrm{C}(11)$ & $26(1)$ & $32(2)$ & $16(1)$ & $-6(1)$ & $7(1)$ & $1(2)$ \\
\hline$C(12)$ & $41(2)$ & $39(2)$ & $41(2)$ & $1(2)$ & $4(2)$ & $6(2)$ \\
\hline$C(13)$ & $53(3)$ & $55(3)$ & $49(2)$ & $-3(2)$ & $-5(2)$ & $26(2)$ \\
\hline$C(14)$ & $29(2)$ & $81(3)$ & $37(2)$ & $-19(2)$ & $1(2)$ & $12(2)$ \\
\hline$C(15)$ & $30(2)$ & $78(3)$ & $34(2)$ & $-11(2)$ & $6(2)$ & $-14(2)$ \\
\hline$C(16)$ & $32(2)$ & $46(2)$ & $24(2)$ & $-4(2)$ & $2(1)$ & $-8(2)$ \\
\hline$C(17)$ & $16(1)$ & $16(1)$ & $18(1)$ & $-3(1)$ & $6(1)$ & $-5(1)$ \\
\hline$C(18)$ & $16(1)$ & $20(1)$ & $25(1)$ & $0(1)$ & $6(1)$ & 1(1) \\
\hline$C(19)$ & $28(1)$ & $20(1)$ & $24(1)$ & $1(1)$ & $5(1)$ & $-2(2)$ \\
\hline$C(20)$ & $34(2)$ & $22(2)$ & $23(1)$ & $-3(1)$ & $14(1)$ & $-9(1)$ \\
\hline $\mathrm{C}(21)$ & $20(2)$ & $24(2)$ & $30(2)$ & $-7(1)$ & $16(1)$ & $-7(1)$ \\
\hline$C(22)$ & $16(1)$ & $20(1)$ & $23(1)$ & $-3(1)$ & $7(1)$ & $-2(1)$ \\
\hline$S(1)$ & $14(1)$ & $22(1)$ & $32(1)$ & $2(1)$ & $8(1)$ & $-4(1)$ \\
\hline $\mathrm{O}(3)$ & $18(1)$ & $36(1)$ & $50(1)$ & $0(1)$ & $16(1)$ & $-10(1)$ \\
\hline $\mathrm{O}(4)$ & $24(1)$ & $22(1)$ & $41(1)$ & $6(1)$ & $7(1)$ & $-1(1)$ \\
\hline $\mathrm{N}(1)$ & $14(1)$ & $24(1)$ & $30(1)$ & $0(1)$ & $11(1)$ & $-1(1)$ \\
\hline $\mathrm{N}(2)$ & $23(1)$ & $30(1)$ & $33(1)$ & $-5(1)$ & $6(1)$ & $-1(1)$ \\
\hline$C(23)$ & $29(2)$ & $34(2)$ & $37(2)$ & $3(2)$ & 1(1) & $1(2)$ \\
\hline$C(24)$ & $36(2)$ & $36(2)$ & $38(2)$ & $-12(2)$ & $16(2)$ & $-3(2)$ \\
\hline
\end{tabular}


Table S6. Hydrogen bonds for $\mathbf{3 a}\left[\AA\right.$ and $\left.{ }^{\circ}\right]$.

\begin{tabular}{lllll}
\hline D-H...A & $d(D-H)$ & $d(H . . A)$ & $d(D \ldots A)$ & $<(D H A)$ \\
\hline $\mathrm{N}(1)-\mathrm{H}(1 \mathrm{~N}) \ldots \mathrm{O}(3) \# 1$ & $0.78(4)$ & $2.24(4)$ & $3.003(4)$ & $166(4)$ \\
\hline
\end{tabular}

Symmetry transformations used to generate equivalent atoms:

\#1 - $\mathrm{x}+2, \mathrm{y}-1 / 2,-\mathrm{z}+1$ 